\title{
XXVI. Ueber eine krystallochemische Theorie der Silicate.
}

Von

F. J. Wiik in Helsingfors.

(Hierzu Tafel V und 16 Textfiguren.)

In einer Abhandlung (Utkast till ett kristallokemiskt mineralsystem, I. Silikaterna; Acta Soc. Sc. Fenn. 19, No. 14, 221 pagg. 40) habe ich eine Theorie uber das Verhältniss zwischen den chemischen und krystallographischen Eigenschaften der Silicate dargestellt. Da diese Theorie, wie es mir scheint, einige streitige Fragen der mineralogischen Wissenschaft, unter anderen die optischen Anomalien, erklärt und weiter verfolgt zu werden verdient, gebe ich in dem Folgenden einen Auszug der obengenannten Abhandlung, und zwar zuerst des krystallographischen Theiles, darnach des chemischen und zuletzt die Darlegung der eigentlichen krystallochemischen 'Theorie.

\section{I.}

In dieser Zeitschr. 8, 205 habe ich einige Varietäten des Anorthites von finnischen Fundorten angeführt, welche sich durch einen eigenthúmlichen hemimorphen Habitus auszeichnen. Da indessen eine Hemimorphie in dem triklinen Systeme nicht vorkommen kann, aber wohl eine Hemiëdrie (die 32. Abtheilung der von A. Gadolin aufgestellten Eintheilung der Krystallsysteme, Acta Soc. Sc. Fenn. 9), und da jene Anorthitkrystalle vollkommen den Charakter dieser Abtheilung zeigen, indem sie Flächen ohne Gegenflächen, also kein Centrum der Symmetrie haben, so bin ich jetzt uberzeugt, dass in der That Anorthit hemièdrisch-triklinisch krystallisirt, obgleich er gewöhnlich scheinbar holoëdrisch auftritt, wie es ja mit mehreren anderen hemiëdrischen und tetartoëdrischen Mineralien der Fall ist. Die Ursache, dass die genannten Anorthite, besonders der sogenannle Lindsay it von Orijärvi und der Anorthoit von Sillböle, die hemiëdrische Ausbildung zeigen, ist wahrscheinlich darin zu suchen, dass sie in einge- 
wachsenen und vollständig isolirten Krystallen vorkommen, während dagegen der Anorthit von den meisten ubrigen Fundorlen in aufgewachsenen Krystallen auftritt und übrigens selten deutlich ausgebildet ist. Dasselbe Verhälniss zeigt auch der Skapolith, dessen eingewachsene Krystalle von Pargas die pyramidale Hemiëdrie zeigen, während die aufgewachsenen Krystalle von Laurinkaari scheinbar holoëdrisch sind. Die Lindsayitkrystalle sind gewöhnlich nach der Hauptaxe verlängert (1. c. Fig. 2 und 3), während die Anorthoitkrystalle, von welchen ich bisher nur zwei, aber ganz ähnliche Exemplare gefunden habe, nach der Brachydiagonale ( $a$-Axe) ausgezogen (l. c. Fig. 1), aber nur am einen Ende deutlich ausgebildet sind.

In Fig. 1 und 2 der beigefugten Tafel $\mathrm{V}$ habe ich diese zwei hemiëdrischen Anorthitformen (Fig. 1 Lindsayittypus, Fig. 2 Anorthoittypus), mit den Hauptläachen: $(110),(\bar{T} 10),(0 \bar{T} 0),(010),(001),(00 T)$ so gezeichnet, dass die relative Länge der Kanten dem Axenverhältnisse des Anorthites entspricht, und dass die Anortboitform (Fig. 2) durch (021) resp. $(0 \overline{2} 1)$ in kleinere hemiëdrische Partialformen getheilt wird. Wenn man nun auf die zwei Primitivformen des Anorthites die gewöhnlichen Zwillingsgesetze des Plagioklases anwendet, nämlich für Typus 1 (Lindsayitform) dasjenige mit den $Z$ willingsflächen in der Zone der Hauptaxe [(110) und (010)], für Typus 2 das Bavenoer und Manebacher Gesetz, so kommt man zu Zwillingscomplexen, welche nicht nur in Hinsicht ihrer allgemeinen Form, sondern auch den Winkeln nach sich den Grundformender allgemeiner vorkommenden Silicate nähern, mehr oder weniger, je nach der grösseren oder kleineren chemischen Aehnlichkeit zwischen diesen Mineralien und dem Anorthit. Diese Vergleichungen, welche ich durch Modelle anschaulich zu machen versucht habe, fübrten mich zu der Ansicht, dass die Grundformen oder richtiger die Grundmolekulle dieser Silicate so zusammengesetzt sind, wie die genannten, ihnen entsprechenden Zwillings- und Drillingscomplexe des Plagioklases, eine Ansicht, welche sich der von Mallard aufgestellten Theorie der anomalischen Krystalle anschliesst. Auch den von Beckenkamp (Zur Symmetrie der Krystalle, diese Zeitschr. 17 und 19) ausgesprochenen Sätzen schliesst sie sich an, indem sie zu dem Schlusse fübrt, dass die höher symmetrischen, holoëdrischen und holomorphen Krystalle aus weniger symmetrischen und hemimorphen Theilen, zwillingsartig mit einanderverbunden, bestehen.

Ich habe diese Ansicht durch Abbildungen der Grundformen einiger Silicate, in der angefubrten Weise getheilt, deutlich zu machen versucbt. So stellt Fig. 3 die halbe oder quasi-hemimorphe Grundform des Albites vor, in zwei $\mathrm{Zwillingshäften} \mathrm{getheilt} \mathrm{und} \mathrm{von} \infty P, O P$ und $+P \infty$ begrenzt; Fig. 4 die aus zwei hemimorphen Partialformen bestehende holomorphe Grundform :des !Natrolithes, jede aus vier Theilen bestehend und einem 
Bavenoer Vierlingscomplexe der Anorthoitform entsprechend, jedoch mit der Fläche (100) an Stelle der Fläche (110); Fig. 5 eine hemimorphe tetragonale Grundform (von Idokras), einem Achtlingscomplex der Anorthoitform (Fig. 2) entsprechend; Fig. 6 zwei hemimorphe rhomboudrische Formencomplexe, jeder einem Drillingscomplexe der Lindsayilform (Fig. 1), mit den Brachypinakoid- und Prismenflächen als Zwillingsebenen, entsprechend.

Wenn nun die einzelnen Partialformen oder Partialmoleküle zu einem homogenen Ganzen vereinigt sind, wie man es für einige anomale Silicate bei höherer Temperatur anzunehmen hat, so befindet sich der Krystall in normalem Zustande; tritt aber, gewöhnlich bei Abnahme der Temperatur, eine Sonderung der einzelnen Theile ein, durch eine im Allgemeinen geringe Veränderung in der gegenseitigen Lage ihrer Flächen, so zeigt der Krystall die anomalen krystallographischen und optischen Erscheinungen. Dieses kann nur durch eine nähere Betrachtung der inneren Molekularconstitution deutlich werden. Doch ist es zuerst nöthig, die Krystallformen im Grossen und Ganzen zu betrachten, und dabei von den Feldspathformen auszugehen.

Die Feldspathformen haben drei Hauptzonen: die prismatische oder $c$-Zone, die klino- resp. brachydiagonale $a$-Zone und die ortho- resp. makrodiagonale $b$-Zone. Diese Zonen können auch als resp. hexagonale, tetragonale und rhombische Zonen bezeichnet werden, weil die nach diesen Zonen verlängerten Krystalle, besonders ihre Zwillingscomplexe, hexagonalen, tetragonalen oder rhombischen Formen ähnlich sind. In Uebereinstimmung hiermit kann man die Silicate in drei Abtheilungen theilen je nach ihrer Aehnlichkeit in einer Hauptzone mit einer der drei Zonen des Feldspathes.

Von den Silicaten der ersten Zone oder denjenigen, deren Prismenzone in Winkeln und Ausbildung (Flächenfolge) Aehnlichkeit mit der des Feldspathes zeigt, wollen wir hier die Grundformen des Cordierits, Turmalins, Nephelins und der Glimmerarten etwas näher betrachten. Zu der Grundform des Cordierits kommt man von der des Albits (Fig. 3) und der damit analogen des Orthoklases, wenn man den vorderen Theil dieser mit einem gleichen zwillingsartig vereinigt denkt. Die gegen einander stehenden basischen Flächen der zwei Partialformen des Orthoklases bilden nämlich mit einander einen Winkel $\left(52^{0} 6^{\prime}\right)$, welcher sich dem Winkel $(102):(10 \overline{2})=$ $50^{\circ}$ 52' des Cordierits nähert, d. h. dem Polkantenwinkel des Makrodomas $\frac{1}{2} P \infty$, welches als eine Hauptform für die dem genannten Formencomplexe des Orthoklases entsprechende hemimorphe Grundform des Cordierits betrachtet werden kann.

Die Grundformen des Turmalins und Nephelins können mit einem hemimorphen Drillingscomplexe des Anorthits resp. Albits verglichen werden, welcher dem halben Formencomplexe Fig. 6 entspricht. Je nachdem 
man die hemimorphen Drillingscomplexe des Plagioklases aus drei einfachen triklinen Individuen oder drei Albitzwillingen, also aus sechs einfachen, hemiëdrisch-triklinen Partialformen bestehend denkt, kommt man $\mathrm{zu}$ einem pseudotetartoëdrischen oder einem pseudohemiëdrischen hexagonalen Formencomplex mit verschiedenen Winkeln zwischen den basischen Flächen der mit den Prismenflächen einander berührenden Partialformen $\left(44^{0} 47^{\prime}, 48^{0} 20^{\prime}, 410^{0} 20^{\prime}\right)$, welche Winkel sich den entsprechenden Grundrhomboèderwinkeln des Turmalins ( $=46^{\circ} 57^{\prime}-45^{\circ} 53^{\prime}$ nach Brooke) und des Nephelins $\left(=40^{\circ} 43^{\prime}\right)$ nähern. Man erhält hierdurch auch eine Erklärung uber die am Turmalin und Nephelin nachgewiesene Tetartoëdrie resp. Hemièdrie nebst Hemimorphie, und ihre asymmetrischen Aetzfiguren, und weiter uber die trigonalen Prismen des hemimorphen Turmalins. Wenn man nämlich den in Fig. 6 dargestellten hexagonalen Formencomplex oder den entsprechenden Zwillingscomplex des Anorthits in seine zwei hemimorphen Partialformen theilt, so werden die Prismenkanten, welche an der holomorphen Form gleich sind, verschieden, je nachdem sie den makropinakoidalen oder den brachyprismatischen Flächen des Plagioklascomplexes entsprechen; und die diesen Kanten und Flächen entsprechenden Protound Diprismen des hemimorphen Turmalins müssen also ebenfalls in zwei Hälften zerfallen. Auch die von mehreren Forschern (Breithaupt, Des Gloizeaux, Jensch, Mallard u. a.) gefundene Theilung des Turmalinrhomboëders in drei optisch zweiaxige Theile erhält hierdurch ihre Erklärung.

Im Allgemeinen kann man, wie ich in der Einleitung meiner oben citirten Abhandlung gezeigt habe, auf eine analoge Weise die von Gadolin u. a. aufgestellten Abtheilungen der Krystallformen erklären, indem man sich die Grundformen resp. Grundmolekulle als zusammengesetzt aus weniger symmetrischen Partialformen in molekularer Zwillingsverwachsung vorstellt. So kommt man von den hemiëdrisch-triklinen Formen zu dem monoklinen Systeme, von diesem zu dem rhombischen, und weiter entweder durch bexagonale oder tetragonale Formencomplexe schliesslich zu den regulären Formen. Man kann hierbei zwei verschiedene molekulare Structurformen annehmen : entweder sind die so zusammengesetzten Moleküle in dem normalen Zustande einander gleich, sondern sich jedoch in dem anomalen und bilden Zwillingslamellen, wie z. B. Leucit, oder die Partialmoleküle schliessen sich einem oder einigen zusammengeselzten Centralmolekulen symmetrisch nach allen Seiten an, so dass der Krystall in anomalem Zustande im Ganzen eine Theilung in einzelne Theile zeigt, ähnlich denen des Centralmoleküls, so z. B. anomaler Granat, Idokras u. a., welche auf den Hauptflächen die sogenannte Stundenglas-Structur zeigen. Wie besonders Klein gezeigt hat, steht diese Structur in naher Beziehung zu den äusseren Krystallfächen, obne jedoch auf diesen zu beruhen, da 
im Gegentheile angenommen werden muss, dass diese letzteren von der Molekularstructur abhängig sind.

Noch kann hier bemerkt werden, dass die auf oben genannte Weise erhaltenen Zwillingsformen des Plagioklases hemimorph sind, aber holomorph werden, wenn zwei hemimorphe Formen sich aneinander gesetzt haben. In Uebereinstimmung hiermit sind die Grundformen der krystallisirten Mineralien im Allgemeinen zweierlei : die der aufgewachsenen und die der eingewachsenen Krystalle, welche, wie ihre vorherrschenden Flächen zeigen, sich zu einander wie die genannten hemimorphen zu den holomorphen Formen verhalten, indem ibre Hauptaxen sich wie 1:2 verhalten. So zeigen z. B. die in der Richtung der Hauptaxe aufgewachsenen Krystalle des Orthoklases gewöhnlich das Doma $\mathbb{P}_{\infty}(x)$, die eingewachsenen $2 \mathbf{2 P}_{\infty}$ $(y)$; die Krystalle des aufgewachsenen Titanits die nach Dana's Auffassung positive Pyramide $P(l)$, die des eingewachsenen die negative Pyramide -2P $(n)$; die von G. vom Rath (diese Zeitschr. 5, 258) beobachteten aufwachsenen Krystalle des Dioptases von Copiapo in Chile das Rhomboèder $R$, die eingewachsenen, vollständig ausgebildeten Dioptaskrystalle von der Kirgisensteppe das Rhomboëder - $2 R$.

Wenn man die oben angeführten, mit den Cordierit- und Turmalinformen analogen, pseudorhombischen resp. pseudorhomboëdrischen $Z$ willingscomplexe des Feldspathes dreimal in der Richtung der Hauptaxe verlängert, indem man sich drei solche Complexe ubereinander gesetzt vorstellt, so kommt man zu den Grundformen des klinorhombischen Muscovits und des klinorhomboëdrischen Biotits, deren Hauptaxen, wenn man sie als monoklinisch betrachtet, dreimal so gross als diejenige des Orthoklases sind, was zu der Annahme fuhrt, dass die Grundmolekule der Glimmerarten aus drei in der Hauptaxe vereinigten einzelnen Molekulen bestehen, wobei zu bemerken ist, dass man auch hier zu zwei Grundformen, der hemi- und der holomorphen Form, kommt, je nachdem man die Pyramide $o$ oder $M$ als Grundpyramide betrachtet. Im vorigen Falle erhält man als Hauptaxe $c=1,65(=3,055)$, im letzteren $c=3,3(=3 \cdot 1,1)$ oder dreimal die $c$-Axen der Grundformen der auf-, resp. der eingewachsenen Orthoklase.

Diese bedeutende Grösse der Hauptaxe des Glimmers erklärt seine höchst vollkommene basische Spaltbarkeit, indem man a priori voraussetzen kann, dass Moleküle, welche 3-4, oder bei rhomboëdrischer Ausbildung 4-5mal so gross in der Richtung der Hauptaxe, wie in der dagegen normalen Richtung sind, mil viel grösserer Stärke in dieser als in jener Richtung zusammengehalten werden müssen. Die bedeutende Stärke der Molekularattraction des Glimmers in der Richtung der basischen Fläche zeigt sich auch durch den Einfluss, welchen sie austibt auf die darin eingeschlossenen Mineralien, wie z. B. die in Muscovit eingewachsenen Turmalin- 
krystalle, deren Prismenfläche parallel mit der basischen Fläche des Glimmers ausgedehnt ist.

. Etwas weniger lang sind die Hauptaxen der Ghloritarten: $c=\mathbf{3}, 5$ für den rhombischen Pennin und 2,277 für den klinohexagonalen Klinochlor, oder ungefähr zweimal so gross wie die des Orthoklases, welches zur Annahme eines Doppelmolekuls für den Chlorit führt. Dieses steht mit seiner weniger deutlichen Spaltbarkeit und seiner schwächeren Doppelbrechung im Vergleiche mit dem Glimmer in Uebereinstimmung.

Dass man in der That für die ungleichaxigen, nicht regulären Krystalle als ein allgemeines empirisches Gesetz den Satz aufstellen kann, dass die Molekularattraction in umgekehrtem Verhältnisse zu den Axenlängen oder im Allgemeinen den Flächennormalen steht, welche letztere den relativen Abständen der Moleküle entsprechen, das zeigŁ eine Zusammenstellung der tetragonalen und hexagonalen Mineralien nach ihren Hauptaxen. So zeigen Skapolith und Idokras mit $c=0,4-0,5$ prismatische Ausbildung und Spaltbarkeit; Zirkon, Rutil,-Cassiterit mit $c=0,6-0,7$ theils prismatischen, theils pyramidalen Habitus; Mellit, Braunit, Hausmannit mit $c=0,8-1,1$ pyramidale Ausbildung; Apophyllit, Wulfenit, Anatas mit $c=1,2-1,8$ theils einen pyramidalen, theils tafelförmigen Typus; Ghalkolit mit $c=2,06$ dunn tafelarlige Krystalle mit höchst vollkommener basischer Spaltbarkeit. So zeigen weiter E p id o tkrystalle, deren Symmetrieaxe ( $b$-Axe) kleiner als die anderen ist, Ausbildung und Spaltbarkeit parallel dieser Axe, während Krystalle von Gyps und Heulandit, deren $b$-Axe ungefähr doppelt so gross als die anderen Axen, nach der Symmetrieebene sebr vollkommen spaltbar und oft lafelförmig ausgedehnt sind.

Dieses Gesetz ist, obgleich anders ausgedrückt, ubereinstimmend mit dem von Frankenheim und Bravais auf theoretischem Wege gefundenen Verhältnisse zwischen Spaltbarkeit und Molekularstructur. Die scheinbaren Ausnahmen dieser allgemeinen Regel beruhen wohl auf dem Unterschiede zwischen physikalischen Molekülen (Grundmolekülen), welche die Spaltbarkeit bedingen und welche aus den chemischen Molekülen zusammengesetzt sind, und den aus diesen physikalischen Molekulen zusammengesetzten Krystallmolekulen, welche die Ausbildung bedingen und auf welchen zum Theil die äusseren Medien Einfluss haben.

Die Glimmerarten zeigen deutlich, dass in der Natur keine scharfen Grenzen zwischen den Krystallsystemen existiren. Biotit (= Meroxen und Phlogopil) kann man zum Theil als rhomboëdrisch mit monokliner Anomalie, oder wie Naumann es nannte, monokliner Meroëdrie betrachten, zum Theil als wirklich monoklin vom Anfange an, aber mit hexagonaler Formenausbildung. Dass der eigentliche Biotit (Meroxen) wenigstens zum Theil bei seiner Bildung, d. b. bei höherer Temperatur normal rhomboedrisch 
gewesen ist, scheint mir aus seinem geringen optischen Axenwinkel und dessen Verminderung (nach Des Cloizeaux) hei Temperaturerhöhung hervorzugehen und weiter aus den rhomboëdrischen Aetzfiguren des Phlogopits. Diese Glimmerart zeigt, wie ich an mit Fluorwasserstoffsäure geälztem Phlogopit von Pargas gefunden habe, trigonale Aetzfiguren, oft von hexagonalen Umrissen umgeben, welche zu den trigonalen Seiten in derselben Stellung stehen wie die Prismenflächen zu den pseudorhomboidischen Flächen der oben genannten Drillingsformen des Plagioklases (siehe Fig. 6). Auf eine Analogie zwischen den Plagioklasdrillingen und den Grundformen des $M g$-Glimmers verweisen auch die Schlag- und Drucklinien des letzteren, von welchen jene mit den Deuteroprismenflächen, diese mit den Pseudorhomboëderflächen der Molekularformen parallel gehen.

Die Sprödglimmer-Arten (Clintonit von New York und Waluewit vom Ural) geben mit Fluorwasserstoffsäure, wie ich gefunden habe, ebenfalls trigonale Aetzfiguren, deren Seiten aber zu den Schlaglinien nicbt normal, wie bei Phlogopit, sondern parallel gehen, und welche nicht so deutlich monosymmetrisch ausgebildet sind, wie diejenigen des Phlogopits. Zum Theil sind auch diese trigonalen Figuren von hexagonalen Aetzfiguren umgeben, den Schlaglinien jedoch nicht parallel, sondern zu ihnen normal orientirt und also nicht Deutero-, sondern Protoprismenflächen entsprechend. Dieses deutet rhomboëdrische oder quasi-rbomboëdrische Molekularformen an, in verwendeter Stellung zu derjenigen des Phlogopits, mit den Prismenseiten den Flächen des hexagonalen Protoprisma entsprechend. Denkt man sich die zwei hemimorphen Theile der Fig. 6 oder des damit analogen Drillingscomplexes des Anorthits so aneinander gestellt, dass die gleichen Kanten einander entsprechen, welches man durch eine Drehung des einen Theiles um $60^{\circ}$ im Verhältniss zu den anderen zu Stande bringt, und stellt man dabei den Complex so, dass seine Prismenflächen nicht den Flächen des Deutero-, sondern des Protoprismas entsprechen, so kommt er in eine Stellung, welche sich zu der vorigen (Fig. 6) verhält, wie die Aetzfiguren des Sprödglimmers zu denen des gewöhnlichen Glimmers, aber sie entsprechen nun nicht mehr einem rhomboëdrisch-, sondern trapezoëdrisch-tetartoëdrischen Formencomplexe. In Uebereinstimmung hiermit scheinen mir die trigonalen Aetzfiguren und die entsprechenden Flächen des Sprödglimmers nichl als Rhomboëder, sondern als trigonale Pyramiden resp. als Trapezoëder gedeutet werden zu müssen.

Gehen wir nun zu den Silicaten über, deren Grundformen sich von Plagioklaszwillingen mit Zwillingsebenen in der brachydiagonalen (quasitetragonalen) Zone herleiten lassen, so haben wir hier diejenigen der Pyroxen-Amphibol- und die der Andalusit-Staurolith-Gruppen zu betrachten, welche Silicate mit einander analog sind, indem das Amphibolprisma sich zum Pyroxenprisma wie das des Stauroliths zu dem des Anda- 
lusits verhält. Der Bavenoer $Z$ willing der Anorthitform (Fig. 2), nach der Domenfläche (0त्21) als $Z$ willingsebene, ist ähnlich einer monosymmetrischen Form mit den Winkeln $(040):(010)^{\prime}=93^{0} 36^{\prime}$ und $(040):(140)=58^{\circ} 4^{\prime}$, welche sich den entsprechenden Winkeln des Pyroxens (110): $(1 \bar{T} 0)=92^{\circ}{ }^{\circ} 4^{\prime}$ und (T10):(T11) $=58^{0} 49^{\prime}$ nähern. Die Grundform des Andalusits wie auch die des rhombischen Pyroxens müssen dagegen mit einem Doppelzwilling oder Bavenoer Vierling des Anorthits (ähnlich Fig. 3) verglicben werden.

Man kann in Uebereinstimmung hiermit die halben resp. hemimorphen Grundformen des Pyroxens und Andalusits als zwei resp. vier hemiëdrischtriklinen Partialformen entsprechend betrachten, und erhält dadurch eine Erklärung der triklinen Anomalie, welche die monoklinen Pyroxenkrystalle zuweilen zeigen, so z. B., wie ich gefunden habe, die schwarzen Augitkrystalle von Pargas, welche gewöhnlich eine schiefe trikline Ausbildung und einen kleinen Unterschied der Winkel zwischen den Flächen des Prismas auf jeder Seite der Symmetrieebene zeigen. Auch kann man dadurch eine Erklärung der zwillingsartigen Viertheilung des Chiastoliths erhalten. Aehnlich der Grundform des Andalusits ist auch die des rhombischen Pyroxens im aufgewachsenen, hemimorphen Zustande als aus vier triklinen oder zwei monoklinen Zwillingstheilen bestehend anzusehen, und man kommt so zu einer Annahme über das Verhältniss des rhombischen und monoklinen Pyroxens abnlich der von Groth (Tabel]. Uebersicht der Mineral. S. 135) ausgesprochenen Ansicht.

Die den genannten zwei Gruppen angehörenden asymmetrischen Silicate Rhodonit und Cyanit sind in Hinsicht ihrer Grundformen nicht mit Anorthitzwillingen, sondern mit der einzelnen Anorthitform zu vergleichen, und können wie diese als hemiëdrisch-triklin, und also gleich der Hälfte der monoklinen oder dem Viertel der rbombischen Pyroxenform betrachlet werden.

Die naturlichste Auffassung der Grundform des Rhodonits scheint mir die von Hamberg vorgeschlagene zu sein (s. diese Zeitschr. 23, 160 f.); nach dieser ist der Rhodonit mit dem Wollastonit zu vergleichen, und die vorherrschenden Flächen $(c, b, k, n)$ nicht als Prismen- und Pyramiden-, sondern als Pinakoid- und Domenflächen zu betrachten. Aher die Grundform des Wollastonits ist meiner Meinung nach als die Hälfte von der des Alkalipyroxens (Spodumen) in der Symmetrieaxe, d. h. als hemimorphmonoklin zu betrachten, was durch das Axenverhältniss $a: b: c=$ $1,114: 0,5: 0,483 ; \beta=69048^{\prime}$ ausgedrückt werden kann; daraus erklärt sich die tafelartige Ausbildung und die Spaltbarkeit parallel mit dem Orthopinakoid, in Uebereinstimmung mit oben angefuhrtem empirischen Gesetze. Dieses Axenverhältniss ist $=a: \frac{1}{2} b: \frac{3}{4} c$ im Verhältniss zu dem des Spodumens, wenn man dieses $=1,124: 1: 0,64\left(\beta=69^{\circ} 40^{\prime}\right)$ setzt. Diese beiden nahe verwandten Silicate unterscheiden sich auch durch ihre pina- 
koidale Ausbildung und der von Frankenheim sogenannten "normalen " Flächenfolge in der Prismenzone $(\{110\},\{120\},\{130\}$ ele.) von der prismatischen Ausbildung und »anormalen « Flächenanordnung des Pyroxens und Amphibols: $(\{110\},\{130\},\{150\})$ etc.; wenn man fur diese letzteren schiefe, den Prismenflächen $(\infty P)$ parallel gehende Axen annimmt, so erhalten auch sie eine "normale " Flächenfolge. Dieses deutet einen Unterschied in den molekularen Attractionsrichtungen an, dass diese nämlich bei Spodumen und Wollastonit den Pinakoiden, bei Augit und Hornblende den Prismenflächen parallel gehen.

Die hemimorphe Grundform des Wollastonits nähert sich der hemiëdrischen Anorthoitform (Fig. 2), und kann wie diese mimetisch-tetragonale Zwillingscomplexe, ähnlich Fig. 5, zusammensetzen. Der Spodumen kann dagegen mit dem Orthoklas auf folgende Weise verglichen werden:

Spodumen :

$$
\begin{aligned}
& (011):(0 \pi 1)=61050^{\prime} \\
& (010):(100)=90 \quad 0 \\
& (001):(100)=6940
\end{aligned}
$$

Orthoklas :

$$
\begin{aligned}
& (110):(1 T 0)=61012 \\
& (010):(001)=900 \\
& (100):(001)=6357
\end{aligned}
$$

Der Spodumen zeigt also mit dem Orthoklas eine vollständige Analogie in zwei Hauptzonen, eine unvollständige in der dritten, was unten (Abth. III) seine Erklärung erhält.

An den Spodumen schliesst sich der Petalit, dessen Axenverhältniss $a: b: c=1,1.5: 1: 1,49\left(\beta=673^{\circ}\right)$ ist; er hat also eine ungefähr doppelt so grosse Hauptaxe wie die des Spodumens, welches seine verschiedene Spaltbarkeit erklärt. Der Spodumen und der Petalit stehen in Hinsicht der $c$-Axe zu einander in demselben Verhällnisse wie Pyroxen und Amphibol in Hinsicht der $b$-Axe, was mit dem Verhältnisse ihres Molekularvolumens ubereinstimmt, indem dieses für Pyroxen (Diopsid) und Amphibol (Tremolit) $=2: 4,3$, fur Spodumen und Petalit $=1: 2,1$ ist $(\mathbf{R}$ amm e l sberg, Handb. der Mineralchemie 1, 98 u. 99).

Dem Spodumen und dem Wollastonit schliessen sich weiter folgende, in krystallochemischer Hinsicht nahe verwandten Silicate an:

$$
\begin{array}{lcl}
\text { Danburit } & a: b: c=1: 0,9183: 0,441 *), \\
\text { Leukophan } & - & =1: 0,9939: 3 \cdot 0,448(=2 \cdot 0,672), \\
\text { Melinophan } & - & =1: 1: 3 \cdot 0,435(=2 \cdot 0,658), \\
\text { Skapolith } & - & =1: 1: 0,4398 .
\end{array}
$$

Ich habe hier fưr Leukophan an Stelle der von Brögger (diese Zeitschr. 16, 293) als Grundform gewählten Pyramide $p$ die öfter vorkommende Pyra-

*) Die längere Nebenaxe ist bier als $a$-Axe, der Klinodiagonale $a$ des Pyroxens entsprechend gesetzt, um die Analogie mit den oben angeführten pyroxenartigen Silicaten anzuzeigen. 
mide $x$ ( $=2 P$ nach B rög g er) als Grundpyramide betrachtet, und übereinstimmend hiermit für Melinophan die von Brögger als Deuteropyramide $2 P \infty$ angesehene Form $=P \infty$ gesetzt. Die Axenverhältnisse dieser Mineralien stehen dann in Uebereinstimmung mit dem oben angeführten empirischen Gesetze, indem die säulenförmigen und prismatisch spaltbaren Mineralien Danburit und Skapolith eine dreimal kleinere $c$-Axe besitzen, als die basisch spaltbaren und gewöhnlich tafelförmigen Leukophan und Melinophan.

Brögger betrachtel Melinophan als »tetragonal-rbombotyp-tetartoëdrisch «. Diese Abtheilung ist jedoch eine von denen, welche Gadolin (l. c.) aus der Reibe der Krystallsysteme ausschliesst, und mit der sphenoidisch-rhombischen Abtheilung vereinigt. Melinophan gehört dann demselben Systeme an wie Leukophan, aber mit noch mehr tetragonalähnlicher Form. Er verhält sich also zu Leukophan wie Thomsenolith zu Pachnolith, und seine Form zum tetragonalen Systeme wie die des monoklinen Biotit und Klinochlor zu dem hexagonalen, von der letzlere auch von Schraul als klinohexagonal bezeichnet worden ist.

Die von Schuster am Danburit nachgewiesenen Vicinalflächen und die von Brögger und Groth beobachteten Zwillingscomplexe des Leukophans und Melinophans nebst ihren asymmetrischen Aetzfiguren und optischen Verhältnissen zeigen eine innere Structur an ähnlich den Bavenoer und Manebacher Zwillingscomplexen des Plagioklases. Noch mehr als diese Silicate nähern sich die Grundform des Skapoliths und die an diese sich anschliessende Grundform des Vesuvians (Fig. 5) einem aus vier Bavenoer Zwillingen des Plagioklases bestehenden Formencomplexe. Je nachdem dieser Zwillingscomplex nur aus der einen (rechten) Hälfte der Grundform des Anorthits (Fig. 2) oder daneben aus der anderen (linken) enantiomorphen Gegenform bestebt, erhält man den Pyramidenwinkel (111):(1T1) des Vesuvians $\left(=50^{\circ} 2^{\prime}-50^{\circ} 40^{\prime}\right)$ und des Skapoliths $\left(=43^{\circ} 49^{\prime}\right)$ aus den entsprechenden Winkeln des Anorthitcomplexes $=48^{\circ} 44^{\prime}$ oder $44^{\circ} 47^{\prime}$ bis $41^{\circ} 30^{\prime}$, welchen sich auch die entsprechenden Winkel des Meliliths $\left(=45^{\circ}\right)$ und Gehlenits $\left(=40^{\circ} 46^{\prime}\right)$ nähern. Dabei muss bemerkt werden, dass der Winkel (111):(TT1) des Skapoliths (=63042') sich zu demjenigen des Gehlenits $\left(=59^{\circ}\right)$ verbält, wie dieser zu dem Winkel $(101):(T 01)$ des Vesuvians ( $\left.56^{0} 31^{\prime}\right)$, was zu der Annahme führt, dass die Zone $[100 ; 101]$ des letzteren der Zone $[110,111]$ des Skapoliths entspricht.

Weitere Belege für die Ansicht der Analogie zwischen der Molekularstruclur dieser Silicate mit den genannten Zwillingscomplexen des Plagioklases kann man darin finden, dass die entsprechenden Zonen [331, 111] der ersteren und $[130,110]$ des letzteren eine ähnliche ("anormale (c) Ausbildung haben; und ebenso ist die prismatische Zone $[110,1 \bar{T} 0]$ der vorigen der entsprechenden brachydiagonalen Zone $[010,001]$ des Plagioklases ähn- 
lich (nnormal () ausgebildet, indem in diesen letzteren Zonen Flachen mit dem Parameter 2 nachst den mit 1 folgen. Auch erhät man dadurch eine Erkliirung der krystallographischen und optischen Anomalien, welche besonders der Vesuvian im hohen Grade zeigt, und weiter über die Pseudomorphosen (resp. Paramorphosen) von Plagioklas nach Skapolith ("Paläoalbit “ Scheerer's) und von Skapolith nach Plagioklas, z. B. dem oben genannten Anorthit (Anorthoit) von Sillböle, welcher, wie ich gefunden habe (l. c.), zum grössten Theile in Skapolith (Paranthit) durch eine Structurmetamorphose verwandelt worden ist. Der Skapolith kann also als eine polymere Modification des Plagioklases betrachtet werden, wie schon fruher von Scheerer angenommen worden ist.

Schliesslich kann auch die pyramidale Hemiëdrie, welche die eingewachsenen Skapolitbkrystalle, z. B. die von Pargas zeigen, erklärt werden, wenn man sich vorstellt, dass die acht Partialmoleküle des holomorphen Skapolithmolekuls nicht Anorthitzwillingen, sondern einzelnen Anorthitformen (Fig. 2) entsprechen, in welchem Falle nur die abwechselnden, den dipyramidalen Flächen des Skapoliths entsprechenden Kanten zwischen den Pinakoid- und Prismenflächen des Zwillingscomplexes einander gleich sind, und eine solche Pyramide also in zwei Tritopyramiden zerfallen muss.

Zu der dritten Abtheilung der Silicate, nämlich denjenigen, welche Aehnlichkeit der Winkel einer Hauptzone mit den der ortho- resp. makrodiagonalen Zone des Feldspaths zeigen, gehören unter anderen folgende rhombische und monokline Silicate:

\begin{tabular}{|c|c|}
\hline$Z_{0}$ is it & $(110):(T 10)=63^{0} 34 !^{\prime}$ \\
\hline Epidot & $(100):(001)=64: 36$ \\
\hline Orthit & $(100):(001)=64.59$ \\
\hline Gadolinit & $(110):(\overline{1} 10)=64 \cdot 11$ \\
\hline Datolith & $(110):(T 10)=6438$ \\
\hline Homilit & $(110):(\bar{T} 10)=64 \quad 0$ \\
\hline Euklas & $(110):(T 10)=65$ \\
\hline
\end{tabular}

Diese Winkel nähern sich den Winkeln (100):(001) des Feldspaths (=640 4' für Anorthit, $63^{\circ} 57^{\prime}$ für Or(hoklas), wobei noch bemerkt werden kann; dass $\frac{1}{3} a: \frac{1}{3} c=0,527: 0,602$ für Epidot sich dem Verhälınisse $c: a=0,55: 0,63$ für Anortbit nähert, und dass also das Orthopinakoid $(T)$ des Epidols der basischen Fläche $(P)$ des Feldspaths entspricht.

Dass diese Silicate auch in den übrigen Zonen einander ähnlich sind, zeigt folgende Zusammenstellung ihrer Axenverhältnisse:

$\begin{array}{lclll}\text { Zoisit } & a: b: c=0,6196: 1: 0,6859 ; & \beta=90^{\circ} 0^{\prime} \\ \text { Gadolinit } & - & =0,627: 1: 0,643 ; & \beta=8927 \\ \text { Datolith } & - & =0,633: 1: 0,635 ; & \beta=8951 \\ \text { Homilit } & - & =0,624: 1: 0,641 ; & \beta=8921 \\ \text { Euklas } & - & =0,63: 1: 0,632 ; & \beta=8838\end{array}$


Das Axenverhältniss des Euklases ist das von $\mathrm{R}$ ammelsber $\mathrm{g}$ vorgeschlagene, welches jedoch weniger natürlich als das von $S c h a b u s$ zu sein scheint. Aber auch bei der Annahme dieses letzteren kann man eine Beziehung zum Epidot finden, wenn man die Flächen $(100):(001)=79^{\circ} 44^{\prime}$ bei Euklas mit den Flächen $(101):(T 01)=81^{0} 36^{\prime}$ bei Epidot parallelisirt. Dass diese Flächenrichtungen oder die damit parallel gehenden Diagonalen des rhomboidischen, klinopinakoidalen Durchschnittes des Epidots, dessen Seiten $a: c=1,58: 1,806$ ist, eine morphologische und wohl auch krystallochemische Bedeutung haben, scheint aus den von W. Ramsay gefundenen, damit nahe parallel gehenden Absorptionsrichtungen hervorzugehen. Auch entsprechen, nach der von D a n a (Min. כ. Edit. 292) gemachten Vergleichung der Epidot- und Zoisitformen, bei Zoisit, Gadolinit, Datolith, Homilit die $a$ - und $b$-Axen den genannten Diagonalen der Symmetrieebene der Grundform bei den isomorphen Mineralien Epidot und Orthit.

An die Zoisit-Euklasreihe schliesst sich auch der Mosandril und o h nstrupit an (Brögger, diese Zeitschr. 16, 75), wie folgendes Axenverhältniss des letzteren zeigt: $a: b: c=1,623: 1: 1,359=1: 0,616: 0,838$ $\left(\beta=86^{0} 55^{\prime}\right)$, wonach die Klinodiagonale ( $a$-Axe) des Johnstrupits der Ortho- resp. Makrodiagonale (b-Axe) der Silicate der Zoisit-Euklasreihe und also der längeren Diagonale der Epidotform entspricht. Wenn man diese Diagonale als Zwillingsaxe und damit einen rhombischen Schnitt, gegen $(\overline{1} 01)(r)$ unter $8^{0} 4^{\prime}$ neigend, zur Zwillingsebene annimmt, kommt man zu monosymmetrischen resp. rhombischen $Z$ willingsformen, welche den Datolith- und Zoisitformen ähnlich sind. In diesen Zwillingscomplexen sind $M: M^{\prime}=69^{\circ} 24^{\prime}, T: T^{\prime}=59^{\circ} 48^{\prime}$; die Mittelzahl $=64^{0} 36^{\prime}$ nähert sich dem Prismenwinkel obiger Silicate, deren Prismenflächen also als intermediäre oder resultirende Richtungen dieser Flächenrichtungen des Epidols betrachtet werden können. Die monoklinen und rhombischen Silicatformen dieser Reihe können also betrachtet werden als zusammengesetzt aus zwei resp. vier asymmetrischen Partialformen, worauf auch ihre anomalen krystalloptischen Erscheinungen hindeuten.

$\mathrm{Zu}$ dieser dritten Abtheilung können noch folgende Silicate gezählt werden :

$\begin{array}{ll}\text { Kentrolith (rhombisch) } & (110):(1 T 0)=64^{\circ} 42^{\prime} \\ \text { Lievrit } & (110):(1 T 0)=6722 \\ \text { Titanit (monoklin) } & (110):(1 T 0)(r)=6629\end{array}$

Die krystallographische Aehnlichkeit des Tilanits (nach Rose's Grundform) und Liëvrits zeigt weiter folgende Zusammenstellung ihrer Axenverhältnisse :

Titanit $a: b: c=0,427: 1: 0,657 ; \quad \beta=85^{\circ} 22^{\prime}$

Yttrotitanit $\quad-\quad=0,430: 1: 0,649 ; \beta=8750$

Lièvit $\quad c: b: a=0,442: 1: 0,666 ; \beta=90 \quad 0$ 
Noch mehr als die bisher angeführten Silicate nähern sich den Feldspatharten in krystallographischer wie auch in chemischer Hinsicht die Z eolitbe. Wenn man zu diesen den mit Datolith verwandten Prehnit zäblt, so ist dieser ein Glied der dritten der oben genannten Abtheilungen mit dem Prismenwinkel $(110):(1 \bar{T} 0)=80^{\circ} 12^{\prime}\left(=(001):(\overline{2} 01)=80^{\circ} 22^{\prime}\right.$ bei Orthoklas), welcher Winkel sich dem Winkel $=7^{\circ} 38^{\prime}$ des Prismas $\infty \breve{P} 2$ des Datoliths nähert, und er steht dann zu Epidot in demselben Verhältnisse, wie Mosandrit resp. Johnstrupit zu den übrigen Silicaten der Reihe oder in verwendeter Stellung, mit der kürzeren Axe $a$ der längeren Axe $b$ dieser entsprechend. $\mathrm{Zu}$ der ersten Abtheilung, den Silicaten mit hexagonalem Formentypus, gehört dagegen der $\mathrm{C}$ habasit und die ubrigen damit verwandten hexagonalen Zeolithe, deren Grundformen nach den Untersuchungen von Becke, K le in und v. Las a u lx aus wenigstens sechs triklinen Individuen zusammengesetzt sind, ähnlich den oben angeführten Drillingscomplexen von Plagioklas mit (010) und (110) als Zwillingsflächen,

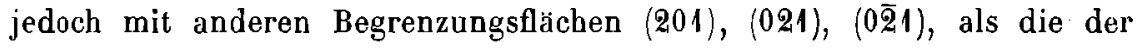
wasserfreien Silicate Nephelin, Turmalin etc. Die Mehrzahl der Zeolithe können aber zur zweiten Abtheilung gezogen werden, d. h. zu den Silicaten, deren Grundformen den Bavenoer und Manebacher Zwillingen der Feldspalharten sowohl in äusserer Ausbildung, als in innerer Structur analog sind.

Besonders auffallend zeigen die Zeolithe der Heulandit-Desmingruppe eine solche Analogie. Sie sind sämmtlich aus triklinen oder monoklinen Partialformen zu Zwillingscomplexen höherer (monoklinen, rhombischen, ja bisweilen pseudotetragonalen und regulären) Symmetrie (H armotom und Phillipsit) zusammengesetzt.

Weniger auffallend ist eine solche Zusammensetzung bei den Nadelzeolithen (Natrolith-Skolezitgruppe). Dass sie jedoch auch hier vorkommt, zeigen die Untersuchungen von Lüdecke, B rögger, Flink u. a. Wenn man sich einen hemimorphen pseudorhombischen Bavenoer Zwillingscomplex von vier Anorthit-Individuen, ähnlich der einen Hälfte der Fig. 4, vorstellt, so nähert sich dieser Vierlingscomplex den Natrolith- und Skolezitkrystallen sowohl in Hinsicht der Prismen- wie der Pyramidenwinkel. Diese letzleren sind für Skolezit $=35^{0} 40^{\prime}$ und $35^{0} 20^{\prime}$, für Natrolith $=36^{\circ} 40^{\prime}$ und $370^{\circ} 0^{\prime}$, welche Winkel sich den Winkeln (in Mittelzahl ungefähr 360) zwischen den $\{100\}$-Flachen der Partialformen des Anorthitzwillingscomplexes nähern. Der Unterschied zwischen dem rhombischen Natrolith und dem monoklinen Skolezit ist, wie im Allgemeinen zwischen nabestehenden rhombischen und monoklinen Krystallen, darin zu suchen, dass die Grundform des Natroliths aus einer doppelt so grossen Anzahl Partialformen wie die des Skolezils besteht.

Dass diese Grundformen auch hemimorph sind, zeigen sowohl in 
krystallographischer wie in physikalischer Hinsicht die Skolezilkrystalle, und bisweilen auch die Natrolithkrystalle, da sie, wie Koen en und Stad I ä n der an eingewachsenen Krystallen von Marburg gezeigt haben, aus zwei in gewendeter Stellung von $90^{\circ}$ zu einander stehenden hemimorphen Theilen bestehen, welche sich in der Mitte zu Complexen höherer teţagonaler Symmetrie zusammensetzen. In Zusammenhang hiermit kann bemerkt werden, dass man an aufgewachsenen Natrolithkrystallen aus del Auvergne eine sphenoidale Hemiëdrie constatiren kann, indem die Pyramiden regelmässig aus zwei gegenüberstehenden grösseren und zwei kleineren Flächen bestehen, und weiter, dass zwei solche Krystalle so neben einander gewachsen sind, dass die grösseren Flächen des einen den kleineren des anderen entsprechen, also in einer ähnlichen Zwillingsstellung wie die zwei Hälften der genannten eingewachsenen Krystalle. - Zu den Blätterzeolithen mit pinakoidaler Ausbildung stehen die Nadelzeolithe mit prismatischem Habitus in einem ähnlichen Verhältnisse, wie die prismatisch ausgebildeten CaMg-Pyroxene zu dem pinakoidalen Alkali-Pyroxen (Spodumen und Akmit).

Noch mehr als die vorigen Zeolithe nähert sich die Grundform des Laumontits der des Pyroxens und also auch des damit analogen Bavenoer Zwillings der Anorthoitform, deren Winkel in der Symmetrieebene (530) sich dem entsprechenden Winkel $(100):(\overline{2} 01)=54019^{\prime}$ des Laumontits nähert.

Auch die Krystalle des Ap ophyllits bestehen nach den Untersuchungen von $R u m p f$ und $K$ lein aus monoklinen resp. triklinen Theilen, welche jedoch so zusammengesetzt sind, dass sie sich als zwei rhombische Durchkreuzungszwillinge erweisen, deren Prismenflaichen den Grundpyramidenflächen des Apophyllits entsprechen, zuweilen, wie die Aetzfiguren andeuten, auch den Flächen der Deuteropyramide.

Den variirenden optischen Charakter des Apophyllits erklärt R. Braun s (Die optischen Anomalion) als beruhend auf einer Verbindung zweier in optischer Hinsicht verschiedener Varietäten; doch scheint es mir mit Rucksicht auf die einfache chemische Zusammensetzung des Apophyllits wahrscheinlicher, dass er auf einer Variation in der Molekularstructur beruht. Da nämlich die optischen Axenebenen des anomalen Apophyllits zu einander rechtwinklig liegen, so müssen die grösste und kleinste Elasticitätsaxe der Makro- resp. Brachydiagonale der rhombischen Partialformen parallel sein, und also, je nachdem die eine oder die andere von diesen in die Richtung der. Hauptaxe der Apophyllitkrystalle fällt, eine negative oder positive Doppelbrechung vorhanden sein.

An die mimetisch-regulären Krystalle des Phillipsits schliessen sich die optisch und krystallographisch anomalen regulären Zeolithe Analcim und Faujasit, welcher letztere durch seine den Oktaëderflächen vicinalen 
Ikositetraëderflichen cine aus monoklinen Zwillingscomplexen bestehende Molekularstructur anzeigt, und weiter die mehr oder weniger anomalen wasserfreien Silicate Leucit, Sodalith und Granat. Da nun zwischen den mimetischen und den mehr oder weniger krystalloptisch anomalen regulären Krystallen keine scharfe Grenze existirt, so müssen, wenn man jene als aus weniger symmetrischen Theilgestalten bestehend erkannt hat, auch diese so aufgefasst werden. Hierfür sprechen auch die krystallographischen und chemischen Beziehungen zwischen diesen regulären Krystallen und einigen von den oben betrachteten anomalen hexagonalen und tetragonalen Krystallen, so den zwischen Sodalith und Nephelin, Leucit und Skapolith, Granat zum Theil und Vesuvian. Auf Grund dieser Relationen kann der dodekaëdrische Sodalith als aus vier holomorphen rhomboëdrischen nephelinähnlichen Zwillingscomplexen (Fig. 6) zusammengesetzt betrachtet werden, wofür auch die von Brögger und Bäckström (diese Zeitschr. 18, 215) gefundenen Aetzfiguren sprechen: diese sind nämlich so angeordnet, dass jede Dodekaëderfläche von ihrer längeren Diagonale in zwei Hälften getheilt ist, was darauf hindeutet, dass der Krystall aus acht hemimorphen, vom Centrum ausgehenden rhomboëdrischen Sectoren besteht. Das Leucilmolekúl kann dagegen als zusammengesetzt zunächst aus drei holomorphen oder aus sechs bemimorphen tetragonalen Zwillingscomplexen betrachtet werden, denjenigen des Skapoliths (ähnlich Fig. 5) und also auch den entsprechenden Bavenoer Zwillingscomplexen des Anorthits ähnlich, dessen Prisnenflächen den Ikositetraëderflächen des Leucits entsprechen, und dessen Winkel (110):(110 $)^{\prime}=4^{\circ} 4^{\prime} 4^{\prime}$ den Ikositetraëderwinkeln $(112):\left(1 T_{2}\right)=48^{\circ} 12^{\prime}$ gleich sind. Dadurch erklärt sich die lamellare Zwillingsstruclur des Leucits als der des Plagioklases analog, jedoch nicht mit den Flächen des Brachypinakoides, sondern denen des Brachydomas als Zwillingsebenen, welche letzteren den Dodekaëderflächen des ganzen Complexes entsprechen. Für diese Ansicht uber das Verhältniss zwischen Leucit und Skapolith sprechen auch die von Fouqué und Michel-Lévy (Bull. soc. miner. 1880, 121; diese Zeitschr. 5, 4:15) dargestellten Leucitkrystalle, welche aus drei sich rechtwinklig durchkreuzenden, mimetisch-tetragonalen und optisch negativen einzelnen Krystallen bestehen. Auch kann hier bemerkt werden, dass die Hauptaxe des Leucitkrystalles $(c=0,52)$, als tetragonal betrachtet, sich zu der des Skapoliths $(c=0,44)$ wie die entsprechende $a$-Axe des $\mathrm{Ka} a$-Feldspaths $(=0,66)$ zu der des $\mathrm{NaCa}$-Feldspaths $(=0,63)$ verhalt.

Was schliesslich die anomalen Granatkrystalle betrifft, so können diese, wie besonders die Untersuchungen von $\mathrm{Kl}$ e in lehren, je nach ihrer verschiedenen Ausbildung betrachtet werden als bestehend aus 8 rhomboëdrischen, 12 rhombischen, 24 monoklinen oder 48 triklinen hemimorphen Partialindividuen, vom Centrum bis zu den resp. Flächen gehend. Für 
diese Ansicht spricht auch der mit Granal isomere monosymmetrische P'alchin, dessen Winkel $(110):(1 \overline{1} 0)=88^{\circ} 8^{\prime}$ und $(100):(001)=52^{0} 6^{\prime}$ den entsprechenden Winkeln des Bavenoer Anorthitzwillings $\left(8^{\circ} 6^{\circ} 4^{\prime}\right.$ und $\left.53^{0} 3^{\prime}\right)$ nahe gleich sind. Denkt man sich vier solcher Partschin-Individuen zu einem Zwillingscomplex vereinigt, der Grundform des Vesuvians mit den Winkeln $(110):(1 \overline{1} 0)=90^{\circ}$ und $(110):(111)=53^{0} 1^{\prime}$ (in Mittelzahl) ähnlich, so kommt man von dieser zu der ikositetraëdrischen Granatform auf ahnliche Weise wie oben vom Skapolith zum Leucit.

Dass man also von verschiedenen Silicaten durch wiederholte Zwillingsbildung zu mimetischen resp. anomalen regulären Formencomplexen kommen kann, deutet eine Annäherung der Formen dieser Silicate zu den regugulären Formen an, worauf besonders Mallard (Sur la quasi-identité vraisemblable de l'arrangement moléculaire dans toutes les substances cristallisées, Bull. de la soc. minéral. 7; Ref. in dieser Zeitschr. 11, 654) hingewiesen hat. Eine solche Annäherung zum regulären Krystallsysteme zeigen unter anderen die Silicate der Feldspathgruppe: wie schon Dana (A System of Mineralogy) bemerkt hat, entsprechen die Hauptflächen des Orthoklases (das Prisma, die beiden Pinakoide $0 P$ und $\infty R \infty$, und die Pyramide $+P$ ) den Flächen des regulären Rhombendodekaëders $(\infty O)$; der grösste Unterschied $\left(8^{0} 6^{\prime}\right)$ zeigt sich in der Zone der Symmetrieaxe. Aehnlichkeit damit zeigt besonders die reguläre Combination $\infty 0.0 . \infty 000.202$, wenn sie nach einer oktaëdrischen (trigonalen) Axe ausgezogen ist, wie der von Hess en berg (Mineral. Notizen Taf. IV, Fig. 20) beschriebene Sodalithkrystall vom Vesuv. Die Ursache dieser regulären Formenähnlichkeit der Krystalle im Allgemeinen muss meiner Meinung nach in der regulären oder nahe regulären Form der Elementaratome gesucht werden, worauf die meistentheils reguläre oder den regulären sich nähernde hexagonale Form der Elemente und ihrer binären Verbindungen hindeutel. Es wird also jetzt nolhwendig, sich eine Vorstellung der Constitution dieser Elementaratome zu verschaffen. Eine krystallochemische Theorie der Mineralien setzt eine solche für die chemischen Elemente voraus.

\section{II.}

Dass die sogenannten chemischen Elemente nicht solche im eigentlichen Sinne sind, sondern nur in Hinsicht ihrer noch nicht nachgewiesenen Theilbarkeit, dass sie im Gegentheile aus noch einfacheren Urelementen zusammengesetzt sind, das ist man wohl a priori berechtigt anzunehmen mil Berücksichtigung der Thatsache, dass diese Elemente in krystallographischer und physikalischer Beziehung den von ihnen zusammengesetzten chemischen Verbindungen im Allgemeinen ähnlich sind. Die sogenannten Elementaratome müssen also den von ihnen gebildeten Molekulen in der Hauptsache 
ahnlich sein, und nur durch ihre grössere Einfachheil und auf Grund davon glössere Schwierigkeit zerselzt zu werden, verschieden sein.

Schon vor mehreren Jahren habe ich einen Versuch einer systematischen Zusammenstellung der Elemente auf Grund ihrer Atomgewichte gemacht (Acta Soc. Sc. Fenn. 10), welche besser als die in chemischen Lehrbücheru dargestellten Systeme als Grundlage einer krystallochemischen Theorie und eines Systems der Mineralien dienen könte. In einem solchen Elementarsysteme müssen meiner Ansicht nach nicht nur die eigentlich chemischen, sondern auch die physikalischen Eigenschaften der Elemente bertucksichtigt werden, besonders der in mineralogischer Beziehung wichtige Unterschied zwischen dem metallischen und nichtmetallischen Gharakter. Ich vertheile deshalb die Elemente in drei Serien mit Rucksicht ihrer physikalischen Beschaffenheit: I. schwere Metalle, II. leichte Metalle nebst Metalloïden und III. die drei gasförmigen, in der Natur allgemeiner vorkommenden Elemente $H, N, O$; und es zeigt sich dann eine solche Dreitheilung auch in chemischer Hinsicht ganz naturgemäss. Ich wollte darum dieses System, im Gegensatz zu den fruberen dualistischen und der jetzigen monistischen*) Auffassung, als trialistisch bezeichnen. Folgendes Schemi ${ }^{* *}$ ) giebt eine Uebersicht dieses Elementarsystems.

\section{A. Negative Elemente.}

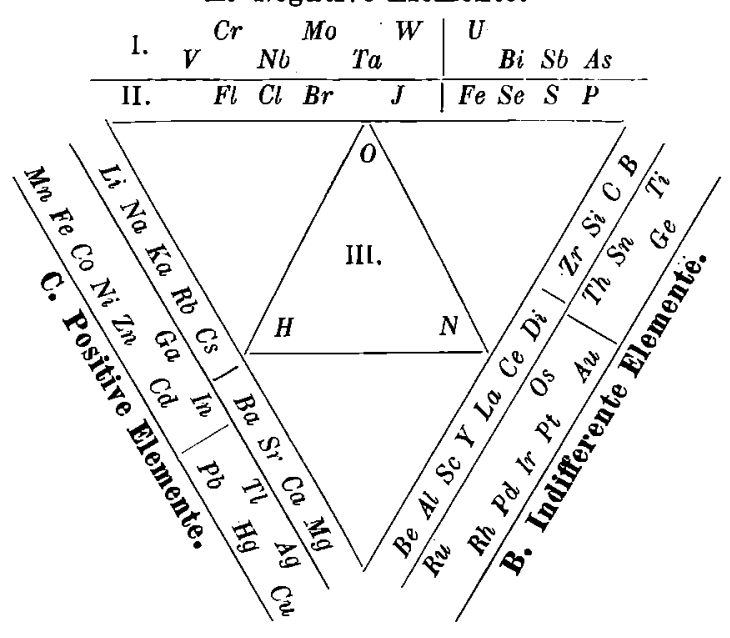

Wie man sieht, schliesst sich dieses Elementarsystem an die von Berzelius herrührende Eintheilung in elektronegative und elektropositive

*) Als ein solches scheint mir das von Mendelejeff aufgestellte System, in welchem die Elemente in einer einzigen fortlaufenden Serie einander folgen, bezeichnet werden zu können.

**) In der oben citirten Abhandlung sind die Elemente auf drei concentrischen Kreisen vertheilt. 
Elemente an, mit dem wichtigen Unterschiede, dass zwischen den negativen und positiven eine intermediäre Abtheilung vorkommt, die indifferenten oder neutralen Elemente, welche sowohl in qualitativer wie in quantitativer chemischer Hinsicht eine gleich natürliche Gruppe von Elementen wie die beiden anderen bilden, obgleich die eine Hälfte der indifferenten Serien aus schwach negativen, die andere aus schwach positiven Elementen besteht, wodurch sie einen Uebergang von den negativen zu den positiven Elementarserien bilden. Sie zeigen im Allgemeinen eine grössere chemische Trägheit oder Indifferenz als die positiven und negativen Elemente, welches sich z. B. darin zeigt, dass sie sowohl in gediegenem Zustande (die edlen Metalle) als auch in ihren Verbindungen in der Natur (die Edelsteine) eine grössere Beständigkeit als andere Elemente haben.

Dass übrigens dieses System ein natürliches genannt werden kann, zeigt sich daraus, dass die verwandten Mineralien neben einander in den einzelnen Serien zu stehen kommen, und weiter in quantitativer Beziehung darin, dass die sechs Abtheilungen (A.I und II, B.I und II, C.I und II) in Hinsicht der Atomgewichte und meistentheils auch der Atomvolumina (die Quotienten der Atom- und specifischen Gewichte) analoge Reiben bilden, indem diese Zahlen von einem Minimum an den beiden Enden der Reihe zu einem Maximum in der Mitte fortgehen, also im Ganzen eine Periodicitäl von abwechselnden Minima und Maxima zeigen, wobei zu bemerken ist, dass die schweren Metalle (Serie I) ein relativ grösseres Atomgewicht und kleineres Atomvolumen, als die entsprechenden Elemente der Serie II haben. Die Dreitheilung, welche also im Grossen und Ganzen eine natürliche genannt werden kann, zeigt sich noch weiter im Einzelnen: so z. B. kann man die Reihe A.II in drei Unterabtheilungen theilen 1) $\mathrm{Fl}, \mathrm{Cl}, \mathrm{Br}$, 2) $\mathrm{Te}$, $S e, S, 3) P$, und ebenso die Reihe G.II in drei entsprechende Abtheilungen 1) $\mathrm{Li}, \mathrm{Na}, \mathrm{Ka}, \mathrm{R} b, \mathrm{Cs}$, 2) $\mathrm{Ba}, \mathrm{Sr}, \mathrm{Ca}, 3) \mathrm{M} g$, wobei jedoch bemerkt werden kann, dass zwei von diesen Unterabtheilungen einander näher stehen, wie ja auch die grossen Abtheilungen der indifferenten und positiven Elemente einander näher stehen und zusammen einen Gegensatz zu denen der negaliven Elemente bilden.

Aber die Dreitheilung geht noch weiter, indem die Elemente, wie schon Döbere in er (Pogg. Ann. 1829, 15) bemerkt hat, sich je drei und drei zu natürlichen Triasgruppen zusammenstellen lassen. Solche »Triaden " bilden: $\mathrm{Li}, \mathrm{Na}, \mathrm{Ka} ; \mathrm{Ka}, \mathrm{R} b, \mathrm{Cs} ; \mathrm{B} a, \mathrm{Sr}, \mathrm{Ca} ; \mathrm{B} e, \mathrm{Al}, \mathrm{Sc} ; \mathrm{La}, \mathrm{Ce}, \mathrm{D} i ; \mathrm{Zr}$, $\mathrm{Si}, \mathrm{C} ; \mathrm{S}, \mathrm{Se}, \mathrm{Te} ; \mathrm{Cl}, \mathrm{Br}, \mathrm{J}$; und unter den schweren Metallen: $\mathrm{Fe}, \mathrm{Ni}$, Co; $\mathrm{P} d, \mathrm{R} h, \mathrm{Ru} ; \mathrm{P} t, \mathrm{Ir}, \mathrm{Os} ; \mathrm{Bi}, \mathrm{Sb}, \mathrm{As} ; \mathrm{Cr}, \mathrm{Mo}, \mathrm{W} ; \mathrm{V}, \mathrm{Nb}, \mathrm{Ta}$. Auch in diesen Triasgruppen stehen zwei Elemente einander näher, so z. B. Ni und $C_{0}, S b$ und $A s ; \mathrm{Nb}$ und $\mathrm{Ta}$. Eine andere Analogie in quantitativer Beziehung zeigen die Triaden der leichten Metalle und der Metalloide unter einander, indem die Atomzahl des in Hinsicht des Atomgewichtes zwischen den beiden anderen 
slehenden Elementes die Mittelzahl von diesen bildet, oder mit anderen Worlen: die Summe der Atomgewichte dieser beiden ist gleich der doppelten des Atomgewichtes des dritten, welches zuweilen erst als solches eine ganze Zahl bildet, so z. B. $R b(85,5), S r(87,5), S e(79,5)$.

Eine Ausnahme der übrigens grossen Analogie zwischen den zwei Partialserien A und B der Serie II machen die Halogene, welche nur vier, während die entsprechenden Alkalimetalle fünf sind. Man wird dadurch zu der Hypothese gefuhrt, dass ein noch nicht entdecktes, am nächsten dem Fluor sich anschliessendes negatives Element vorkommt, welches mit $F l$ (19) und $\mathrm{Cl}$ (35) eine Triade bildet, und dessen Atomgewicht also $=3$ gesetzt werden kann $(3+35=2 \cdot 19)$. Aber dieses Element weist auf zwei andere hin, deren Atomgewichte nach dem triadischen Zahlengesetze resp. 2 und 1 sind, von welchen das letztere das des positiven Wasserstoffes ist, das andere (2) also als das indifferente Glied dieser hypothetischen Elementartriade $(1,2,3)$ zu betrachten ist. Dass übrigens Elemente aus verschiedenen Serien mit einander Triaden bilden, zeigt z. B. die Triade $P(31)$, $A s(75), S b(120)$, indem $31+120=2 \cdot 75,5$ ist.

$\mathrm{Zu}$ diesen drei Elementen mit den kleinsten Atomgewichten, welche ich deshalb als die eigentlichen Urelemente bezeichnen möchte, kommt man auch, wenn man die Atomzahlen der Elemente in ihre einfachsten Theile auflöst, wie folgende Zusammenstellung einiger Elementarreihen anzeigt, welche zum Theil eine Analogie mit den organischen Kohlenwasserstoffradicalen zeigen, und wie diese als homologe Serien bezeichnet werden können.

Positive Elemente.

$$
\begin{array}{ll}
L i=6 \cdot 1+1=7 & F l=6 \cdot 3+1=19 \\
N a=6 \cdot 1+1+8 \cdot 2=23 & \left.C l=6 \cdot 3+1+8 \cdot 2=35[35,4]^{*}\right) \\
K a=6 \cdot 1+1+2(8 \cdot 2)=39 & B r=6 \cdot 3+1+4(8 \cdot 2)=83[79,8] \\
\left.R b=6 \cdot 1+1+5(8 \cdot 2)=87[85,5]^{*}\right) \quad J=6 \cdot 3+1+7(8 \cdot 2)=131[126,5] \\
C s=6 \cdot 1+1+8(8 \cdot 2)=135[133] \\
B a=6 \cdot 1+2+8(8 \cdot 2)=136[137] & T e=4 \cdot 3+2 \cdot 2+7(8 \cdot 2)=128 \\
S r=6 \cdot 1+2+5(8 \cdot 2)=88[87,5] & S e=4 \cdot 3+2 \cdot 2+4(8 \cdot 2)=80[79,5] \\
C a=6 \cdot 1+2+2(8 \cdot 2)=40 & S=4 \cdot 3+2 \cdot 2+8 \cdot 2=32 \\
M g=6 \cdot 1+2+8 \cdot 2=24 & (O=4 \cdot 3+2 \cdot 2=8 \cdot 2=16)
\end{array}
$$

*) Die zwischen Klammern gestellten Zahlen sind die empirisch gefundenen Atomgewichte, welche für einige Elemente mit grösserem Atomgewichte sich etwas von den theoretisçhen Zahlen unterscheiden, wobei jedoch bemerkt werden kann, dass diese besser als die empirischen mit dem oben angeführten triadischen Gesetze übereinstimmen; so ist $z$. B. für die Triade $C l, B r, J: 35+131=2 \cdot 83$, während $35,4+126,5=$ $2 \cdot 80,95$ ist. 


$$
\begin{array}{ll}
M n=6 \cdot 1+1+3(8 \cdot 2)=55 & V=6 \cdot 3+1+2(8 \cdot 2)=51 \\
F e=6 \cdot 1+2+3(8 \cdot 2)=56 & C r=6 \cdot 3+2+2(8 \cdot 2)=82 \\
Z n=6 \cdot 1+3+4(7 \cdot 2)=65 & A s=4 \cdot 3+3+4(7 \cdot 2)=75 \\
C u=6 \cdot 2+3+3(8 \cdot 2)=63 & P=4 \cdot 3+3+8 \cdot 2=31
\end{array}
$$

\section{Indifferente Elemente.}

$$
\begin{array}{ll}
B e=6 \cdot 1+3=9 & B=4.2+3=11 \\
A l=6.2+1+7.2=27[27,3] & C=4.3=6.2)=12 \\
(N=6.2+2=7.2=14) & S i=6.2+8 \cdot 2=28 \\
A u=6.2+2+13(7.2)=196 & T i=3.2+2+3(7.2)=50 \\
P t=6.2+13(7.2)=194 & S n=3.2+8(7.2)=118
\end{array}
$$

Die meisten von diesen Elementen zeigen nun eine solche Anzahl von den Urelementen $(1,2,3)$, dass diese sich auf einem oktaëdrischen Raume vertheilen lassen, nämlich sechs Atomzahlen von 1 für die positiven Elemente, von 2 für die indifferenten Elemente nebst einem Theil der positiven Metalle, und von 3 fur die negativen Elemente; diese sechs Uratome bilden, wenn sie auf gleicbe Abstände von einander gedacht werden, die Eckpunkte eines oktaëdrischen Raumes, in dessen Mitte das 7. Urelement (1, 2 oder 3) als Central- oder Kernatom gedacht werden kann; und auf den Oktaëderflächen können die übrigen $m \cdot 8(2)$ resp. $m \cdot 7(2)$ Atomzahlen (Multipla von den Atomgewichten des Sauerstoffes und Stickstoffes) vertheilt werden, ohne dass die oktaëdrische Form dadurch verändert wird, welches die Isomorphie der homologen Reihe erklärt; nur das Atomvolumen wird dadurch im Allgemeinen vergrössert. Hierdurch erklärt sich nun auch das von Newlands sogenannte Octavengesetz (Law of octaves) und das von Me ndel ej ef $f$ als "periodisches" Gesetz bezeichnete constante Wiederauftreten verwandter Elemente in der von ihm aufgestellten, nach den Atomgewichten fortlaufenden Elementarserie.

Auch die Atomzablen der nicht regulären Elemente, z. B. die des rhombischen Schwefels, des rhomboëdrischen Arsens, des letragonalen Zinns lassen sich, wie ich in fruheren Abhandlungen gereigt habe, in Uebereinstimmung mit den Formen dieser Elemente bringen. Da indessen diese Elemente von weniger Bedeutung für die Silicate sind, gebe ich hier nicht weiter in eine Betrachtung ihrer atomistischen Gonstitution ein.

Auch ist es unnöthig hier in eine nähere Betrachtung über die eigentliche Natur der drei Uratome einzugehen. Ich beschränke mich anzuführen, dass ich sie als Kraftcentra oder Kraftpunkte (Dynamiden) betrachte, von Aetherșphären umgeben, welche sie zusammenzufallen verbindern und welche wahrscheinlich in umgekehrtem Verhältnisse zu ihren Atomgewichten stehen, so dass die elektro-positive Dynamide (1) einen Ueberschuss, die negative (3) einen relativen Mangel an elektrisch-ätherischem Substrat hat, in Uebereinstimmung mit der von Edlund aufgestellten elektrischen 
Theorie, nach welcher die elektro-positiven und -negativen Gegensätze auf einen relaliven Ueherschuss und Mangel an elektrischem Aether beruhen. Weiter kann man sich vorstellen, dass die Dynamide 1 einen Punkt, 2 zwei Punkte oder eine Linie, 3 drei Punkte oder eine dreieckige Fläche bildet. Keine von den drei Dynamiden kann also allein einen Raum bilden, und die kleinste Anzahl raumbildender Dynamiden derselben Art ist vier, wodurch ein tetraëdrischer Raum entsteht. Einen solchen hat man für das vieratomige Kohlenstoffatom ( $4 \cdot 3$ ) anzunehmen, indem die vier Dynamiden (3) einen Raum von vier gleichseitigen Dreiecken, also ein reguläres Tetraëder bilden, eine Ansicht, welche sich der neuesten Theorie (von van't H off und Wislicenus) der organischen Chemie anschliesst.

Wie man nun durch diese Elementartheorie eine Erklärung der Atomgewichte und ihrer gegenseiligen Verhältnisse erhält, so kommt man auch dadurch zu einer einfachen Erklärung der verschiedenen chemischen Werthe der Alome. Wie man aus obiger Zusammenstellung sieht, haben die einatomigen positiven und negativen Elemente die Dynamide 1, die zweiatomigen 2, und die dreiatomigen $(B, P, A s) 3$ als Centraldynamide; und man kann deshalb annehmen, dass dieses Kernatom den ganzen oktaëdrischen oder quasi-oktaëdrischen Dynamidencomplex zwingt, mit resp. einer Ecke, einer Kante oder einer Fläche, d. h. mit 1, 2 oder 3 Dynamiden anzugreifen. Aber auch die auf den Flächen des Dynamidencomplexes vertheilten indifferenten Dynamiden (2) können wahrscheinlich einen richtenden Einfluss auf den ganzen Complex ausüben, welches die Ausnabmen und Unregelmässigkeiten der angenommenen Atomwerthe erklärt.

Die meisten Elementaratome scheinen Dynamidencomplexe mit Centraldynamiden zu sein. Zu den wenigen, für welche man kein solches Kernatom anzunehmen hat, gehören $C$ und $S i$; und bierdurch erklärt sich die grössere Freiheit und Mannigfaltigkeit, welche diese Elemenle in ihren Verbindungsverhältnissen zeigen: die grössere chemische Trägheit oder Indifferenz des Siliciums im Vergleich zum Kohlenstoff erklärt sich durch die acht indifferenten Dyamiden (2) des vorigen, welche einen oktaëdrischen Dynamidencomplex bedingen; die sechs Eckendynamiden dieses letzteren mussen so vertheilt gedacht werden, dass drei Kraftpunkte den abwechselnden (tetraëdrischen) Flächen des oktaëdrischen Complexes entsprechen, wodurch eine Analogie mit dem tetraëdrischen Kohlenstoffatom entsteht.

Schwieriger als die atomistische Constitution des Kohlenstoffes und Siliciums ist die des Aluminiums zu bestimmen, schon deswegen, weil das Atomgewicht des letzteren nicht so sicher bestimmt ist wie das der vorigen, indem die Angaben für das Alomgewicht des Aluminiums zwischen 27 und 27,5 wechseln. Wenn man diese letztere Zahl annimmt und zugleich berücksichtigt, dass Aluminium gewöhnlich als Doppelatom $\left(A l_{2}\right)$ auftritt, könnte man $A l_{2}=2(6 \cdot 2+7 \cdot 2)+3=2 \cdot 27,5$ setzen, d. h. als zwei durch 
die Dynamide 3 als Kernatom vereinigte, mit dem Siliciumatom analoge oktaëdrische D jnamidencomplexe, jedoch mit dem Unterschiede, dass nur sieben Oktaëderflächen von den intermediăren Dynamiden (2) eingenommen sind; die achte, für die zwei vereinigten Atomcomplexe gemeinsame Oktaëderfläche wird an deren Stelle von dem Kernatome (3) eingenommen. Hierdurch geht die reguläre Form der einzelnen Complexe in eine rhomboëdrische Form des Doppelcomplexes über. Solche hexagonale oder quasihexagonale Doppelatomeomplexe hat man auch für andere Elemente anzunehmen, welche wie $Z n, C u, A s, P$ die Dynamide 3 als Kerndynamide haben oder $m \cdot 7(2)$ als Flächendynamide, in welchem letzteren Falle die einfachen Atomcomplexe als hemimorph-rhomboëdrisch bezeichnet werden können. Auch die Elemente, welche wie z. B. $M n$ und $F e$ eine solche Anzahl Flächendynamiden haben, dass auf jede Oktaëderfäche 3 - 2 Dynamiden kommen, können rhomboëdrische Doppelatome $\left(R_{2}\right)$ und rhomboëdrische Oxyde $\left(\mathrm{R}_{2} \mathrm{O}_{3}\right)$ bilden.

Ein rhomboëdrischer Atomcomplex kann auch dadurch zu Stande kommen, dass drei zweiatomige Dynamidencomplexe sich mit ihren Kanten so zusammenlegen, dass drei Oktaëderflichen in eine Ebene (den basischen Flächen des ganzen Complexes entsprechend) zusammenfallen. Ein solcher Complex ist für $M g_{3}$ und $B e_{3}$ anzunehmen, wodurch sie mit $A l_{2}$ krystallochemisch äquivalent werden. In Uebereinstimmung hiermit scheint auch $O_{3}$ (Ozon) aus drei mit einander zu einem hexagonalen Tripelmolekül vereinigten einzelnen Dynamidencomplexen zu bestehen, während der einzelne Dynamidencomplex von Sauerstoff wahrscheinlich mit der basischen Mittelebene des rhombischen Schwefelatomcomplexes (siehe unten) analog ist. Mit Rücksicht hierauf kann das Wasser in seiner hexagonalen Form $=3\left(\mathrm{H}_{2} \mathrm{O}\right)$ angenommen werden.

Die allotropischen Modificationen der Elemente erklären sich also in diesen Făllen als eine Polymerie. Fur andere z. B. die des Kohlenstoffes (Diamant und Graphit) muss man wohl mit Rücksicht auf ihre ganz verschiedenen Charaktere eine wírkliche Isomerie voraussetzen, $d . h$. eine verschiedene Lagerung innerhalb des Dynamidencomplexes: so kann man das Atom oder Molekül des tetraëdrischen, nichtmetallischen Diamants als bestehend aus vier negativen Dynamiden (3), das des metallischen klinohexagonalen Graphits aus sechs indifferenten Dynamiden (2) betrachten, zu einem rhomboëdrischen Gomplex vereinigt, wobei wahrscheinlich mit Hinsicht der glimmerartigen Spaltbarkeit zwei oder drei solche chemischen Moleküle zu einem in der Hauptaxe verlängerten physikalischen Moleküle, analog mit der des Biotits, verbunden sind.

Wie nun die drei Dynamiden oder Uratome sich zu Dynamidencomplexen oder Elementaratomen zusammensetzen, so verbinden sich diese lel'zteren weiter zu zusammengesetzten Atomcomplexen oder Molekülen; und 
je nachdem die ähnlichen, meistens regulären Elementaratome in paralleler oder nichtparalleler Stellung zu einander stehen, erhält auch das so zusammengesetzle Molekül eine reguläre oder nichtreguläre, weniger symmetrische, aber gewöhnlich mehr oder weniger der regulären sich nähernde Form. In letzterem Falle können die oktaëdrischen Dynamidencomplexe drei verschiedene Hauptstellungen zu einander annehmen, welche man als tetragonale, rhombische und hexagonale unterscheiden kann, je nachdem eine quaternäre Axe (oktaëdrische Eckenaxe), binäre (oktaëdr. Kantenaxe) oder ternäre Axe (oktaëdr. Flächenaxe) aufrecht stebt oder im Allgemeinen dieselbe Richtung einnimmt. Zu diesen kommen weiter noch schiefe (monooder trikline) Stellungen. Diese verschiedenen gegenseitigen Stellungen beruhen auf der Lage der einzelnen Dynamidencomplexe zu dem Schwerpunkte oder Centrum des ganzen molekularen Kraftsystems, und jene Lage auf den verschiedenen ein-, zwei- oder dreizähligen Angriffspunkten des Complexes (Eckkanten- oder Flächenstellung).

Die einzelnen Kräfte eines solchen Kraftsystems halten einander das Gleichgewicht wie die Kräfte eines sogenannten mathematischen Hebels, d. h. die molekularen Kräfte verhalten sich umgekehrt wie ihre Abstände vom Centrum des Kraftsystems. Diese Abstände sind durch die Normalen der Richtungen der Kräfte, d. h. die Normalen der Grund- oder Hauptflächen des Krystalles reprăsentirt; und die Kräfte können also durch die reciproken Werthe dieser Normalen ausgedruckt werden. Dieser auf deductivem Wege hergeleitete Satz steht mit dem oben (Abtheil. I) angeführten empirischen Gesetze über das Verhältniss der Molekularattraction und der Flächennormalen in Uebereinstimmung.

Ich habe nun die Werthe der Normalen der Grundflächen von einer grossen Anzabl krystallisirter Mineralien aus ihren Axenelementen berechnet und dabei gefunden, dass sie in demselben Verhältnisse zu einander stehen wie das der drei Urelemente, $d$. h. wie die Zahlen 1:2:3, wie folgende Beispiele zeigen. Ueber die Bezeichnungsweise der Normalen und ihrer reciproken Werthe siehe weiter unten.

$$
\begin{aligned}
& \text { Orthoklas } a: b: c=0,6585: 1: 0,556 ; \beta=63^{0} 57^{\prime} \text {. } \\
& b^{1}(010)=1 \\
& b_{1}=1 \\
& a b^{1}(110)=\sin 30^{0} 36^{\prime}=0,509 ; \\
& a b_{1}=1,965 \\
& \bar{a} c^{1}(\bar{T} 01)=c \sin 65^{0} 46^{\prime}=0,506 \text {; } \\
& \bar{a} c_{1}=1,976 \\
& c^{1}(001)=c \sin 6357=0,499 ; \\
& c_{1}=2,004 \text {. } \\
& \text { Skapolith } a b^{1}=\sin 45^{0}=0,7071 ; \quad a b_{1}=4,414(1) \\
& a b c^{1}(111)=a b^{1} \sin 31^{\circ} 51^{\prime}=0,3647 ; a b c_{1}=2,742(1,94) \text {. } \\
& \text { Epid ot } c^{1}=c \sin 64^{\circ} 36^{\prime}=1,631 \text {; } \\
& c_{1}=0,6131(1) \\
& a b^{1}=\sin 55^{0}=0,819 \text {; } \\
& \bar{a} b c^{1}(\bar{T} 11)=\sin 54^{0} 47^{\prime}=0,817 ; \\
& a b_{1}=1,221(1,991) \\
& a b c_{1}=1,224(1,996) \text {. }
\end{aligned}
$$




$$
\begin{aligned}
& \text { Pyroxen } a b^{1}=\sin 46^{0} 27^{\prime}=0,7248 ; a b_{1}=1,377 \text { (2) } \\
& \bar{a} b c^{1}=\sin 29^{0} 36^{\prime}=0,4939 ; \quad \bar{a} b c_{1}=2,024(2,94) . \\
& \text { Barylocalcit } a: b: c=0,974: 1: 0,847 ; \beta=69^{0} 30^{\prime} \text {; } \\
& a^{1}(100)=a \sin 69^{\circ} 32^{\prime}=0,9123 ; \quad a_{1}=1,096(2) \\
& \bar{a} b c^{1}=\sin 36^{0} 32^{\prime}=0,5953 ; \quad \bar{u} b c_{1}=1,680(3,065) . \\
& \text { Tantalit } \quad a^{9} b^{4}=\sin 61^{0} 27^{\prime}=0,8784 ; \quad a_{4} b_{9}(490)=1,138(1) \\
& a b c^{1}=\sin 27^{\circ}=0,454 ; \quad a b c_{1}=2,203(1,94) . \\
& \text { Libethenit } c^{1}=1,702 \text {; } \\
& a b^{1}=\sin 43^{0} 50^{\prime}=0,6926 ; \\
& c_{1}=1,425(1) \\
& a b_{1}=1,444(1,013) \text {. } \\
& \text { Baryt } c^{1}=1,312 \% \text {; } \\
& a b^{1}=\sin 39^{0} 10^{\prime}=0,6316 ; \\
& c_{1}=0,7616 \text { (1) } \\
& a b_{1}=1,583(2,079) \text {. } \\
& \text { Markasit } c^{1}=1,1845 \text {; } \\
& a b^{1}=\sin 36^{0} 57^{\prime}=0,6011 ; \\
& c_{1}=0,8442(1) \\
& a b_{1}=1,663(1,970) \text {. } \\
& \text { Schwefel } c^{1}=1,9039 \text {; } \\
& a b^{1}=\sin 39^{0} 1^{\prime}=0,6296 ; \\
& c_{1}=0,5252(1) \\
& a b_{1}=1,588(3,024) \text {. }
\end{aligned}
$$

Wie natürlich ist, können diese Werthe nur annäherungsweise dem Zahlenverhältnisse 1:2:3 gleich sein; sie sind nicht rationale, sondern wie die Axenwerthe irrationale Zahlen, und entsprechen nicht nur den Abständen der Atome in dem Molekule, sondern auch dem der Molekule unter einander, welche letzteren nicht nur von den vorigen, sondern auch von der Temperatur bedingt sind. Aber sie entsprechen dann so viel mehr dem aus den Atomgewichten deducirten Verhältnisse der Alomkräfte; denn die Atomgewichte können gleichfalls nicht absolut genau durch rationale Zahlen ausgedrückt werden; weil nach den äusserst genauen Untersuchungen von Stas die Atomgewichte nicht rationale Zahlen sind, obgleich sie, wie $\mathrm{S}$ t a s bemerkt, so nahe einfachen ganzen Zahlen oder Zahlen mit einfachen Brüchen liegen, dass man sie bei gewöhnlichen praktischen chemischen Arbeiten als solche betrachten kann. Aber aus demselben Grunde kann man sie auch bei theoretischen Speculationen als solche annehmen, da es hier weder möglich, noch nöthig ist, eine absolut genaue, sondern nur eine approximative Uebereinstianmung $z$ wischen den theoretischen und empirischen Zahlen zu erbalten. Die Prout'sche Hypothese, dass die Atomgewichte Multipla der Atomgewichte des Wasserstoffes wären, kann jedenfalls unter solchen Bedingungen als richtig angesehen werden. Auch tbeoretisch kommt man zu der Ansicht, dass die Atomgewichte irrational sein müssen, da die Dynamiden, welche sie zusammensetzen, nicht als eínander absolut gleich angenommen werden können.

Ich betrachte aus diesem Grunde die reciproken Werthe der Normalen der Flächen der Grundform eines Krystalles als approximative Ausdrücke der Atomkräfte, als "Atommeter", um einen schon von Heinrichs und 
Schra uf für ähnliche lineare Atommasse angewendeten Namen zu benutzen (siehe S chrauf, Vergl. morphol. Studien: Ueber die axiale Lagerung der Atome in Krystallen; diese Zeitschr. 9, 265). - Dass die Atomkrafte der Elementaratome durch die Zahlenverhältnisse 1:2:3 ausgedrtickt werden können, zeigt sich aus oben angeführter Zusammenstellung der Atomgewichte der Elemente, sowohl wenn man die einzelnen, an den Ecken resp. Kanten angreifenden Dynamiden 1, 2,3, wie auch wenn man den Dynamidencomplex im Ganzen berücksichtigt, wobei jedoch nur der constante Theil desselben oder die formbildenden Dynamiden in Betracht kommen, da die Flachendynamiden (2) nicht wie diese nach aussen wirken und also als sowohl krystallographisch wie chemisch indifferent bezeichnet werden können. Zieht man also nur die formbildenden Dynamiden in Betracbt, so kommt man zum Verbältnisse $6: 12: 18=1: 2: 3$.

Zur Erläuterung dieser Vergleichung zwischen Atomkräften und den Flächennormalen der Grundform eines Krystalles mögen hier einige der oben angefübrten Mineralien dienen. Wie man aus obiger Zusammenstellung sieht, verhalten sich die reciproken Werthe der Normalen des Prismas $(a b)$ und der basischen Fläche $(c)$ bei dem rhombischen $\mathrm{Sch}$ we fel, wenn man die vorherrschende Pyramide $(P)$ als Grundpyramide betrachtet, wie die Zahlen $3: 1$, was zu der Annahme führt, dass die vier negativen Dynamiden (3) den vier Prismen-Normalen resp. -Flächen, die vier positiven Dynamiden(1) den zwei basischen Normalen resp. Flächen, und die acht indifferenten Dynamiden den acht Pyramiden flächen entsprechen. Für den Baryt kommt man zu einem ähnlichen Grundmolekul (s. diese Zeitschr. 11, 311), indem die krystallostatiscben Kräfte oder atomistischen Constanten von $S$ zu den von $B a$ sich wie 12:6=2:1=abl $: c_{1}$ verhalten, d. h. wie die reciproken Werthe der Normalen der Spaltungsflächen; und ebenso bat man für Markasit $S: F e=2: 1=a b_{1}: c_{1}$, jedoch mit dem Unterschiede, dass das physikalische Molekul des Baryts $\left(=4 \mathrm{BaSO}_{3}\right)$ aus vier, das des Markasits $\left(\mathrm{FeS}_{2}\right)$ aus zwei chemischen Molekülen besteht, was krystallochemisch so ausgedrückt werden kann, dass das Barytmolekül aus vier quasi-triklinen, das Markasitnolekül aus zwei monoklinen Partialmolekulen zusammengesetzt ist.

Eine den vorigen analoge atomistische Constitution hat auch der rhombische Libethenit, mit dem Unterschiede, dass $a b_{1}$ und $c_{1}$ nahe gleich sind, in Uebereinstimmung damit, dass die krystallostatischen Kräfte von $C u(=6 \cdot 2)$ und $P(=4 \cdot 3)$ gleich sind $(=12)$. Hiermit steht aucb die chemische Zusammensetzung des Libethenits in Uebereinstimmung, wenn man die Formel desselben $=2\left(\mathrm{Cu}_{2}[\mathrm{HO}] \mathrm{PO}_{4}\right)$ schreibt, wobei $4 \mathrm{Cu}=4 \mathrm{ab}$, $2 P=2 c$ und das Hydroxyl $(\mathrm{HO})_{2}$ als Kern- oder Centralmolekül in dem Atomcomplex betrachtet werden kann. Mit Ausnahme des Kernmolektils ist dieser Atomcomplex ähnlich dem des Andalusits (siebe Abth. III), mit welchem der Libethenit auch krystallographische Aehnlichkeit zeigt. Wie 
das Molekül des Markasits kann auch das Molekül des Libethenits als bestehend aus zwei monoklinen Partialmolekülen betrachlet werden, wodurch die monokline Anomalie ihrer Krystalle erklärt wird.

Für Tantalit sind die reciproken Werthe der Flächennormalen der zwei vorherrschenden Formen, des Prismas $\infty \breve{P} \frac{9}{4}$ und der Pyramide $P$ wie 2: 1, welches dem Verhältnisse der atomistischen Constanten für $\mathrm{Fe}(=6)$ und $T a$ resp. $N b(=12)$ entspricht; denn $N b$ ist $=4(3)+(2)+5 \cdot 8(2)=$ 94 und $T a=4(3)+(2)+12 \cdot 7(2)=182$. Hierbei ist zu bemerken, dass die Anzahl der Prismenflächen zu der der Pyramidenflächen sich wie die Anzahl der $\mathrm{Fe}$ - zu den $\mathrm{Ta}_{2}$ - und $\mathrm{Nb}_{2}$-Atomen verhält. Möglich ist jedoch, dass das Tantalat und das Niobat nicht nur verschiedene, zu molekularen Verbindungen vereinigte Moleküle constituiren, sondern zum Theil auch in demselben Molekule vorkommen kann, in welchem Falle dieses monoklinisch sein muss. In der That scheinen die Tantalitkrystalle aus Tammela wenigstens zum Theil monoklin zu sein, obgleich dieses wegen der unebenen Krystallflächen und der Undurchsichtigkeit der Krystalle nicht mit Bestimmtheit constatirt werden kann.

Bei dem Bary tocalcit scheint das Verhältniss zwischen Flächennormalen und Atomkräften weniger einfach zu sein, als bei den vorigen Mineralien, indem das Verhältniss der reciproken Werthe der Flächennormalen der Grundpyramide und des Orthopinakoids (=3:2) mit den alomistischen Constanten von $C$ und $C a$ (resp. $B a$ ) nur in dem Falle ubereinstimmt, dass man zu den der letzleren die Kerndynamide (2) mitrechnet, denn dann ist 12 (für $C$ ) : 8 (für $C a$ und $B a$ ) $=3: 2$. Doch kann man diese scheinbare Ausnahme auch so erklären, dass, während die Elementaratome der Moleküle der erstgenannten Mineralien eine gleiche Stellung (Kantenstellung) haben, sie in dem Grundmolekül des Barylocalcits eine verschiedene Stellung zu den resp. Flächennormalen einnehmen, nämlich das $C$-Atom eine Flächen-, $C a$ resp. $B a$ eine Kantenstellung, jenes also mit drei, dieses mit zwei Dynamiden angreifend (siehe hierüber weiter unten bei dem analog constituirten Pyroxen*).

Dass die reciproken Werthe der Normalen der Grundflächen als Ausdrücke für die molekularen Atomkräfte betrachtet werden können, zeig sich auch dadurch, dass, wenn man diese Werthe, wie es in der Statik geschieht, durch Linien veranschaulicht, und Kräfteparallelogramme resp. -Parallelipipeda mit diesen construirt, man aus jenen Grundkräften zu resultirenden Kräften kommen kann, durch die Diagonale ausgedrückt, welche

*) Bemerkt werden kann hier, dass diese Vergleichungen zwischen Atomkräften und den Normalen der Grundflächen nur als erster provisorischer Versuch zu betrachten sind. Doch scheint mir schon daraus hervorzugehen, dass eine Beziehung in dieser Hinsicht wirklich existirt und durch weitere vielseitigere Vorgleichungen näher begründet werden kann. 
zu den secundären Flächen normal stehen. Hierbei hat man zwei Hauptfälle zu unterscheiden, je nachdem die Grundfächen rechtwinklig gegen einander stehen (pinakoidale Ausbildung) oder schiefwinklig (prismatische Ausbildung). In jenem Falle kommt man, wenn man die Atomkräfte oder die reciproken Werthe der Normalen der pinakoidalen Grundflächen mil den Symbolen $a, b, c$ bezeichnet, durch fortgesetzte Zusammensetzungen zu denjenigen der secundären Flächen : $a b, a_{2} b, a_{3} b$ etc., $a b_{2}, a b_{3}$ etc. in der Zone $a-b, a c, a_{2} c, a_{3} c$ in der Zone $a-c$ etc. und weiter zu $a b c, a_{2} b c$, $a b_{2} c$ etc. in den pyramidalen Zonen; diese Flächenfolge ist die von Fraukenheim (Pogg. Ann. 1856, 97) sogenannte "normale (. Wenn aber die Prismenflächen Grundlächen sind, wie z. B. bei Orthoklas, Pyroxen, Amphibol, dann ist die Ausbildung prismatisch und die Flächenfolge » anomal «: $a b, a_{3} b, a_{5} b \ldots \ldots a b_{3}, a b_{5}$ etc., indem $a b+\bar{a} b=b_{2}, a b+b_{2}=a b_{3}, a b_{3}+$ $b_{2}=a b_{5}$ etc. Hierbei ist zu bemerken, dass, wie schon oben angedeutel worden ist, die physikalischen Molekule eine verschiedene Ausbildung haben können, als die von ihnen zusammengesetzten Krystallmoleküle, wie es z. B. mit dem Baryt der Fall zu sein scheint, welcher, obgleich sein physikalisches Grundmolekül nach obiger Deduction prismatisch ausgebildet ist, doch eine normale Flächenfolge in der Prismenzone zeigt.

Die genannten, den reciproken Werthen der Flächennormalen entsprechenden dynamischen Symbole, wie man sie bezeichnen könnte, sind in formeller Beziehung nichts anderes, als die Mill er'schen Indicesformeln mit zugesetzten Axenbezeichnungen und Weglassen des Axenzeichens, dessen Index $=0$. Wenn man die reciproken Werthe der Indices in diesen dynamischen Formeln nimmt, so kommt man zu den Normalensymbolen, welche den Parameterformeln mit Weglassung des Axenzeichens mit dem Parameter $=\infty$ gleich sind. Man könnte diese auch Molekularf ormeln nennen, da man auch zu ihnen kommt, wenn man sich die Molekule in den resp. Molekularrichtungen in gleicher oder ungleicher Anzahl verbunden denkt, so z. B. $a b^{1}, a b^{2}, a b^{3}$ etc., $a^{2} b, a^{3} b$ in der Zone $a-b$, $a c^{1}, a c^{2}, a c^{3}$ in $a-c$ u. s. w.; zum Unterschiede von den vorigen Formeln schreibe ich nämlich die Parameterzahlen rechts oben an die Buchstaben $a, b, c$, welche hier die resp. Hauplrichtungen bedeuten. Wie man die Normalen als Ausdrücke für die entsprechenden Flächen anwenden kann, so kann man auch diese Normalensymbole an Stelle der gewöhnlichen Parameterformeln anwenden, da sie für die allgemeiner vorkommenden Formen einfacher als diese sind, und ubrigens leicht in sie ubergefubrt werden können.

Wie man nun für die Grundmoleküle der Elemente oder ihrer Dynamidencomplexe drei, in Hinsicht ihrer gegenwärtigen Lage und Wirksamkeit verschiedene Arten von Dynamiden anzunehmen hat, nämlich 1) die formbildenden, nach aussen wirkenden Dynamiden, 2) die zwischen ihnen 
stehenden und sie verbindenden Dynamiden, und 3) die Kerndynamiden, von welchen die zwei letzteren bisweilen vermisst werden können, so haben wir auch in den von den Elementaratomen gebildeten Grundmolekulen der Silicale und übrigen Mineralien drei verschiedene Arten von Elementarbestandtheilen anzunehmen, nämlich die formbildenden positiven und indifferenten Elemente, die diese verbindenden Sauerstoffatome und schliesslich Kern- oder Centralatome rèsp. Moleküle, zu welchen letzteren vorzugsweise $\mathrm{Fl}$ resp. $\mathrm{Cl}$ geeignet scheinen, vielleicht auf Grund ihrer zur Verbindung der Atome und Moleküle geeigneten Eckendynamide (3), welche nach oben dargestellter Theorie auch in dem Sauerstoffatome und also auch in dem als Kernmolekül auftretenden Hydroxyl vorkommt. Hinsichtlich der Kernatome oder Kernmoleküle haben wir Moleküle resp. Molekularcomplexe oder zusammengesetzte Moleküle mit oder ohne Kernmoleküle zu unterscheiden.

Hier können wir nun schon elwas näher die anomalen Molekularzustände als beruhend auf einer kleinen Aenderung der gegenseiligen Stellung der Elementaratome annehmen, welches eine entsprechende Aenderung der auf diesen Atomstellungen beruhenden Richtungen der molekularen Kräfte, d. h. der Grundflächen des Krystalles zur Folge hat. - Man kann hierbei annehmen, dass die Atome und Molekule durch elektromagnetische Ströme mit einander verbunden sind, durch den elektrischen Gegensatz der positiven und negativen Dynamiden resp. Atome entstanden. Die Krystallmolekule können also als kleine Ampère'sche Solenoide betrachtet werden, indem die Krystallisationskraft als eine molekulare magnetische Kraft; und die chemische Affinität als eine elektrische Alomkraft bezeichnet werden kann. Aus den primären Strömen bilden sich durch Zusammensetzung intermediäre oder secundäre Ströme, und so entstehen neue Durchschnitlspunkte resp. Altraclionspunkte, wodurch die secundären Flächen gebildet werden.

\section{III.}

Wenn nun die in Abth. I und II dargestellten krystallographischen und chemischen theoretischen Sätze in der Hauptsache richtig sind, muss man durch ihre Zusammenstellung zu krystallochemischen Structur- oder Constitutionsformeln kommen können, welche den empirischen Formeln entsprechen. Es ist dies in der That der Fall, was in dem Folgenden gezeigt werden soll. Wir haben hierbei zuerst die Molekularverhältnisse der Silicate der- Feldspathgruppe zu betrachten und können dann von diesen ausgehend, oben (Abth. I) angeführter krystallographischer Molekulartheorie gemäss, zu denjenigen der übrigen Silicate gelangen.

Wie ich schon oben (Abth. II) angedeutet habe, zeigt der Orthoklas ein Verhältniss der reciproken Werthe der Normalen seiner Grundflächen 
$(001),(010),(T 01),(110),\left(1 T_{0}\right)$, das dem zwischen den molekularen Alomkräften entspricht. Dass man diese Flächen als Grundflachen oder Flächen der Grundform, welche den Richlungen der molekularen Attractionskräfte entsprechen, betrachten kann, zeigt sich theils aus ihrer Spaltbarkeil, theils aus ihrem allgemeinen Vorkommen. Von diesen Flächen sind die des Domas $(+\infty)$ und Prismas $(\infty P)$ in dieser Hinsicht einander ahnlich, indem sie keine oder nur unvollkommene Spaltbarkeit zeigen, und zusammen die einfachste Form der aufgewachsenen Adularkrystalle bilden. Ich betrachte sie demnach als den sechs $S i$-Atomen und die zwei übrigen Flächenpaare von $0 P$ und $\infty R \infty$ als den resp. $A l_{2}$ und $K a_{2}$ entsprechend; eine Annahme, wozu auch eine Vergleichung der krystallostatischen Atomzahlen von Silicium, Aluminium und Kalium (12:12:6) mit den reciproken Werthen der Normalen der genannten Flächen (110), $(\overline{1} 01),(001)$ und $(010)=2: 2: 1$ fubrt. Man kommt so zu nebenslehender krystallochemischer Structurformel fur diese Feldspathart.

In dieser krystallochemischen Structurformel des Orthoklases, dessen allgemeine Form der der Aetzfiguren auf der basischen Fläche ähnlich ist, verbinden die Sauerstoffatome die ubrigen mit ein-

Fig. 1.

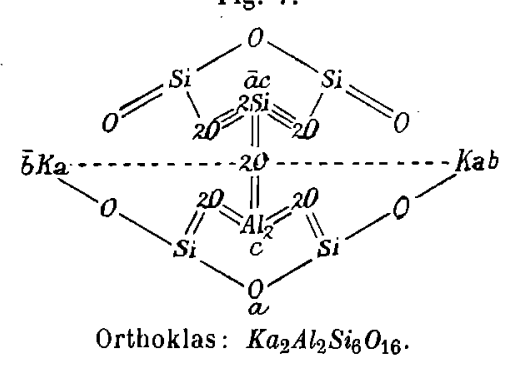
ander und verhalten sich also den indifferenten Dynamiden (2) in den elementaren Dynamidencomplexen ähnlich, indem sie keinen Einfluss auf die äussere Form des Molektlis ausuben, welche von den gegenseitigen Stellungen der oktaëdrischen $S i$-, $A l$ - und $K a$-Atome bewirkt wird. Wenn man nämlich in Uebereinstimmung mit oben angefuhrter Elementartheorie annimmt, dass die $S i$ - und $A l$-Atome mit ihren Kanten und die $K a$-Atome mit ihren Ecken gegen das Gentrum oder den Schwerpunkt des molekularen Kraftsystems gerichtet sind, können sie in solchen Stellungen zu einander gedacht werden, dass in der Zone der c-Axe (die hexagonale Zone) die SiOktaëder in hexagonaler, die $K a$-Oktaëder in annäherungsweise tetragonaler Stellung, in der Zone $b-c$ (die tetragonale Zone) die $K a$ - und $A l$-Atome in tetragonalen Stellungen und in der Zone der $b$-Axe (die rhombische Zone) die $A l$ - und $S i$-Atome in rhombischen oder rhombisch-monoklinen Stellungen sind.

Wie man also durch diese krystallochemische Molekularformel eine Erklärung der allgemeinen krystallographischen Verhältnisse des Orthoklases erhalten kann, so kommt man auch dadurch zu einer Erklärung der chemischen. Nach dieser Formel ist das halbe Orthoklasmolekül, dessen Form Fig. 3 ähnlich ist, durch den orthodiagonalen Durchschnitt in zwei Theile getheilt: $\mathrm{Ka}_{2} \mathrm{Al}_{2} \mathrm{Si}_{2} \mathrm{O}_{8}$ und $\mathrm{Si}_{4} \mathrm{O}_{8}$, welche nur durch $\mathrm{O}_{2}$ zusammen- 
gehalten werden. Eine Spaltung dieses Moleküls in seine zwei Theile muss also leicht vor sich gehen, was auch, wie die Erfahrung lehrt, der Fall ist bei den gewöhnlichsten Metamorphosen des Orthoklases in Muscovil $\left(\stackrel{\mathrm{H}}{2}_{2} \mathrm{Al}_{2} \mathrm{Si}_{2} \mathrm{O}_{8}\right)$ und $\mathrm{Kaolin}\left(\mathrm{H}_{2} \mathrm{Al}_{2} \mathrm{Si}_{2} \mathrm{O}_{8}+\mathrm{aq}\right)$, wobei $4 \mathrm{SiO}_{2}$ frei werden. Hierbei werden die $K a$-Atome, welche mil geringerer Kraft als die übrigen mit dem ganzen Complex zusammengehalten werden, zum Theil oder ganz durch andere gleichwerthige ersetzt. Auch das Verhältniss, dass die $b$ Fläche $(M)$ leichter durch Aetzungsmittel oder durch Verwitterung angegriffen wird als die $c$-Axe $(P)$, könnte aus dem verschiedenen chemischen Charakter der diesen Flächen entsprechenden Elemente erklärt werden.

Die $A l_{2}$-Atome und die $\mathbf{2} S i$-Atome in der Zone $a-c$ unterscheiden sich von einander nach obigen Annahmen der gegenseitigen molekularen Atomstellungen dadurch, dass die Aluminiumatome mit einer Fläche, die Siliciumatome mit einer Kante über, resp. neben einander stehen, doch so, dass in jedem Falle eine dodekaëdrische Symmetrieebene dieser Alome mit der Symmetrieebene des ganzen Moleküls zusammenfăllt. Eine geringe Veränderung dieser symmetrischen Molekularanordnung in eine schiefe muss dann eine Veränderung des normalen Orthoklases in einen anomalen triklinen Zustand (Anorthok las nach Rosenbusch) veranlassen. Solche Anorthoklase sind der von mir (diese Zeitschr. 8, 203) unter dem Namen $\mathrm{Ka} \mathrm{Na}$-Mikroklas bezeichnete Adularkrystall vom St. Gotthard, und weiler die von Förstner beobachteten anomalen $\mathrm{Na}$-Orthoklase von Pantellaria, welche zum Theil durch Temperaturerhöhung wieder in den normalen Zustand sich zurückführen lassen. Die Anorthoklase enthalten jedoch in der Regel mehr oder weniger $N a$, so dass man glauben könnte, dass dieser die trikline Anomalie bewirke. Bei Mikroklin muss man aber die trikline Form der schiefen Stellung des $A l$-Atoms allein zuschreiben, welche jedoch weniger schief als in den Albit- und Anorthitmolekülen ist, in welchen wahrscheinlich auch die $\mathrm{Na}$ - und $\mathrm{Ca}$-Oktaëder eine andere Stellung als das $K a$-Oktaëder des $K a$-Feldspaths einnehmen, nämlich eine Kantenstellung von ibren, von den letzteren verschiedenen Flächen- resp. Kerndynamiden bedingt; dadurch erklärt sich auch die Veränderung der Winkel in der Prismenzone.

Auf diesen verschiedenen Stellungen der Atome der $c-, \bar{a} c-$ und $b-$ Flächen kann man wohl auch annehmen, dass die verschiedenen Richtungen der optischen Elasticitätsflächen beruhen, indem die Auslöschungsrichlungen der resp. Flachen entweder parallel mit den Symmetrieebenen der diesen Flächen entsprechenden Elementaratome gehen, oder intermediäre, resullirende Stellungen zwischen diesen einnehmen. Ein näheres Eingehen auf diese Verhältnisse wurde jedoch hier zu weit führen.

In Uebereinstimmung mit den oben (Abth. I) angeführten Verhältnissen zwischen den Grundformen des Alkali- und Galcium-Feldspaths und der 
obigen Molekularformel des Orthoklases gemäss komml man zu folgender Molekularformel für Albit und Anorthit:

Fig. 2.

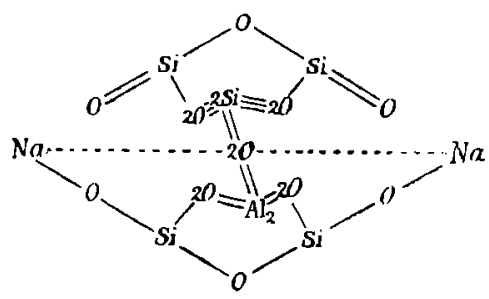

Albit : $\mathrm{Na}_{2} \mathrm{Al}_{2} \mathrm{Si}_{6} \mathrm{O}_{16}$. lig. 3 .

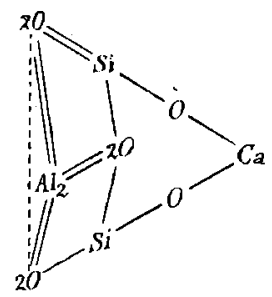

Anorthit: $\mathrm{CaAl}_{2} \mathrm{Si}_{2} \mathrm{O}_{8}$.

Das Molekularverbältniss zwischen Alkali- und Calciumfeldspath ist also wie $2: 1$, dem Atomverhälınisse $\stackrel{I}{R}_{2}: \stackrel{\text { II }}{C} a$ entsprechend. Dieses geht sowohl aus den oben (Abth. I) angefuhrten krystallographischen wie auch aus den chemischen und physikalischen Verhältnissen des Plagioklases hervor, wie folgende Zusammenstellung der Plagioklasarten zeigt, zu der ich durch eine Untersuchung einer grossen Menge, besonders finnischer Plagioklase gekommen bin.

\begin{tabular}{|c|c|c|c|c|}
\hline Albit & $\mathrm{Na}_{2} \mathrm{Al} l_{2} \mathrm{Si}_{6} \mathrm{O}_{16}=A b_{2}$ & $68,62 \%$ & 2,60 & $+20^{\circ}$ \\
\hline \multirow{3}{*}{ Oligoklas } & $A b_{6} A n_{1}=\left(N a_{2}, C a\right) A l_{2} S i_{5} O_{14}$ & 64,78 & 2,63 & +12 \\
\hline & $\left\{A b_{4} A n_{1}\right.$ & 63,26 & 2,64 & +9 \\
\hline & $A b_{3} A n_{1}$ & 61,90 & 2,65 & +4 \\
\hline Andesin & $A b_{2} A n_{1}=\left(N a_{2}, C a\right) A l_{2} S i_{4} O_{12}$ & 59,76 & 2,67 & -3 \\
\hline \multirow{4}{*}{ Labrador } & $A b_{2} A n_{2}$ & 55,56 & 2,69 & -16 \\
\hline & $A b_{2} A n_{3}=\left(C a, N a_{2}\right) A l_{2} S i_{3} O_{10}$ & 52,93 & 2,70 & -19 \\
\hline & $A b_{2} A n_{4}$ & 51,20 & 2,71 & -26 \\
\hline & $A b_{2} A n_{6}$ (Bytownit) & 49,10 & 2,73 & -29 \\
\hline Anorthit & $\mathrm{CaAl} l_{2} S i_{2} \mathrm{O}_{8}=A n_{1}$ & 43,07 & 2,76 & -36 \\
\hline
\end{tabular}

In rein chemischer Beziehung bilden die Plagioklasarten, wie auch andere grössere Silicatgruppen, homologe Reihen, denjenigen der organischen Verbindungen ähnlich, in krystallochemischer Hinsicht dagegen eine isomorphe Serie mit Andesin $=A b_{2} A n_{1}$ (nicht $A b_{2} A n_{2}$ oder $\left.A b A n\right)$ als Mittelglied; dieses geht daraus hervor, dass die Andesinvarietälen, welche sich zum Unterschiede von den gewöhnlich nicht kryslallisirten Oligoklas- und Labradorvarietäten oft durch deutliche Krystallform auszeichnen, diese Zusammensetzung haben, oder sich derselben nähern, so z, B. folgende : 


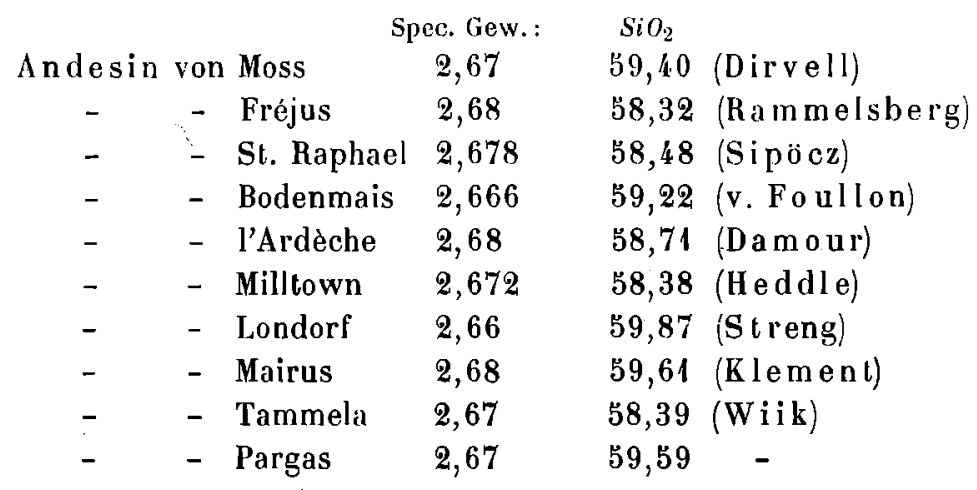

Dieselbe Stellung wie Andesin in $\operatorname{der} \mathrm{Na}, \mathrm{Ca}$-Plagioklasserie hat der Hyalophan $\left(\mathrm{Ka}_{2}, \mathrm{Ba}\right) \mathrm{Al}_{2} \mathrm{Si}_{4} \mathrm{O}_{12}$ in der $\mathrm{Ka}, \mathrm{Ba}$-Orthoklasserie, in welchem also auch die zwei Grenzglieder in dem Molekularverhältnisse 2: 1 zu einander stehen.

Dieses Molekularverhältniss (2:1), welches man in Uebereinstimmung mit dem analogen Alomverhältnisse $\left(\stackrel{I}{R}_{2}: \stackrel{\text { II }}{R}\right)$ als eine Aequivalenz zwischen 2 Mol. des Alkalisilicats und 1 Mol. des $C a$ - resp. Ba-Silicats betrachten kaon, ist nicht eine nur in der Feldspathgruppe vorkommende Erscheinung, sondern eine allgemeine, wie ich in dem Folgenden zeigen werde.

Ein ähnliches Verhältniss kommt nun auch zwischen dem alkalihaltigen Muscovit (resp. Paragonit) und dem Ca-fubrenden Margarit vor, wie folgende Molekularformeln zeigen, auf obige krystallographischen und krystallochemischen Beziehungen zwischen den Feldspath- und Glimmergruppen gegründet.

Fig. 4.

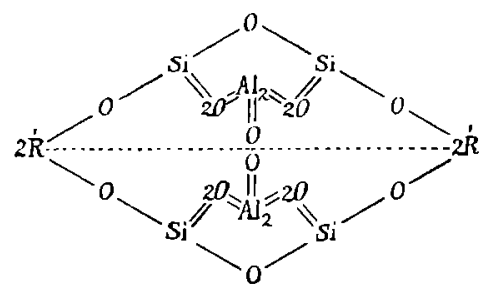

Muscovit : $2\left(\stackrel{\mathrm{r}}{R_{2}} A l_{2} S i_{2} O_{8}\right)$.
Fig. 5.

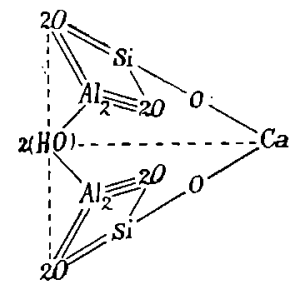

Margarit: $2\left[\left(\mathrm{H}_{2} \mathrm{Ca}\right) \mathrm{Al}_{2} \mathrm{SiO}_{6}\right]$.

Diesen Formeln gemäss kann man das Muscovitmolekul betrachten als bestehend aus zwei zu einem rhombischen oder quasi-rhombischen Krystallmolekül vereinigten monosymmetrischen physikalischen Molekülen, jedes dem halben Orthoklasmolekül $\left(\mathrm{Ka}_{2} \mathrm{Al}_{2} \mathrm{Si}_{2} \mathrm{O}_{8}\right)$ ähnlich, und das des Margarits aus zwei hemiëdrisch-triklinen Molekulen, dem halben Anorthitmolekül $\left(\mathrm{CaAl}_{2} \mathrm{SiO}_{6}\right)$ ähnlich, zu einem hemimorphen monoklinen Molekül vereinigt, welches mit den Aetzfiguren und den krystallographischen resp. 
krystalloptischen Eigenschaften des Muscovils, und so weit man es kennl, auch mit denen des Margarits in Uebereinstimmung steht.

Wenn man sich vorstellt, dass die $\mathrm{Al}_{2}$-Atome in dem Muscovitmolekuil dieselbe Stellung wie in dem des Orthoklases einnehmen, so enlsprechen ihre Oktaëderflächen der hasischen Fläche des Glimmers, und diese Doppelatome verbinden dann die drei Parlialmoleküle, welche man, wie oben (Abth. I) angefuhrt ist, im Muscovit anzunehmen hat, zu einem zusammengesetzten Tripelmolekul. Zu einer solchen Annahme kommt man auch in chemischer Hinsicht, denn da nach Tschermak (diese Zeitschr. 3, 22) im Muscovit (und Paragonit) $\mathrm{Ka}_{2}$ (resp. $\mathrm{Na}_{2}$ ) : $\mathrm{H}_{2}=1: 2$ ist, muss das Molekül dieser Glimmerarten $=3\left(\stackrel{I}{R_{2}} \mathrm{Al}_{2} S i_{2} \mathrm{O}_{8}\right)$ gesetat werden.

Wie nun die pseudorhombischen resp. monosymmetrischen Molekularformen des Muscovits und Margarits aus resp. denjenigen des Alkali- und Calciumfeldspaths hergeleitet werden können durch ihre Theilung in zwei Theile und eine zwillingsartige Verbindung des einen (vorderen) Theiles mit einem anderen desgleicben, mit der orthopinakoidalen $a$-Fläche als Zwillingsebene, so kommt man zu den Molekularformen der pseudorhomboëdrischen Glimmerarten der Biotitgruppe durch Verbindung dreier solcher Theile zu einem Drilling mit den prismatischen Flächen als Zusammenwachsungsebenen. Man kommt so zu folgenden krystallochemischen Structurformeln für Meroxen und Phlogopit, wobei zu bemerken ist, dass hier wie auch in einigen folgenden Formeln die Sauerstoffatome der Einfachheit wegen weggelassen sind.

Fig. 6.

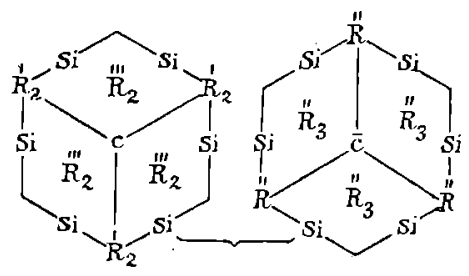

Meroxen: $\mathbf{3}\left(\stackrel{\mathrm{T}}{\mathrm{R}_{2}} \mathrm{Al}_{2} \mathrm{Si}_{2} \mathrm{O}_{8}+\underset{2 \mathrm{II}}{\mathrm{R}} \mathrm{SiO}_{2}\right)$
Fig. 7.

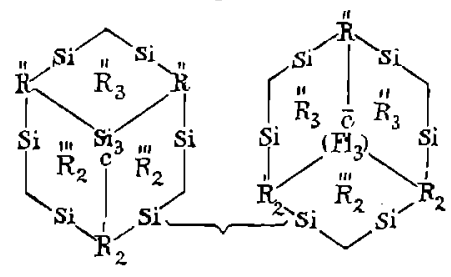

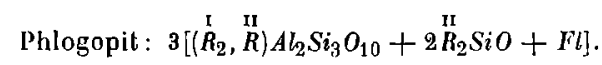

Die Molekularform des Meroxens kann als rhomboedrisch, die des Phlogopits als monoklinisch-rhomboëdrisch bezeichnet werden, in Uebereinstimmung mit den oben angefubrten Verhältnissen und den Aetzfiguren dieser Glimmerarten. Sie bestehen aus zwei in der Richtung der Hauptaxe mit einander vereinigten hemimorphen Partialmolekúlen, zwischen welchen in dem Phlogopit eine SiFl-Verbindung als Kernmolekúl anzunehmen ist. Diese zusammengesetzten Molekule sind ebenfalls hemimorph, mit den ${\stackrel{\text { II }}{R_{2}}}_{2}$-Atomen am einen, den äquivalenten $\stackrel{\text { II }}{R_{3}}$-Atomen am anderen 
Ende, eine Ilemimorphie, die auch bei den Krystallen des Biotits zuweilen constatirt werden kann.

Dass diese krystallochemischen Struclurformeln auch mit der chemischen Zusammensetzung übereinstimmen, zeigt folgende Zusammenstellung der aus ihnen berechneten Procentzahlen mit denen von T schermak (l. c. S. 135) mitgetheilten reducirten Analysen, wobei $\stackrel{\mathrm{III}}{R}=A l, \stackrel{\mathrm{II}}{R}=M g, \stackrel{1}{R}=K a$ gesetzl sind.

\begin{tabular}{|c|c|c|c|c|}
\hline \multirow{3}{*}{$4 \mathrm{SiO}_{2}$} & \multirow{2}{*}{\multicolumn{2}{|c|}{ Berechnet : }} & \multicolumn{2}{|c|}{ Meroxen: } \\
\hline & & & Vesuv: & Moravicza \\
\hline & \multicolumn{2}{|c|}{$4.0,27$} & 40,44 & 41,06 \\
\hline $\mathrm{Al}_{2} \mathrm{O}_{3}$ & \multicolumn{2}{|l|}{17,11} & 17,76 & 17,81 \\
\hline 4. $M g O$ & \multicolumn{2}{|l|}{26,85} & 27,96 & 29,08 \\
\hline \multirow{10}{*}{$\mathrm{K}_{2} \mathrm{O}$} & 15,77 & $\left(K a_{2} O+H_{2}\right.$ & 0) 13,92 & 12,05 \\
\hline & - & & $F l \quad 0,92$ & 一 \\
\hline & 100,00 & & 100,00 & 100,00 \\
\hline & \multicolumn{2}{|r|}{ Berechnet: } & \multicolumn{2}{|c|}{$\begin{array}{c}\text { Phlogopit. } \\
\text { Pargas: }\end{array}$} \\
\hline & $15 \mathrm{SiO}_{2}$ & 45,59 & \multicolumn{2}{|c|}{44,03} \\
\hline & $3 \mathrm{Al}_{2} \mathrm{O}_{3}$ & 15,65 & \multicolumn{2}{|c|}{14,05} \\
\hline & $13 \mathrm{MgO}$ & 26,34 & \multicolumn{2}{|c|}{28,36} \\
\hline & $2 \mathrm{Ka}_{2} \mathrm{O}$ & 9, 53 & \multicolumn{2}{|c|}{10,17} \\
\hline & \multirow[t]{2}{*}{$3 F l$} & 2,89 & \multicolumn{2}{|c|}{4,27} \\
\hline & & 100,00 & \multicolumn{2}{|c|}{100,88} \\
\hline
\end{tabular}

Diese Uebereinstimmung scheint genúgend zu sein, wenn man die ausserordentliche Complication der Zusammensetzung dieser Glimmerarten in Betracht zieht. Die Molekularformeln 6 und 7 stellen die Grundmolektile des Biotits nur in ihren einfachsten Formen dar, denn hier wie bei den einfachsten Muscoviten haben wir aus oben angefuhrten Gründen drei solche, zu einem Tripelmolekül in der Richtung der Hauptaxe durch die äquivalenten ${\stackrel{\mathrm{III}}{R_{2}}}_{2}$ und $\stackrel{\mathrm{II}}{R_{3}}$ vereinigte Moleküle anzunehmen. Dazu kommt noch, dass die Meroxen- und Phlogopitmoleküle höchstwabrscheinlich mit einander Molekularverbindungen eingehen können, wodurch der Uebergang zwischen dem optisch-einaxigen oder nahe einaxigen Meroxen und dem. deutlich optisch-zweiaxigen Phlogopit erklärt werden kann. Eine solche Molekularverbindung zwischen Phlogopit und Muscovit hildet wahrscheinlich die von Tschermak als A nom it bezeichnete Abart des Phlogopits.

In oben genannten Molekularformen des Muscovits und Biotits entsprechen die Partialformen wie gesagt dem einen, vorderen Theile des Orthoklasmoleküls; in der Molekularform des Lith ionglimmers (Lepidolith und Zinnwaldit) mussen sie in Hinsicht auf deren asymmetrische Aetzfiguren als dem vorderen Theile des Albits ähnlich angenommen werden. Von 
diesen gehört der Lepidolith der homologen Reihe des Muscovits an $\left(\stackrel{I}{R}_{2} \mathrm{Al}_{2} S i_{n} O_{2 n+4}\right.$ mit $n=1$ für Margarit, $n=2$ für Muscovit, $n=3$ für Lepidolith), während der Zinnwaldit (Lithionil) sich dem Biotit (Phlogopil) in krystallochemischer Hinsicht anschliesst. Für Lepidolith mit normalsymmetrischer oplischer Axenebene haben wir eine ähnliche pseudorhombische Molekularform wie die des Muscovits, und fưr Zinnwaldit mit parallelsymmetrischer Axenebene eine der des Biotits ähnliche anzunehmen, aber in vier resp. sechs hemiëdrisch-trikline Partialformen getheilt, durch ein Kernmolekül ähnlich dem bei Phlogopit zusammengehalten. Im Zusammenhang hiermit kann bemerkt werden, dass die oben angeführte Molekularform (5) des Margarits, wenn man sie in ein in der $b$-Axe verdoppelles holomorphes Krystallmolekül verwandelt, 4 Mol. Hydroxyl als Kern- oder Centralmolekül enthält.

In Uebereinstimmung mit der Herleitung der Molekularformen des Biotits aus derjenigen des Alkalifeldspaths resp. Alkaliglimmers haben wir die des alkalifreien Sprödg limmers aus der hemiëdrisch-triklinen Molekularform des Anorthits resp. der hemimorph - monoklinen des Margarits herzuleiten, und kommen dann zu einem mimetisch-hexagonaleu Formencomplex in verwendeter Stellung zu der des Biotits, mit den Si-Atomen in den zwei hemimorphen Partialcomplexen nur den drei abwechselnden Prismenflächen entsprechend und die drei $A l_{2}$ - resp. $M g_{3}$-Atome den drei trigonalen Trapezoëder- resp. Pyramidenflächen, also zu dem trapezoëdrischletartoëdrischen Formentypus des Glimmers (siehe oben Abth. I), folgender empirischen Formel gemäss :

$$
3\left(\mathrm{CaAl}_{2} \mathrm{SiO}_{6}+\mathrm{Mg}_{2} \mathrm{SiO}_{5}+\mathrm{H}_{2} \mathrm{Al}_{2} \mathrm{O}_{4}\right) \text {. }
$$

Diese Formel entspricht der Zusammensetzung des Sprödglimmers (Seybertit, Brandisit), wie eine Zusammenstellung der daraus berechneten Procentzahlen mit den für diese Glimmerarten empirisch gefundenen Zahlen zeigt. Das Partialmolekul $3\left(\mathrm{H}_{2} \mathrm{Al}_{2} \mathrm{O}_{4}\right)$ kann als Kernmolekül zwischen den zwei anderen hemimorphen Partialmolekulen betrachtet werden, wodurch das Molekul des Sprödglimmers sich dem des Chlorits anschliesst.

Bei allen diesen Deductionen der Molekularformen der Glimmerarten aus denen der Feldspatharten ist nur der eine Theil $\left(\stackrel{\mathrm{r}}{R}_{2} \mathrm{Al}_{2} \mathrm{Si}_{2} \mathrm{O}_{8}\right)$ von diesen letzteren betheiligt; der andere aus $4 \mathrm{SiO}_{2}$ bestehende Theil der Molekularform des Albits kann auf ähnliche Weise behandelt werden, und dann kommt man zu analogen Zwillingscomplexen, welche mit den Grundformen der drei $\mathrm{SiO}_{2}$-Modificationen, des rhombischen oder rhombisch-triklinen Asmanit, des hexagonalen, bei gewöhnlicher Temperatur triklinen Tridymit und des trapezoëdrisch-tetartoëdrischen $Q \mathbf{u}$ a r $z$, Uebereinstimmung zeigen.

Was besonders den Quarz betrifft, so kann angefuhrt werden, dass 
die genannten zu einem pseudohexagonalen Complex vereinigten triklinen P'artialformen des Albits je nach ihrer gegenseitigen Stellung ihre (10T)liächen nach links oder rechts geneigt haben und also eine schraubenförmige Anordnung bilden, der spiralförmigen Anordnung der Moleküle entsprechend, welche man a priori bei dem circularpolarisirenden Quarz anzunehmen hat. In Uebereinstimmung hiermit mussen auch die elektromagnelischen Ströme, welche man in gewöhnlichen Füllen in einer Ebene um die Krystallmoleküle kreisend anzunehmen hat, hier auch spiralförmig angeordnet sein, und also auf den intramolekularen optischen Aether auf dieselbe Weise wirken, wie nach Faraday's Versuch ein elektromagnetischer Schraubendraht auf einen polarisirten Lichtstrahl.

Wie oben angedeutet ist, kann man den dodekaédrischen Sodalithkrystall betrachten als zusammengesetzt aus vier mit einander zwillingsartig vereinigten hexagonalen Molekularformen des Nephelins; auf ähnliche Weise kann auch das Krystallmolekül des Granats zum Theil aus acht rhomboëdrischen hemimorphen Partialmolekulen des ihm ähnlich zusammengeselzlen Biotils gebildet werden, besonders die Granatvarielät, welche in den von mir beobachteten durch Granit metamorphosirten Gneissfragmenten vorkommt. Wie nun der Sodalith sich zum Nephelin und der Granal zu Biolit verhält, so verhalten sich wahrscheinlich auch die regulären oder anomal regulären Melan ophlogit und $\mathrm{Cr}$ istobalit zu den hexagonalen Modificationen der Kieselsäure, wobei der geringe Schwefelgehalt $\left(\mathrm{SiS}_{2}\right.$ nach Streng) in dem erstgenannten als Centralmolekúl in dem regulären Molekularcomplex angenommen werden kann, in Uebereinstimmung mit dem Schwefelmangan $(M n S)$ in dem letraëdrisch-regulären Helvin. Auch in dem Sodalithmolekul hat man einen Kernmolekularcomplex von $\mathrm{NaCl}$ Molekülen und in dem des Haüyns, Noseans, Lasurits von $\mathrm{CaSO}_{4}$ und $\mathrm{Na}_{2} \mathrm{SO}_{4}$ resp. $\mathrm{NaS}$ anzunehmen, von acht hemimorphen nephelinartigen Molekülen umschlossen.

Im Anschluss an die Silicate der Glimmergruppe mögen hier auch die der Chloritgruppe betrachtet werden, nämlich :

$$
\begin{aligned}
& \text { Prochlorit } \quad \stackrel{\mathrm{u}}{\mathrm{R}} \stackrel{\mathrm{II}}{\mathrm{R}} \mathrm{Si}_{2} \mathrm{O}_{8}+\mathrm{H}_{6} \mathrm{Al}_{2} \mathrm{O}_{6} \text {, } \\
& \left.\begin{array}{l}
\text { Klinochlor } \\
\text { Pennin }
\end{array}\right\} 2 \mathrm{H}_{2} \mathrm{II}_{3} \mathrm{Si}_{2} \mathrm{O}_{8}+\mathrm{H}_{4} \mathrm{Al}_{2} \mathrm{O}_{5} \text {. }
\end{aligned}
$$

Diese Formeln slehen in Uebereinstimmung mit der Ansicht von R a mmelsberg (Mineralchemie 1875, 487), dass die Chlorite Aluminiumbydroxyd enthalten, und mit der von Tschermak (Die Chloritgruppe II), nach welcher ein serpentinartiges Glied in ihre Zusammensetzung eingeht. Dass diese Formeln den Analysen entsprechen, zeigt folgende Znsammenstellung; hierbei $\operatorname{sind} \stackrel{\text { II }}{R}$ im Prochlorit $=\stackrel{\text { II }}{F} e$ und $\stackrel{\text { II }}{R_{3}}=M g_{3}$ gesetzt, wodurch die $\mathrm{Zu}$ sammensetzung am nächsten dem eisenarmen Chlorit (Lophoit Breit- 
ha $\mathrm{upt}$ ) entspricht. In dem eisenreicheren Chlorit ( $O \mathrm{nk}$ o it Breith.) sind wohl die Lophoilmeleküle zum Theil durch Moleküle mit $\stackrel{\text { II }}{R_{3}}=\stackrel{\text { II }}{F} e_{3}$ substituirt; ebenso ist im Klinochor ein Theil von $\stackrel{\mathrm{II}}{R}_{3}=F e_{3}$ zu setzen.

\begin{tabular}{cccc} 
& Berechnet: & \multicolumn{2}{c}{ Prochlorit } \\
$2 \mathrm{SiO}_{2}$ & 25,64 & 27,32 & 26,51 \\
$\mathrm{Al}_{2} \mathrm{O}_{3}$ & 21,79 & 20,69 & 21,81 \\
$3 \mathrm{MgO}$ & 25,64 & 24,89 & 22,83 \\
$\mathrm{FeO}$ & 15,39 & 15,23 & 15,00 \\
$3 \mathrm{H}_{2} \mathrm{O}$ & 11,54 & 12,00 & 12,00 \\
\cline { 2 - 4 } & 100,00 & 100,13 & 98,15 \\
& & $\mathrm{~K} 1 \mathrm{inochlor}:$ \\
$4 \mathrm{SiO}_{2}$ & 36,70 & $30-37 \%$ \\
$\mathrm{Al}_{2} \mathrm{O}_{3}$ & 15,60 & $13-18$ \\
$6 \mathrm{MgO}^{\mathrm{O} O}$ & 36,70 & $31-37$ \\
$4 \mathrm{H}_{2} \mathrm{O}$ & 11,00 & $9-13$ \\
& 100,00 & \multicolumn{2}{c}{9}
\end{tabular}

Von diesen empirischen Formeln kommt man zu krystallochemischen, rhomboëdrischen resp. rhomboëdrisch-monoklinen Structurformeln, von welchen die des Prochlorits sich an die des Meroxens, die für Klinochlor an die des Phlogopits anschliesst, den Aetzfiguren und Krystallformen der resp. Ghloritarten gemåss. Wenn man nämlich obige Formel für Prochlorit verdreifacht, kommt man zu einem hemimorphen Molekularcomplex, und wenn man diesen verdoppelt, zu einem holomorphen $=6\left(\mathrm{RM}_{3} \mathrm{Si}_{2} \mathrm{O}_{8}+\mathrm{H}_{2} \mathrm{O}\right)$, dessen zwei hemimorphe Theile mit dem einen, $\stackrel{\mathrm{R}}{3}_{3}$ enthaltenden Theile des Meroxenmoleküls übereinstimmen, und ein pseudorbomboëdrisches Hydrargillitmolekül als Kernmolekúl umschliessen. Die $\mathrm{H}_{2} \mathrm{O}$-Moleküle sind zum Theil als Krystallwassermoleküle symmetrisch um die drei Partialmolekuile angeordnet zu betrachten, theils als Kernmolekule von Hydroxyl innerhalb derselben. - Diese Verbindung eines optisch negativen glimmerartigen und eines optisch posiliven hydrargillitartigen Molekuls erklärt die schwache Doppelbrechung und den theils optisch positiven, theils negativen Charakter des Chlorits. Doch mag hier bemerkt werden, dass die variable Zusammensetzung des Chlorits zur Annahme von noch einer mit der obengenannten homologen Verbindung fưr Prochlorit fübrt: $R_{R_{3}} \mathrm{Si}_{3} \mathrm{O}_{10}+\mathrm{H}_{6} \mathrm{Al}_{2} \mathrm{O}_{6}$, in welchem Falle das Centralmolekul in genanntem Molekularcomplex von drei kaolinarligen Partialmolekúlen zusammengesetzt gedacht werden kann.

Fur Klinochlor kommt man von oben angeführter Formel zur Annahme eines kaolinartigen monosymmetrischen Partialmoleküls $\left(\mathrm{H}_{2} \mathrm{Al}_{2} \mathrm{Si}_{2} \mathrm{O}_{8}+\mathrm{H}_{2} \mathrm{O}\right)$ mit zwei serpentinartigen asymmetrischen, $2\left(\mathrm{H}_{2} \stackrel{\mathrm{MI}}{\mathrm{M}} \mathrm{SiO}_{3} \mathrm{~S}_{6}\right)$, zu einem rbomboëdrisch-monasymmetrischen Complex vereinigt. Wenn man drei solche 
so mit einander nach den prismatischen, den $S i$-Atomen entsprechenden Wlächen richtungen drillingsartig verbunden denkt, dass die drei kaolinartigen Partialmoleküle nach innen, die serpentinartigen nach aussen steheo, so kommt man zu einem rhombëdrischen Molekularcomplex, im Ganzen ana$\log$ mit dem von Prochlorit, aber in seinen einzelnen Theilen und also auch der Zusammensetzung nach mit dem Klinochlor ubereinstimmend, d. h. zu dem Grundmolekul des Pennins, welches die chemische Zusammensetzung des Klinochlors mit der hexagonalen Form des Prochlorits vereinigt. Hierbei kann man annehmen, dass entweder alle in einen Penninkrystall eingehenden Molekule von dieser Beschaffenheit sind, oder dass nur ein Centralmolekiul eine solche $Z$ willingsstructur hat, und dass an dieses einzelne sich Klinochlormolekule symmetrisch nach den Hauptrichtungen anschliessen, so dass also der Krystall im Ganzen eine ähnliche Structur wie die des Centralmoleküls erhält. So erklärt sich die von Tschermak angezeigte verschiedene Beschaffenheit des Pennins von Zermatt und des Pennins vom Zillerthal und Ala, welche letzteren die äussere Form des Pennins mit der inneren Structur des Klinochlors vereinigen.

Von den von Tschermak sogen. Leptochloriten mag hier nur der in deutlichen rhomboëdrisch-hemimorphen Krystallen auftretende Cronstedtit betrachtet werden. Wenn man in Uebereinstimmung mit der sehr vollkommenen basischen Spaltbarkeit das gewöhnlich als $3 R$ bezeichnete Rhomboëder als Grundrhonboëder $(=R)$ annimmt, so wird $c=3$,44 oder annähernd gleich der Hauplaxe des Pennins. Auch erhält man für den Cronstedit eine Molekularformel ähnlich denen des Biotits und Chlorits, wenn man die von Janowsky gegebene empirische Formel auf folgende Weise schreibt:

$$
3 \stackrel{\mathrm{II}}{\mathrm{Fe}} \mathrm{Fi}_{3} \mathrm{Fi}_{2} \mathrm{Si}_{2} \mathrm{O}_{10}+12 \mathrm{H}_{2} \mathrm{O} \text {. }
$$

Diese fuhrt nämlich zur Annabme eines rhomboëdrischen Molekularcomplexes mit den sechs $\mathrm{Si}$-Atomen an den Seiten, ${ }^{\mathrm{III}} \mathrm{Fe}_{2}$ den drei Rhomboëderflächen des einen und $3 \mathrm{Fe}_{2}$ denen des anderen Endes entsprechend, und mit den $12 \mathrm{H}_{2} \mathrm{O}$-Molekulen symmetrisch um diesen Complex angeordnet. Als Zwischenglieder $\mathrm{zwischen}$ Ghlorit und Cronstedtit, und zum Theil vielleich als isomorphe Verbindungen von ihnen, können Delessit und der daran sich anschliessende, von mir beobachtete Euralit betrachtet werden.

Durch die Chloritgruppe wird der Uebergang von der Glimmergruppe zu den Talk- und Ka olingruppen vermittelt. Diese bilden in chemischer Hinsicht zwei analoge homologe Reihen, von welchen die zwei Hauptrepräsentanten sich in Uebereinstimmung mit den bisher angeführten Silicaten durch folgende krystallochemische Formeln ausdrutcken lassen: 
Fig. 8 .

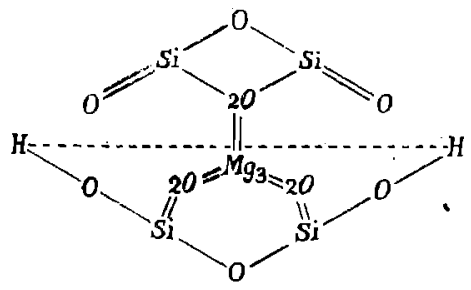

Talk : $\mathrm{H}_{2} \mathrm{Mg}_{3} \mathrm{Si}_{4} \mathrm{O}_{12}$.
Fig. 9.

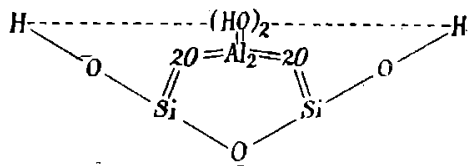

Kaolin : $\mathrm{H}_{2} \mathrm{Al}_{2} \mathrm{Si}_{2} \mathrm{O}_{8}+\mathrm{H}_{2} \mathrm{O}$.

Wenn man in der Molekularformel des Talks $M g_{3}$ durch $A l_{2}$ und in der des Kaolins $\boldsymbol{A l}_{2}$ durch $\boldsymbol{M} g_{3}$ ersetzt, kommt man zu den Formeln für resp. Pyrophyllit und Serpentin. Diesen Formeln gemäss kann die Molekularform des Talks als rhombisch-monoklin, die des Kaolins als hemiedrisch-monosymmetrisch bezeichnet werden; jene besteht wie die des Orthoklases aus zwei ungleichen Theilen, diese nur aus dem einen dieser Theile, welcher verdoppelt werden kann, wodurch ein Muscovit-ähnliches, quasi-rhombisches Krystallmolekul entsteht, mit Hydroxyl als Centralmolekul.

Diese Molekularformeln stehen in Uebereinstimmung mit den krystallographischen und optischen Verhältnissen dieser Silicate, so weit sie bekannt sind, und lassen auch eine einfache Erklärung der Talk- und Kaolinmetamorphosen zu, wie noch weiter im Folgenden gezeigt werden soll. Was besonders den Kaolin betrifft, so zeigt das von M i ers gefundene Axenverhältniss $a: b: c$ $=0,5748: 1: 1,5997 ; \beta=83^{0} 11^{\prime}$, dass der Kaolin in Uebereinstimmung mit dem Muscovit eine dreimal so grosse Hauptaxe wie die des Orthoklases hat, was man findet, wenn man die Grundformen der drei Silicate auf ein rechtwinkliges Axenverhältniss ähnlich dem für Muscovit (nach v. Kokscharow) zurückführt; dieses führt auch für den Kaolin zur Annahme eines Tripelmolekuls in der Richtung der Hauptaxe, ähnlich dem für Muscovit. Dass dieses Grundmolekúl des Kaolins als hemiëdrisch bezeichnet werden kann, zeigen die von A. Dick und Miers (Mineral. Magazine 1888; siehe diese Zeitschr. 17, 523) beobachteten Kaolinkrystalle von Anglesea, welche Prismen- und Pyramidenflächen ohne parallele Gegenflächen haben, in Analogie mit den von Williams angefuhrten Pyroxenkrystallen (On the possibility of Hemiëdrism in the Monoclinic Crystal System, Amer. Journ. of Sc. 38; diese Zeitschr. 19, 646).

Da nun nach obiger Auffassung die blättrige Textur und sehr vollkommene Spaltbarkeit der genannten glimmerartigen Silicate (Phyllite) dadurch bedingt sind, dass ihre Molektule aus zwei oder drei einzelnen Molekülen in der Richtung der Hauptaxe zusammengesetzt sind, so folgt daraus, dass in den dichten, erdigen oder z.Th. amorphen Varietäten dieser Silicate (Sericit, z. Th. Steatit, Agalmatolith etc.) die Moleküle zum grössten Theile in ibre einzelnen 
Theile gesondert werden, wodurch ihre Aehnlichkeit in chemischer, aber grosse Unähnlichkeit in physikalischer Hinsicht mit den krystallisirten Varieläten erklärt wird. So kann man z. B. annehmen, dass die Moleküle des Muscovits in zwei oder drei verschiedene einzelne Arten von Molekülen, $\mathrm{Ka}_{2} \mathrm{Al}_{2} \mathrm{Si}_{2} \mathrm{O}_{\mathrm{s}}$ resp. $\mathrm{Na}_{2} \mathrm{Al}_{2} \mathrm{Si}_{2} \mathrm{O}_{8}$ und $\mathrm{H}_{2} \mathrm{Al}_{2} \mathrm{Si}_{2} \mathrm{O}_{8}$ zerfallen, von welchen die ersteren sich zu den Molekülen des Eläoliths wie die des Muscovits zu den des Biotits verhalten. Aus solchen Molekülen bestehen wahrscheinlich die unter den Namen Gieseckit, Liebenerit, Pinit, Pyrargillit etc. bekannten Metamorphosen des Eläoliths und Cordierits, da in diesen nur zum kleineren Theile wirkliche, deutliche Glimmerblättchen vorkommen.

Wie man aus den oben (Abth. I) angeführten krystallographischen Verhältnissen des Cordierits und des T u rmalins zu einander und zu der Feldspathgruppe (besonders dem Anorthit) sehliessen kann, haben wir fưr die Silicate ähnliche Molekularformen (10 und 11) anzunehmen wie die für Muscovit und Biotit, nämlich rhombische und rhomboëdrische, jede mit Kernmolekülen.

Fig. 10.

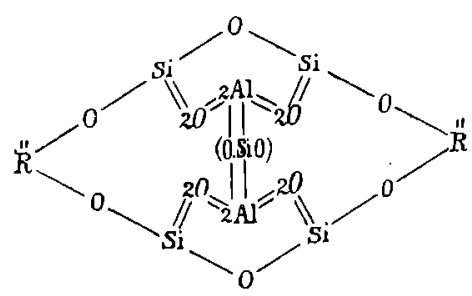

Cordierit: $2\left(\stackrel{\mathrm{II}}{\mathrm{RA}} \mathrm{l}_{2} \mathrm{Si}_{2} \mathrm{O}_{8}\right)+\mathrm{SiO}_{2}$.
Fig. 11.

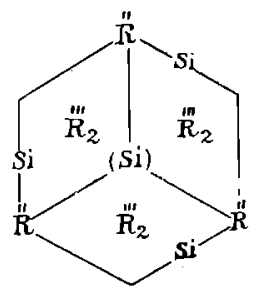

Turmalin : $3\left(\stackrel{\text { II II }}{\mathrm{R}_{2}} \mathrm{SiO}_{\mathrm{G}}\right)+\mathrm{SiO}_{2}$

Das Cordieritmolekül ist, da es ein zweiatomiges Radical enthält, zu betrachten als zusammengesetzt aus zwei mit dem Margaritmolekül analogen hemimorphen Molekülen $2\left(\stackrel{\mathrm{II}}{\mathrm{R}} \mathrm{Al}_{2} \mathrm{Si}_{2} \mathrm{O}_{8}\right)$ von (SiO) zusammengehalten, d. h. eine Verbindung, die sich zur Kieselsäure wie Hydroxyl $(H O)$ zu Wasser verhält. Dass ein solches hemimorphes Cordieritmolekül, das sich zu dem holomorphen wie das Anorthit- zu dem Albitmolekül verhält, wirklich vorkommt, zeigen die Analysen, deren $\mathrm{SiO}_{2}$-Gehalt zwischen 50,65 und 43,60\% wechselt, und meistentheils gleich $48-49 \%$ ist. Diese Zahlen stimmen nämlich mit den aus den genannten Silicaten $\left(\mathrm{Mg}_{2} \mathrm{Al}_{4} \mathrm{Si}_{5} \mathrm{O}_{18}\right.$ und $\left.\mathrm{MgAl}_{2} \mathrm{Si}_{2} \mathrm{O}_{8}\right)$ berechneten Procentzahlen $\left(\mathrm{SiO}_{2}=\mathbf{5 1 , 3 7}\right.$ und $45,80 \%$ uberein, und vorzugsweise mit ihrer intermediären mit Andesin analogen Verbindung, deren $\mathrm{SiO}_{2}$-Procent $=48,74$ ist.

Die Aehnlichkeit der Molekularformen 10 und 11 mit denjenigen des Glimmers erklärt die Pseudomorphosen des Muscovits und Biotits nach Cordierit und Turmalin, welche z. B. bei Torro in Tammela (Finnland) vorkommen. 
Bei der Cordieritmetamorphose geht, wie bei der Metamorphose des Orthoklases, das dem längsten Molekularabstand (b) entsprechende $M g$ resp. $\mathrm{Fe}$ fort, und wird durch $\mathrm{Ka} \mathrm{a}_{2}$ resp. $\mathrm{H}_{2}$ ersetzt, wobei die oben genannten Zwischenglieder zwischen Cordierit und Muscovit entsteben (Pyrargyllit, Fahlunit, Pinit etc.), welche als rhombische Alkali-Aluminiumsilicate sich zum Nephelin wie Cordierit zum Turmalin verhaltend betrachtet werden können.

Doch scheint auch die Cordieritsubstanz selbst eine hexagonale Modification zu baben; als solche möchte ich nämlich die als Drillingscomplexe von rhombischen Cordieriten angesehenen, mimetisch-hexagonalen Krystalle im Andesit von Gabo de Gata u. a. vulkanischen Gesteinen betrachten. Da nämlich wirkliche $\mathrm{Zwillinge} \mathrm{von} \mathrm{Gordierit} \mathrm{ziemlich} \mathrm{selten} \mathrm{sind,} \mathrm{und} \mathrm{da} \mathrm{diese}$ Krystalle in Hinsicht ihrer Structur mit anderen bei gewöhnlicher Temperatur anomalen Krystallen, z. B. Milarit, Aehnlichkeit zeigen, so scheinen sie mir auch auf ähnliche Weise betrachtet werden zu können, nämlich als bei ihrer Bildungstemperatur wirklich normal hexagonal, aber bei niederer Temperatur sich in rhombische Theile sondernd. Denkt man sich die rhombischen Zwillingsindividuen als von dem Doma $\{011\} \breve{P}_{\infty}$ begrenzt mit dem Winkel $58^{0}$ 22' $^{\prime}$, so erhalten die Krystalle Aehnlichkeit mit Beryllkrystallen, welche bei gewöhnlicher Temperatur in anomalem Zustande als aus rhombischen Theilen bestehend betrachtet werden können. Die Molekularform (11) des Turmalins schliesst sich am nächsten der des Sprödglimmers an, indem sie aus asymmetrischen Partialmolekulen zu einem tetartoëdrischhexagonalen Complex verbunden bestebt, doch nicht trapezoëdrisch-, sondern rhomboëdrisch-telartoëdrisch in Uebereinstimmung mit der von Jerofej ew, Solly, Ram say u. A. beschriebenen rhomboëdrisch-tetartoëdrischen Ausbildung und den asymmetrischen Aetzfiguren des Turmalins.

Diese Molekularform entspricht jedoch nur der Zusammensetzung eines Theiles des Turmalins, nämlich den alkalifreien oder alkaliarmen MagnesiumEisen-Turmalinen, welche nach $R$ ammelsberg durch die empirische

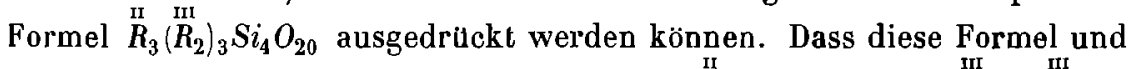
also auch die Molekularformel (11), wenn man $3 R=F e+2 M g, 3 R_{2}={ }_{B_{2}}^{\text {III }}+$ $2 \mathrm{III}_{2}$ setzt, mit den Analysen ubereinstimmen, zeigt folgende Zusammenstellung.

Berechnel:

\begin{tabular}{cr}
$4 \mathrm{SiO}_{2}$ & 35,95 \\
$2 \mathrm{Al}_{2} \mathrm{O}_{3}$ & 30,80 \\
$\mathrm{~B}_{2} \mathrm{O}_{3}$ & 10,47 \\
$\mathrm{FeO}$ & 10,79 \\
$2 \mathrm{MgO}$ & 11,98 \\
\cline { 2 - 2 } & 100,00
\end{tabular}

Tu rmal in aus Haddam

nach Riggs: nach Rammelsberg:

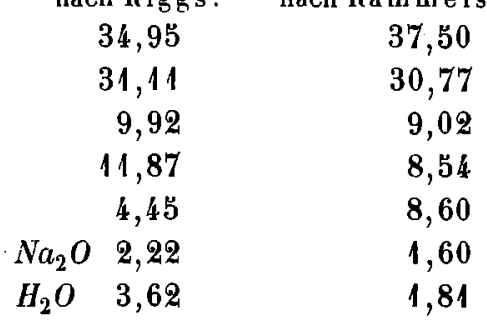


Wenn man sich $M_{g}$ zum Theil durch $\mathrm{Na}_{2}$ und $\mathrm{H}_{2}$ substituirt denkl, so unterscheiden sich die berechneten Procentzahlen nicht mehr von denen der zwei Analysen, als diese von einander. Diese Analysen zeigen die grosse Variation des Turmalins auch von demselben Fundorle an. In der That zeigt der Turmalin in Hinsicht seiner Zusammensetzung eine ähnliche Mannigfaltigkeit wie die Glimmerarten, was durch Verbindung von qualitaliv verschiedenen, aber quantitativ ähnlichen Molekularcomplexen theils in paralleler, theils in molekularer Zwillingsstellung erklärt werden kann.

Zu diesen alkalifreien Molekularcomplexen kommen nun weiter die des lichtgefärbten Alkaliturmalins, welcher der von Rammelsberg aufgestellten empirischen Formel gemäss sich durch folgende Structurformel ausdrücken lässt:

$$
\left(3 \stackrel{\mathrm{I}}{R}_{2}\right)\left(B_{2} 3 A l_{2}\right) S i_{6} O_{27}+\left(B_{2} 3 A l_{2}\right) S i_{3} O_{18} \text {. }
$$

Nach dieser Structurformel besteht der Alkaliturmalin aus zwei verschiedenen hemimorphen Molekularcomplexen, der eine alkalihaltig mit $6 \mathrm{Si}$-Atomen, also hemiëdrisch, der andere alkalifrei mit $3 \mathrm{Si}$-Atomen oder tetartoëdrisch, ähnlich der des $M g$-Turmalins. Die alkalihaltigen, rhomboëdrischen Molekularcomplexe verhalten sich also zu den alkalifreien wie der Alkaliglimmer zu dem alkalifreien Sprödglimmer nebst Margarit, und wie Alkalifeldspath zu Calciumfeldspath. Da in diesen hemimorphen Turmalinmolekiulen $3 \mathrm{Al}_{2}$ an dem einen, $B_{2}$ an dem anderen Ende gedacht werden muss, so kommt man hier zu derselben Ansicht über die Ursache der Hemimorphie des Turmalins, wie die schon von Schrauf ausgesprochene (diese Zeitschr. 9, 265).

Da das Bor-Atom nach der oben angeführten Elementartheorie (Abth. II) als tetartoeddrisch betrachtet werden kann, so kann es auch als hemimorphrhomboëdrisch gedacht werden, wenn man sich eine Tetraëderfläche als der basischen Flächenrichtung des Turmalinmoleküls entsprechend vorstellt. Die Polarelektricität des Turmalins kann dann als beruhend auf dem elektrischen Gegensatze des elektronegativen Bors und des elektropositiven Aluminiums angesehen werden. Dass der alkalifreie schwarze Turmalin im Allgemeinen sich weniger stark polarelektrisch zeigt als der Alkaliturmalin, kann von dem ungleichen Verhältnisse 2 wischen $A l_{2}$ und $B_{2}$ (resp. 2:1 und $3: 1$ ), oder auch davon herrühren, dass in dem $M g$-Turmalin ein Theil der Moleküle holomorph ist mit $A l_{2}$ an den beiden Enden. Uebrigens muss man annehmen, dass die $M g$ und $F e$ enthaltenden Molekularcomplexe mit denen des Alkaliturmalins isomorphe Verbindungen eingehen können, den molekularen Verbindungen zwischen dem Alkali- und Calciumfeldspath oder zwischen den verschiedenen Glimmerarten entsprechend, wodurch der geringe Alkaligehalt im $M g$-Turmalin oder der $M g$ - und $F e$-Gehalt in dem Alkaliturmalin erklärt werden. 
An den Turmalin schliessen sich in krystailographischer Hinsicht folgende von Brögger untersuchte und unter dem Namen Melanoceritgruppe zusammengefasste seltenen norwegischen Mineralien an (diese Zeitschr. 16, 462-489).

Cappelinit. Wenn man die von Brögger als $\frac{1}{3} P$ bezeichnete Pyramide als Grundform annimmt, wird $c=0,43$ oder nabe gleich der Hauptaxe des Turmalins $(=0,447)$, womit auch die prismatische Ausbildung des Cappelenitkrystalles in Uebereinstimmung steht. Dass der Cappelenit auch eine ähnliche Molekularfor'm wie die des Turmalins hat, zeigt sich, wenn

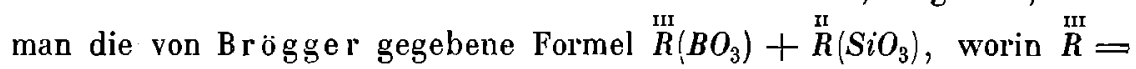
$Y(C e, L a, D i)$ und $\stackrel{M}{R}=B a\left(C a, N a_{2}, K a_{2}\right)$, in folgende Form bringt:

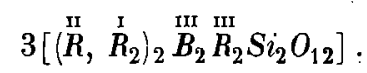

Diese Formel stimmt nämlich im Allgemeinen mit dem ersten Theile der Formel des Alkaliturmalins überein, und deutet wie diese auf rhomboëdrische Molekularcomplexe hin, zwillingsartig mit einander zu einem äusserlich scheinbar holoëdrisch-hexagonalen Krystall verbunden. Da die Cappelenitmoleküle nach obiger Structurformel an dem einen Ende $3 Y_{2}$, an dem anderen $3{ }^{\mathrm{mI}} \mathrm{B}_{2}$ enthalten, könnle man daraus auf eine Hemimorphie dieses Minerals schliessen, welche jedoch, wie Brögger bemerkt, nicht constatirt werden kann, da bisher ein einziger, nur an einem Ende ausgebildeter Krystall gefunden worden ist.

Der.Melanocerit ist rhomboëdrisch - tafelförmig (l. c.), welche vom Cappelenit abweichende Ausbildung durcb seine dreimal grössere Hauptaxe $(c=1,255)$ erklärt wird. Da er sich nun auch chemisch diesen Silicaten anschliesst, kann man für ihn eine ähnliche rhomboëdrische Molekularform annehmen, aber in Uebereinstimmung mit der des Glimmers zu einem Tripelmolekularcomplex in der Richtung der Hauptaxe vereinigt, wobei man als verbindende Glieder oder Kernmolekule zwischen den hemimorphen Partialmolekülen die von Brögger und $\mathrm{Gleve}$ in der Zusammensetzung des Melanocerits nachgewiesenen Fluorverbindungen annehmen kann (siehe unten über das ähnliche Verhältniss des Chondrodits).

Tri to mit steht in Hinsicht der Grösse der Hauptaxe, seiner hemimorphthomboëdrischen, nahe tetarloëdrischen Ausbildung, und wie es scheint auch in chemischer Hinsicht (l. c. 486) zwischen Cappelenit und Melanocerit. Seine Hemimorphie kann in Analogie mit der des Turmalins als auf der Anwesenheit verschiedener dreiatomiger Elemente, $\mathrm{Ce}_{2}, \mathrm{Y}_{2}$ etc. an dem einen, $\boldsymbol{B}_{2}$ an dem anderen Ende der Moleküle beruhend betrachtet werden. Auch Brögger sieht in dem Borgebalt (I. c. 489) eine Ursache der Hemimorphie des Tritomits, und erinnert hierbei an den nach Websky wahrscheinlich hemimorphen Jeremejewit $\left(\mathrm{Al}_{2} \mathrm{~B}_{2} \mathrm{O}_{6}\right)$, dessen Moleküle meiner Theorie nach ähnlich 
denen des Turmalins, Cappelenits und Tritomits sind, mit den oktaëdrischen resp. letraëdrischen Endatomen $\mathrm{Al}_{2}$ und $\boldsymbol{B}_{2}$ in rhomboëdrischer Stellung in der Richtung der Hauptaxe des hexagonalen Molekularcomplexes.

An die Turmalin- und Melanoceritgruppe schliessen sich die zirkonhaltigen Silicate Eudialyt und Ka tapleït, von welchen der Eudialyt der von R a mmelsberg gegebenen Formel gemäss als ein rhomboëdrischer, nahe oktaëdrischer Formencomplex betrachtet werden kann, aus sechs dureh $\mathrm{NaCl}$ als Kernmolekül verbundenen Parlialmolekulen bestehend; die bedeutende Grösse der Hauptaxe $(c=2,11)$ stimmt mit der gewöhnlich tafelförmigen Ausbildung und der basischen Spaltbarkeit überein. Der Katapleït $\left(\mathrm{Na}_{2} \mathrm{H}_{4} \mathrm{ZrSi}_{3} \mathrm{O}_{11}\right)$ mit $c=1,36$ nach Brög ger (l. c.) ist ebenfalls tafelrörmig, zeigt aber prismatische Spaltbarkeit, die ich jedoch eher als Absonderung betrachten möchte. Nach demselben Verf. zeigt Katapleït bei gewöhnlicher Temperatur monokline resp. trikline Anomalie, wird aber bei $140^{\circ}$ normal rhomboëdrisch, was erklärt werden kann durch die Annahme rhomboëdrischer Molekularcomplexe, denjenigen der vorigen Silicate äbnlich, und aus drei monosymmetrischen, in zwei hemiëdrisch-trikline Theile getheilten Partialmolekülen bestehend; diese Theile gehen bei höherer Temperatur in ein bomogenes Ganzes uber, indem die Elementaratome dabei eine mehr symmetrische Stellung einnehmen.

Eine den genannten hexagonalen Krystallen ähnliche Molekularstructur haben wir auch aus oben (Abth. I) angefuhrlen Gründen fur den Nephelin anzunehmen. Sowohl die Rammelsberg'sche Formel: $3 \mathrm{R}_{2} \mathrm{Al}_{2} \mathrm{Si}_{2} \mathrm{O}_{8}+$. $\mathrm{SiO}_{2}$, wie auch die von Rauff: ${ }_{4} \stackrel{\mathrm{I}}{\mathrm{R}_{2}} \mathrm{Al}_{2} \mathrm{Si}_{2} \mathrm{O}_{8}+\mathrm{SiO}_{2}$, wenn man jene mit 4, diese mil 3 multiplicirt, führen zur Annahme von zwölf mit dem halben Albitmolekul analogen hemimorphen Partialmolekulen, zu sechs quasimonoklinen $Z$ willingscomplexen vereinigt, und diese weiter zu einem holomorphen rhomboëdrischen Drillingscomplex (siehe Fig. 6) verbunden, um einen Complex von vier resp. drei $\mathrm{SiO}_{2}$-Molekülen als Kern- oder Centralmolekul angeordnet. Vielleicht ist es diesem Kernmolekül zuzuschreiben, dass die Nephelinkrystalle in krystallographischer Hinsicht mehr normal sind als Katapleït und andere Silicate, für welche man keine solchen Kernmolekule anzunehmen hat. Auch kann im Zusammenhange hiermit bemerkt werden, dass solche Silicate, welche Molekularcomplexe mit Kernmolekülen haben, wohl eine molekulare Zwillingsbildung, aber in der Regel keine solche im Grossen oder als ganze Krystalle zeigen, wodurch z. B. die auffallende Verschiedenheit in dieser Hinsicht zwischen dem Cordierit und Turmalin nebst ihren Verwandten und den Mineralien der Feldspathgruppe erklärt wird.

Molekularcomplexe mit Kernmolekülen hat man auch in den dem $\mathrm{Ne}-$ phelin sich anschliessenden Mineralien Cancrinit, Davyn und Mikro- 
sommit anzunehmen. Nach der von Rauff für Cancrinit aufgestellten Formel kann man in seinem Molekularcomplexe zwölf Partialmolkule ähnlich denen des Nephelins annehmen, und zwischen diesen sechs $\mathrm{CaCO}_{3}$ als Kernmoleküle, wobei bemerkt werden kann, dass der aus sechs Albitzwillingen bestehende Formencomplex (Fig. 6 ähnlich) mit dem Winkel $c: a=$ $63^{0} 17^{\prime}$ (dem Winkel $P: \infty P$ für Cancrinit und $\frac{1}{2} P: \infty P$ für Nephelin entsprechend) einen rhomboëderähnlichen Zwischenraum hat, mit Winkeln, welche sich denjenigen des Rhomboëders $-\frac{1}{2} R$ des Galcits nähern. In den Mikrosommitmolekulen sind gleichwie in denen des Sodaliths und seinen Verwandten (siehe oben) $\mathrm{NaCl}$ und $\mathrm{CaSO}_{4}$ als Kernmolekule anzunehmen.

Wir haben bisher eine Reihe rhombischer, rhomboëdrischer und regulärer Silicate betrachtet, welche mehr oder weniger deutlich eine aus monoresp. asymmetrischen Theilen bestehende innere Structur zeigen; wir kommen jetzt zu einer analogen Reihe von Silicaten einfacherer empirischer Zusanmensetzung, für welche wir eine ganz ähnlicbe Molekularstructur anzunehmen haben.

Der rhombische Hauptrepräsentant dieser Reihe ist der Olivin, welcher dem Cordierit analog ist, wie folgende Zusammenstellung zeigt :

Cordierit:

$$
\begin{aligned}
a: b: c & =0,5870: 1: 0,5585 \\
b^{1} & =1 \\
a b^{1} & =\sin 30^{0} 25^{\prime}=0,5063 \\
b c^{1} & =\sin 2911=0,4876
\end{aligned}
$$

olivin:

$$
\begin{aligned}
a: b: c & =0,5865: 1: 0,4657 \\
b^{1} & =1 \\
b c^{1} & =\sin 30^{0} 24^{\prime}=0,506 \\
a^{1} & =0,466 .
\end{aligned}
$$

Da nun für Cordierit (mit hemimorpher Molekularform) $b: b c: a b=$ $M g: A l_{2}: S i$ und $A l_{2}$ äquivalent mit $M g_{3}$ ist, kann man für Olivin $b: a: b c=$ $M g(F e): M g_{3}:$ Si setzen und kommt so fur diesen zu einer analogen rhombischen Molekularformel wie die für Cordierit; diese ist in der Zone $c-b$ der Cordieritform in der Zone $a-b$ sowohl krystallographisch wie chemisch ähnlich, wogegen die Zone $a-b$ des Olivins krystallochemisch der Zone $c-b$ des Cordierits nur annäherungsweise ähnlich ist. Auch unterscheidet jene sich von der Molekularformel des Cordierits dadurch, dass die vorige kein Kernmolekul hat. Wir kommen nun also zu folgender Constitutionsformel für Olivin:

$$
2 \stackrel{\text { II II }}{\mathrm{R}} \mathrm{Si}_{3} \mathrm{Si}_{2} \mathrm{O}_{8}=\stackrel{4}{4} \mathrm{II}_{2} \mathrm{SiO}_{4},
$$

und in Uebereinstimmung hiermit zur Annahme dreier Hauplarten: $\mathrm{MgMg}_{3} \mathrm{Si}_{2} \mathrm{O}_{8}$ (Forsterit), $\mathrm{FeMg} \mathrm{Si}_{2} \mathrm{O}_{8}$ (eigentlicher Olivin) und $\mathrm{FeFe}_{3} \mathrm{Si}_{2} \mathrm{O}_{8}$ (Fayalil), welche mil einander isomorphe Molekularverbindungen (gewöhnlicher Oliviv, Hyalosiderit, Hortonolit etc.) eingehen können. Hierzu kommt die Verbindung $\mathrm{MnMn}_{3} \mathrm{Si}_{2} \mathrm{O}_{8}$ in Knebelit, Trephroit und Stirlingit (Röpperit), in dem letzteren mit theilweiser Ersetzung des $M n$ durch $Z n$.

Die Berechtigung des Mittelgliedes $\left(\mathrm{FeMg}_{3} \mathrm{Si}_{2} \mathrm{O}_{8}\right)$, als eine selbstandige 
Abart aufgestellt zu werden, zeigt folgende Zusammenstellung der berechneten Procentzahlen mit zwei von Hjortdahl (I.) und Ziegenspeck (Il.) mitgetheilten Analysen (siehe Hin tze, Mineralogie S. 20).

\begin{tabular}{cccc}
\multicolumn{4}{c}{ Olivin : $\mathrm{FeMg}_{3} \mathrm{Si}_{2} \mathrm{O}_{8}}$. \\
$\mathrm{2SiO}_{2}$ & Berechnet: & I. & II. \\
$3 \mathrm{MgO}$ & 38,46 & 38,30 & 38,47 \\
$\mathrm{FeO}$ & $\frac{23,08}{100,00}$ & $\frac{38,29}{24,02}$ & 37,62 \\
\cline { 2 - 3 } & $\frac{24,83}{100,61}$ & $-100,92$
\end{tabular}

Bei Annahme dieser Zusammensetzung für Olivin (zum Theil) können die bekannten Olivinmetamorphosen in Serpentin und Amphibol auf folgende Weise erklärt werden.

$$
\begin{array}{cc}
\text { Olivin: } & \text { Serpentin: } \\
\mathrm{FeMg} \mathrm{Si}_{2} \mathrm{O}_{8}+2 \mathrm{H}_{2} \mathrm{O} & =\left(\mathrm{H}_{2} \mathrm{Mg}_{3} \mathrm{Si}_{2} \mathrm{O}_{8}+\mathrm{H}_{2} \mathrm{O}\right)+\mathrm{FeO} \\
\mathrm{FeMg} \mathrm{Si}_{2} \mathrm{O}_{8}+\mathrm{CaSi}_{2} \mathrm{O}_{5}=\mathrm{CaMg}_{3} \mathrm{Si}_{4} \mathrm{O}_{12}+\mathrm{FeO} .
\end{array}
$$

Bei diesen Metamorphosen ist es also, gleichwie bei der Metamorphose des Cordierits in Muscovit, derjenige Bestandtheil ( $\mathrm{Fe}$ ) des Atomcomplexes, welcher der längsten Normale $(b)$ entspricht und also eine geringere molekulare Attractionskraft als die ubrigen Atome hat, der fortgeht und durch $\mathrm{H}_{2}$ ersetzt wird. Hierdurch wird auch die schon oben angedeutete Aehnlichkeit zwischen der Molekularform des Serpentins resp. Kaolins' und der des Muscovits bestätigt. Weiter kommt man, da fur Amphibol $b_{2} c(021)$ : $\bar{b}_{2} c(0 \overline{2} 1)=59^{\circ} 34^{\prime}$ ist und also dem Winkel des Olivins $b c: \bar{b} c=60^{\circ} 48^{\prime}$ entspricht, für jenes Mineral zu folgendem Molekularverhältnisse: $b=C a$, $a=M g_{3}, b_{2} c=S i$, welches eine Molekularform andeutet ähnlich der für Talk (8), und die Metamorphose des Amphibols in diesen erklärt.

Durch die Annahme obengenannter Structurformel für Olivin kann man auch den sowohl krystallographischen wie chemischen Unterschied zwischen dem gewöhnlichen Olivin und dem Monticellit $\left(\mathrm{MgCaSiO}_{4}\right)$ ausdrücken, indem dieser dann als eine intermediäre Species zwischen Olivin und rhombischem Pyroxen betrachtet werden kann.

Dagegen schliesst sich die Chondroditgruppe am nächsten dem Olivin an, indem die drei von G. vom Rath angenommenen Typen Chondrodit, Humit und Klinohumit aus der Molekularform des Olivins herleitet werden können, wenn man sich diese als Doppelmolekul in der Richtung der $a$-Axe, durch das Tripelatom $M g_{3}$ zusammengehalten, und als Tripelmolekül in der Richtung der $c$-Axe vorstellt, und dann noch zu diesem resp. ein, zwei oder drei Doppelmoleküle des Olivins an jedem Ende dieser Richtung zufügt. Man kommt dann zu folgenden Axenverhältnissen der drei Typen : 


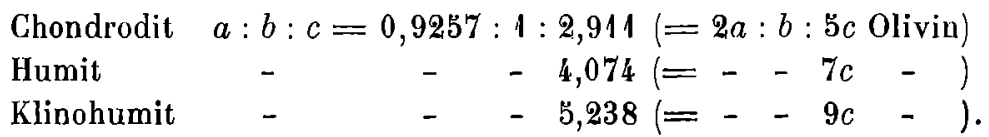

Nach dieser Auffassung haben wir also mehrere Grundformen für die Ghondroditarten anzunehmen, nämlich zwei Partialformen mit den Axenverhältnissen $2 a: b: c$ und $2 a: b: 3 c$ (im Vergleich zu dem des Olivins), deren Flächenrichtungen $b c$ und $b_{3} c$ die Zwillingsebenen der Chondrodittypen bilden, und die aus ihnen zusammengesetzten drei Molekularformen, welche die Krystallflächen der drei Typen bewirken. Diese Flächen stehen zu einander in einem anormalen Parameterverhältnisse $1: \frac{1}{3}: \frac{1}{5}: \frac{1}{7}$, welches durch die hexagonale Ausbildung der Partialformen in der Zone $b-c$ bedingt ist. Das Klinohumitmolekül ist gleich drei Partialmolekulen mit dem Axenverhältnisse $2 a: b: 3 c$, und verhält sich also zu diesen wie die Tripelmolekule der Glimmerarten zu ihren einzelnen aus der Feldspathform deducirten Partialformen.

Diese Auffassung steht mit der chemischen Zusammensetzung des Chondrodits in Uebereinstimmung, wenn man die von W ingard (Inaug.-Diss. 1884) für alle drei Typen angenommene Formel auf folgende Weise transformirt :

$$
(\mathrm{Mg}, \mathrm{Fe})_{19} \mathrm{Fl}_{4}(\mathrm{OH})_{2} \mathrm{Si}_{8} \mathrm{O}_{32}=2\left(2 \stackrel{\mathrm{II}}{\mathrm{R}} \mathrm{Mg}_{3} \mathrm{Si}_{2} \mathrm{O}_{8}\right)+\mathrm{Mg}_{3} \mathrm{Fl}_{4}(\mathrm{OH})_{2} \text {. }
$$

Diese Formel entspricht dann der einfachsten Partialform des Chondrodits, oder dem Doppelmolekttl des' Olivins mit Hinzufügung eines Magnesium-Fluorhydroxylmolekuls, welches als verbindendes Glied zwischen den Partialmolekülen in den zusammengesetzten Grundmolekülen der drei Chondrodittypen betrachtet werden kann.

Der Chondrodit und Klinohumit sind in optischer Hinsicht monoklin, der Humit sowohl optisch wie krystallographisch rhombisch, und man könnte mit Rücksicht hierauf den lelzteren als zusammengesetzt aus den zwei erstgenannten betrachlen, was, wenn man die zwei Theile obiger Constitutionsformel mit resp. $A$ und $B$ bezeichnet, auf folgende Weise ausgedruckt werden kann :

$$
\begin{aligned}
& \text { Chondrodit: Klinohumit: Humit: } \\
& 5(A+B)+9(A+B)=2 \cdot 7(A+B) .
\end{aligned}
$$

Hier möchte am besten der $\mathrm{Calam}$ in $\left(\mathrm{Zn}_{2} \mathrm{SiO}_{4}+\mathrm{H}_{2} \mathrm{O}\right)$ seinen Platz erhalten, obgleich er ziemlich isolirt steht und höchsens in einer Zone $c: b=0,4778: 1$ Aehnlichkeit mit der Zone $a: b=0,4657: 1$ des Olivins zeigt. Die in der $c$-Axe auftretende Hemimorphie des Calamins findet man bisweilen auch in der Richtung der $a$-Axe des Olivins (z, B. in einer Leucillava vom Vesuv). Während diese nur ausnahmsweise vorkommende Hemimorphie des Olivins wahrscheinlich auf verschiodenen Elementaratomen $\left(\boldsymbol{M} g_{3}\right.$ und $\left.\mathrm{Fe}_{3}\right)$ an den beiden Enden dieser Axe beruht, muss die Ursache der bei Calamin constanten Hemimorphie dem Elementaratom selbst zugeschrieben 
werden; und in der That kann das $Z n$-Atom, wie oben (Abth. II) angedeutet worden ist, als hemimorph beluachtet werden.

Die rhomboëdrischen Silicate von der allgemeinen Formel ${ }_{\mathrm{R}_{2}}^{\mathrm{S}} \mathrm{SiO}_{4}$ : Willemil und Troostit $(R=Z n$ und $M n)$, Phenakit $(R=B e)$, Dioptas $\left(R=H_{2}, C u\right)$ verhalten sich zu Olivin, wie Turmalin und dessen Verwandten zu Cordierit, d. b. ihre Molekularformen $\left(3 \stackrel{\mathrm{II}}{\mathrm{R}_{2}} \mathrm{SiO}_{4}\right)$ sind ähnlich der des Turmalins (11), aber mit $2 \mathrm{II}_{3}$ in den Endpunkten der $c$-Axe, welche der $a$-Axe des Olivins entspricht, wie auch die Verwandlung des rhomboëdri-schen Axenverhältnisses des Willemits $(a: c=1: 0,6698)$ in das rhombische $c: b: a=0,5774: 1: 0,4465$ zeigt. Die rhomboëdrischen Molekule können also als Complexe dreier halber mono- oder richtiger asymmetrischer Olivinmoleküle betrachtet werden, was die tetartoëdrische Ausbildung genannter Mineralien und ihre mehr oder weniger deutliche Anomalie erklärt, d. h. die Sonderung ihrer Krystalle in drei optisch zweiaxige Theile, was schon Breithau t bei Phenakit und Dioptas beobachtet hatte.

Diese Auffassung der Constitution genannter rhomboëdrischer Silicate wird durch den von Flink gefundenen Trime rit (diese Zeitschr. 18, 365) bestätigt, welcher, wie Flink und Brögger gezeigt haben, aus drei zu einem hexagonalen, pyramidal-bemiëdrischen Complex zusammengesetzten asymmetrischen Partialformen besteht. Da die von Brögger gefundene Zusammensetzung durch die Formel $\mathrm{Mn}_{3} \mathrm{Be}_{3} \mathrm{Si}_{3} \mathrm{O}_{12}$ ausgedrückt werden kann, erhält man auch für dieses Silicat eine mit der Molekularform des Turmalins analoge krystallochemische Structurformel.

Dem Dioptas schliesst sich der Pyrosmalith an, sowohl krystallographisch, indem $a: c=1: 0,5308 \mathrm{dem}$ Verhältnisse $a: c=1: 0,5342$ fül Dioplas gleich ist, wie auch chemisch, wenn man seine Formel wie folgt schreibt:

$$
3\left[\mathrm{H}_{2}(\mathrm{Fe}, \mathrm{Mn}) \mathrm{SiO}_{4}\right]+(\mathrm{Fe}, \mathrm{Mn})_{2} \mathrm{SiO}_{4}+(\mathrm{HO})_{2} \mathrm{Cl} .
$$

Die grôssere Complication der Zusammensetzung des Pyrosmaliths als die des Dioptas deulet eine grössere Mannigfaltigkeit in der molekularen Constitution an, nämlich einen Molekularcomplex zusammengesetzt aus Doppel- oder Tripelmolekülen in der Richtung der Hauptaxe, von $(\mathrm{HO})_{2} \mathrm{Cl}$ als Zwischenmolekule zusammengehalten, also eine Constitution analog mit der des Glimmermoleküls, welches auch die sehr vollkommene basische Spaltbarkeit des Pyrosmaliths anzeigt.

Der Eulytin $\left(\mathrm{Bi}_{4} \mathrm{Si}_{3} \mathrm{O}_{12}\right)$ verhält sich zu obigen rhomboëdrischen. Silicalen wie Sodalith zu Nophelin oder Granat (Helvin) zu Biotit resp. Turmalin; indem er nach den Untersuchungen von Bertrand in seinem optisch anomalen Zustande als aus vier rhomboëdrischen Krysallen zusammengesetzt betrachtet werden kann, und die Formen $R,-\frac{1}{2} R$ und $-2 R$ des Dioptas sich dem Rhombendodekaëder, Trigondodekaëder und Hexaëder nähern. 
Wie Berlrand und nach ihm Brauns gezeigt haben, theilen sich die rhomhoëdrischen Parlialindividuen weiter in drei monosymmetrische Theile, den Trigondodekaëderflächen des Eulytinkrystalles entsprechend, diese verhalten sich wahrscheinlich zu dem monoklinen, mit Eulytin isomeren $\Lambda \mathrm{gricoli}$. wie die monoklinen Partialindividuen des Granats zu dem Partschin. Mit Rucksicht hierauf könnte man das Eulytinmolekül $=12 \mathrm{Bi}_{4} \mathrm{Si}_{3} \mathrm{O}_{12}$ setzen, und den Eulytinkrystall als aus einem solchen Centralmolekül bestehend betrachten, an welches sich einzelne Partialmolekule symmetrisch anschliessen.

An Eulytin und dadurch auch an die rhomboëdrischen resp. pseudohexagonalen Silicate von der allgemeinen Formel $\stackrel{\mathrm{II}}{R}_{2} \mathrm{SiO}_{4}$ schliesst sich der $\mathrm{Helvin}$, dessen Formel $=3\left[(\mathrm{Mn}, \mathrm{Fe}, \mathrm{Be})_{2} \mathrm{SiO}_{4}\right]+(\mathrm{Mn}, \mathrm{Fe}) \mathrm{S}$, aber auch $=(M n, F e)_{3}{ }_{3} \mathrm{Be}_{3} \mathrm{Si} i_{3} \mathrm{O}_{12}+(M n, F e) S_{\mathrm{II}}$ geschrieben werden kann, in welchem Falle das Silicat als Granat mit $\mathrm{Be}_{3}$ für $A l_{2}$ betrachtet werden kann. Dass man in der That berechtigt ist, Beryllium von Mangan und Eisen abzusondern, zeigt sich daraus, dass den Analysen nach der $B e O$-Gehalt ziemlich constant ist $(11-14 \%$, wäbrend $\mathrm{FeO}$ von $4-15 \%$ und $\mathrm{MnO}$ von 37 bis $27 \%$, also in umgekebrtem Verhältnisse, wechseln. Man hat also einen Mangan- und einen Eisen-Helvin zu unterscheiden, welche mit einander molekulare Verbindungen eingehen, und wozu noch der $Z n$-Helvin in dem Danalith kommt.

Folgende Zusammenstellung zeigt die Uebereinstimmung der aus dieser Formel berechneten Procentzahlen des $M n$-Helvins mit einer von $\mathrm{R}$ a mmels. berg ausgeführten Analyse eines eisenarmen Helvins aus Süd-Norwegen.

\begin{tabular}{|c|c|c|}
\hline $3 \mathrm{SiO}_{2}$ & $\begin{array}{l}\text { Mn-Helvin. } \\
\text { Berechnet: } \\
\quad 32,43\end{array}$ & $\begin{array}{c}\text { Helvin } \\
\text { nach Ram me l s berg: } \\
33,13\end{array}$ \\
\hline $3 B e O$ & 13,51 & 11,46 \\
\hline $3 \mathrm{MnO}$ & 38,38 & $35,50(\mathrm{FeO}=4,00)$ \\
\hline$M n S$ & 15,68 & 15,58 \\
\hline & 100,00 & \\
\hline
\end{tabular}

Die Elementaratome des Silicats des Helvins lassen sich dieser Formel gemäss in Uebereinstimmung mit den Molekularformen des Turmalins und Dioptas anordnen, wodurch man zu einer hemimorphen rhomboëdrischen Molekurlarform kommt, mit $B e_{3}$ an dem einen, $M n S$ an dem anderen Ende und $3 \mathrm{Mn}$ nebst $3 \mathrm{Si}$ an den Seiten. Vier solche Moleküle bilden, wie bei Eulytin, einen tetraëdrischen Molekularcomplex mit 4MnS als Kernmolekul.

Hier möchte ich noch zwei Silicate anfuhren, Beryll und Ax in it, von welchen jener mit Cordierit, besonders dem pseudohexagonalen, vulkanischen, dieser mit Turmalin einige Beziehungen hat. Diese nebst den naturlichen Aetzfiguren und die optische Anomalie des Berylls führen zur Annahme folgender Molekularform in Quer- und Längsschnitt. 
Fig. 12.

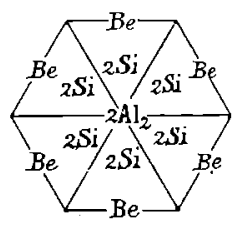

[ig. 13.

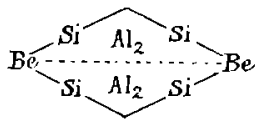

Beryll : $2\left(B e_{3} A l_{2} \mathrm{Si}_{8} \mathrm{O}_{18}\right)$.

Dieser Molekularcomplex ist holoëdrisch-hexagonal, aber aus drei quasi-rhombischen Parlialmolekuilen zusammengesetzt, welche mit den oben fur Muscovit und Kaolin angenommenen Molekularformen (4 und 9) analog sind, und dadurch die Metamorphose des Berylls in diese Mineralien erklären, wobei man annehmen kann, dass die $A l$-Atome aus einer hexagonalen Flächen- in eine rhombische Kantenstellung übergehen. Wohl kennt man kein rhombisches Silicat von der Zusammensetzung $B e A l_{2} \mathrm{Si}_{2} \mathrm{O}_{8}$, aber ein damit analoges, nämlich Bertrandit, denn dieser kann durch die Formel $\mathrm{BeBe}_{3} \mathrm{Si}_{2} \mathrm{O}_{8}+\mathrm{H}_{2} \mathrm{O}$ und durch eine ähnliche bemimorphe Molekularformel wie die für Margarit (b) ausgedrückt werden, und verhält sich zu Beryll wie der hemimorphe rhombische Cordierit zu dem quasi-hexagonalen, in vulkanischen Gesteinen vorkommenden Cordierit. Auch ist er krystallographisch der genannten hypothetischen rhombischen Form des Berylls ähnlich, indem sein Prismenwinkel (=59021') sich dem Pyramidenwinkel $\left(59^{\circ} 54^{\prime}\right)$, und sein Domenwinkel $\left(=61^{\circ} 21^{\prime}\right)$ dem Prismenwinkel $\left(60^{\circ}\right)$ des Berylls nähern; ein Drillingscomplex von Bertrandit-Individuen mit dem Doma $\{011\}$ als Zwillingsebene ist also der Grundform des Berylls analog.

Ich muss hier noch, die optische Anomalie des Berylls*) betreffend, bemerken, dass, da ich diese in Uebereinstimmung mit meiner Ansicht uber die Molekularconstitution dieses Minerals als beruhend auf einem Zerfallen in rhombische Partialmolekule betrachte, ich doch nicht annehme, dass diese Partialmoleküle dern oben genannten hypothetischen Silicat vollkommen entsprechen, dessen Zusammensetzung $\left(=2 \mathrm{BeS}_{2} \mathrm{O}_{5}+\mathrm{Al}_{2} \mathrm{O}_{3}\right)$ von der des Berylls $=\mathbf{2}\left[3\left(\mathrm{BeSi}_{2} \mathrm{O}_{5}\right)+\mathrm{Al}_{2} \mathrm{O}_{3}\right]$ verschieden sein muss, sondern dass der anomale Beryll, wie im Allgemeinen die anomalen Krystalle, einen inlermediären Zustand bildet zwischen dem normalen hexagonalen und dem rhombischen, was daraus hervorgeht, dass er an verschiedenen Stellen ein verschiedenes optisches Verhalten zeigt, und im Inneren des Krystalles gewöhnlich weniger anomal als in den äusseren Theilen ist.

Der Axinit nähert sich durch seine Winkel $P: u=44^{0} 29^{\prime}$ und $P: r=$

*) Dass diese Anomalie nicht, wie B r a un s (Die optischen Anomalien) es ansieht, auf einer Spannung oder Druck beruht, scheint mir daraus hervorzugehen, dass, wie Bü cking beobachtet hat, Druck keine bleibende Veränderung in der Molekularstructur des Berylls zu verursachen vermag. 
$45^{0} 15^{\prime}$ dem Grundrhomboëderwinkel des Turmalins, eine Analogie, welche man auch in der chemischen Zusammensetzung wiederfindet, wenn man die von Rammelsberg gegebene empirische Formel auf folgende Weise schreibt: $3 R_{2} R_{2} S i_{2} O_{8}+R_{3} S_{2} i_{2} O_{7}$. Wenn man sich die Elementaratome des ersten Theiles dieser Formel in Uebereinstimmung mit denen in dem Grundmolekül des Turmalins um jene des zweiten Theiles als Kernmolekul angeor'dnet denkt, so könnte die Asymmetrie des Axinits als beruhend auf verschiedenen $\stackrel{\mathrm{Ir}}{R}$ - und $\mathrm{III}_{2}$-Atomen $\left(2 \mathrm{Ca}+\mathrm{Fe}\right.$ und $\left.2 \mathrm{Al}_{2}+\mathrm{B}_{2}\right)$ angesehen werden, während sie in dem Turmalinmolekül gleich sind, oder durch molekulare $\mathrm{Z}$ willingsbildung zu einem asymmetrischen Ganzen vereinigt sind. Bei dem Axinit scheint weder eine molekulare Zwillingsbildung noch eine solche im Grossen vorzukommen; in dieser letzteren Hinsicht zeigt sich eine auffallende Verschiedenheit von den einfacher zusammengesetzten triklinen Silicaten Anorthit und Cyanit.

Uebrigens steht der Axinit allzu isolirt oder ohne directe Beziehungen zu anderen Silicaten, um eine nähere Erörterung seiner Molekularstructur zuzulassen.

Wir haben die Silicate mit Winkeln in einer Hauptzone (Prismenzone) gleich oder nahe $120^{\circ}$ betrachtet, und kommen jetzt zu den mit $90^{\circ}$ oder nahe $90^{\circ}$ in dieser Zone. Wenn wir auch hier mit den der Feldspathgruppe am nächsten stehenden Alkalisilicaten anfangen, so haben wir zuerst Spodumen und Petalit zu betrachten, welche in krystallochemischer Hinsicht der Feldspathgruppe so nahe stehen, dass sie eher Lithion-Feldspath als Alkalipyroxen genannt werden können.

Aus den oben (Abth. I) angefuhrlen Relationen zwischen den Winkeln der Hauptflächen des Orthoklases und Spodumens findet man, dass die Zonen $a-b, b-c, a-c$ des Orthoklases den Zonen $c-b, b-a, c-a$ des Spodumens entsprechen, und weiter, dass das Verhällniss zwischen den Normalen des Orthoklases $c^{2}: b: a b$ dem Normalenverhältniss des Spodumens $a: b: b c$ ähnlich ist, indem für Spodumen $b^{1}=1, a^{1}=1,124 \times$ $\sin 69^{\circ} 48^{\prime}=1,059, b c^{1}=\sin 30^{\circ} 55^{\prime}=0,5138$ sind; und wir kommen dadurch von der oben bei Orthoklas angenommenen Stellung der Elementaratome zu folgendem Molekularverhältnisse für Spodumen :

$$
2 b=2 L i, \quad a=A l_{2}, \quad 4 b c=4 S i,
$$

welches der empirischen Formel $\mathrm{Li}_{2} \mathrm{Al}_{2} \mathrm{Si}_{4} \mathrm{O}_{12}$ entspricht.

Bemerkt werden kann noch, dass der Winkel $a: c=69^{\circ} 40^{\circ}$ bei Spodumen dem $\mathrm{Oktaëderwinkel} 70^{\circ} 30^{\prime}$ des hypothetischen Aluminiumatoms näher steht, als der entsprechende Winkel $c: a(001: 100)$ bei Orthoklas, welches eine etwas veránderle Stellung dieser Atome in den entsprechenden Zonen anzeigt, von der Abwesenheit der $\mathrm{Si}_{2}$-Atome des Orthoklases in dem 
Grundmolekul des Spodumens bedingt. Hierauf beruht wohl auch der Unterschied der entsprechenden Winkel $a b: a \vec{b}=93^{\circ} 12^{\prime}$ bei Spodumen und $b_{2} c: \bar{b}_{2} c=90^{\circ} 3^{\prime}$ bei Orthoklas.

Die nahe Verwandtschaft des Spodumens mit Alkalifeldspath zeigt sich auch aus den von Brush und Dana (diese Zeitschr. 5) beschriebenen Metamorphosen des Spodumens in Albit resp. Mikroklin, Muscovit unk Eukryptit, welcher letztere in krystallochemischer Hinsicht zu Spodumen in demselben Verhältnisse wie Nephelin zu Albit zu stehen scheint.

Mit den oben (Abth. I) angeführten Verbälınissen zwischen Krystallform und Molekularvolumen (1:2) des Spodumens und Petalits stebt ihre chemische Zusammensetzung in Uebereinstimmung, denn der Petalit $\left(\mathrm{Li}_{2} \mathrm{Al}_{2} \mathrm{Si}_{8} \mathrm{O}_{20}\right)$ kann als Spodumen $+4 \mathrm{SiO}_{2}$ betrachtet werden und verhält sich also zu diesem wie Albit zu Anorthit, was zu der Annahme führt, dass das Petalitmolekul gleichwie das des Alkalifeldspaths aus zwei Theilen in der Richtung der $c$-Axe, der $a$-Axe des letzteren entsprechend, besteht.

Nach den schon oben (Abth. I) dargestellten Axenverbältnissen des Wollastonits und Spodumens stehen sie in krystallochemischer Beziehung in demselben Verhältnisse wie Calciumfeldspath zu Alkalifeldspath und Calciumglimmer zu Alkaliglimmer. Das Verhältniss zwischen Wollaston it $\left(\mathrm{CaSiO}_{3}\right)$ und $\mathrm{Rhodonit}\left(\mathrm{MnSiO}_{3}\right)$ ist auch schon oben angefuhrt. Es zeigt sich durch folgende Zusammenstellung ihrer Axenverhăltnisse (nach $\mathrm{Ham}$ berg l. c.):

$\begin{array}{llc}\text { Wollastonit } & 1,114: 1: 0,483 ; & \beta=69^{\circ} 48^{\prime}, \\ \text { Rhodonit } & 1,147: 1: 0,456 ; \quad \alpha=85^{0} 46^{\prime}, \beta=68^{\circ} 24^{\prime}, \gamma=86^{\circ} 4^{\prime} \text {. }\end{array}$

Der Wollastonit und der Rhodonit stehen also zu einander in demselben Verhältnisse wie Orthoklas zu Albit oder der im Hyalophan vorhandene Baryumorthoklas zu Anorthit, d. h. sie sind als isodimorphe Silicate zu betrachten, was auch durch das Vorkommen des $\mathrm{Ca}$ in Rhodonit und Bustamit (2 $\mathrm{MnSiO}_{3}+\mathrm{CaSiO}_{3}$ ) angezeigt wird. Da nun, wie oben angeführt worden ist, der Wollastonit als bemimorph-monoklin in der Richtung $\operatorname{der} b$-Axe zu betrachten ist, so ist auch in Uebereinslimmung hiermit der Rhodonit als hemiëdrisch-triklin in Analogie mit dem Anorthit anzusehen. Dieser Ansicht gemäss sind die beiden Spaltungsflächen des Rhodonits ( $c$ und b) nicht als Prismen- sondern als Pinakoidflächen zu betrachten, indem sie den Flächen $b(010)$ und $a(100)$ des Wollastonits und also auch den Spaltungsflächen $b(010)$ und $c(001)$ des Anorthits krystallochemisch analog sind; und folglich entsprechen das $C a$ - und $M n$-Atom der $b$-Normale resp. $b$-Fläche des Wollastonits und des Rhodonits.

Wie Wollastonit und Rhodonit sich zu einander wie der monokline zu dem triklinen Feldspath verhalten, so verhalten sich Rhodonit und Babingtonit $\left(\mathrm{mRSiO}_{3}+\mathrm{Fe}_{2} \mathrm{Si}_{3} \mathrm{O}_{9}\right)$ wie Anorthit zu Albit. Die Formel des 
Babingtonits kann nämlich auch zum Theil $=\stackrel{\text { If }}{R} \stackrel{\text { II }}{R}{ }_{2} S i_{4} O_{12}$ gesetzt werden, und verhält sich dann zu Spodumen wie Rhodonit zu Wollastonit. Die Veränderlichkeit seiner Zusammensetzung kann dann durch molekulare Verbindungen zwischen dieser Abart des Bubingtonits und dem Rhodonit, den Plagioklasverbindungen zwischen Albit und Anorthit entsprechend, erklärt werden. In krystallographischer Beziehung unterscheidet sich der Babingtonit von dem Rbodonit nicht mehr als Albit von Anorthit. Da nun auch monokline Molekularformen von der Zusammensetzung $\mathrm{RFe}_{2} \mathrm{Si}_{4} \mathrm{O}_{12}$, nämlich die des Akmits und Aegirins, vorkommen, so kann man den krystallochemischen Unterschied zwischen diesen und dem triklinen Pyroxen (Babingtonit) so bezeichnen, dass die monoklinen Formen aus zwei gleichen hemimorphen, die triklinen aus zwei ungleichen hemiëdrischen, zu einem holomorphen resp. holoëdrischen Molekularcomplex in der Richtung der $b$ - resp. $a$-Axe vereinigten Partialmolekülen bestehen.

Dass nun auch die Molekularform des eigentlichen monoklinen $\mathrm{Ca}(\mathrm{Mg}, \mathrm{Fe})-$ Pyroxen (Augit, Diopsid, Diallag etc.) aus zwei hemimorphen zu einem holomorphen Grundmolekul in der Richtung der Symmetrieaxe $(b)$ vereinigten Partialmolekulen besteht, geht aus der schon oben nachgewiesenen Aehnlichkeit zwischen den.Winkeln der Grundform des Pyroxens und denjenigen eines Bavenoer. Zwillings des Anorthits (von dem Anorthoit-Typus Fig. 2) hervor.

Dagegen ist der Winkel der Pyramidenflächen des Pyroxens von dem Winkel der entsprechenden Prismenflächen des Anorthitzwillings verschieden, was eine verschiedene Stellung der $S i$-Atome des Pyroxenmoleküls von der im Feldspathmolektil andeutet. Dieses geht auch aus dem oben (Abth. II) angefubrten verschiedenen Verhältnissen der Normalen $a b^{1}: b^{1}=$ 1: 2 bei Feldspalh und den der entsprechenden $\bar{a} b c^{1}: a b^{1}=2: 3$ bei Pyroxen hervor. Aber jenes Verhältniss nähert sich dem Normalenverhälnisse $\bar{a} b c^{1}: b^{1}(=0,49: 1)$ für Pyroxen, was zu der Annahme $a b c=S i$, $b=C a$ führt, und also zu derselben Stellung für die $C a$ - (und $M n$-) Atome wie im Wollastonit und Rhodonit, wogegen die $M g$ - resp. $F e$-Atome eine prismatische oder $a b$-Stellung einnehmen mussen, da jener Pyroxen im Gegensatze zu der pinakoidalen Ausbildung des Wollastonits und Alkalipyroxens eine prismatische Ausbildung hat; das Verhältniss der reciproken Werthe der Normalen $a b_{1}: \bar{a} b c_{1}=2: 3$ führt weiter zur Annahme einer Kantenstellung für $M g$, einer Flächenstellung für $S i$, welche also mit resp. zwei und drei Atomkräften (Dynamiden) angreifen.

In der Richtung der Symmetrieebene oder $a$-Axe ist, wie schon oben bei Kaolin angedeutet worden, die Molekularform des Pyroxens in seiner einfachsten Form gleichwie die des monoklinen Kaolins (9) als hemiëdrisch oder nur zur Hălfte ausgebildet zu betrachten, indem wir fưr die mono- 
klinen und triklinen pyroxenarligen Silicate in der Richtung der a-Axe, entsprechend den Feldspatharlen in der Richtung der $c$-Axe, ein einfaches hemimorphes Molekül und ein holomorphes Doppelmolekül anzunehmen haben. Jene Molekularform fur die monoklinen Pyroxene $\left(2 \mathrm{CaRSi}_{2} \mathrm{O}_{6}\right)$ ist unten mit der futr rhombischen Pyroxen $\left(4 \mathrm{RSiO}_{3}\right)$ zusammengestellt, welche einer molekularen Zwillingsform des monoklinen Pyroxens oder einem Doppelzwilling resp. Bavenoer Vierling des Anorthits entspricht.

Fig. 14.

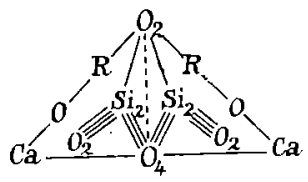

Monoklinẹr Pyroxen.
Fig. 15.

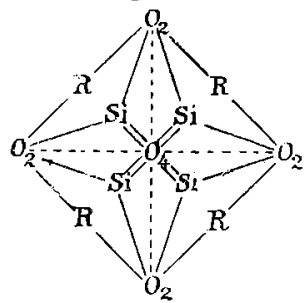

Rhombischer Pyroxen.

Folgende Zusammenstellung der Winkel der Grundform des monoklinen Pyroxens mit den entsprechenden des rhombischen Pyroxens kann die Richtigkeit dieser Auffassung bestätigen und zugleich anzeigen, dass die Si-Atome bei rhombischem Pyroxen den Flächen der oft vorherrschenden Pyramide $\mathbf{p 2}=(122)(e)$ entsprechen, wobei das Axenverhältniss $a: b: c=1,0302$ : $1: 0,5877$ gesetzt ist.

Monokliner Pyroxen :

$$
(110):(1 \bar{T} 0)=92054^{\prime}
$$$$
(T 11):(T T 1)=59 \quad 12
$$

Bhombischer Pyroxen :

$(110):(1 T 0)=91^{\circ} 0^{\prime}$

$(122):(1 \overline{2} 2)=592$

Wean man in der Molekularform (14) $C a$ als der $b$-Normale entsprechend ansieht, kommt man zu der Molekularform des schon oben angeführten Monticellits $\left(4 \mathrm{CaMgSiO}_{4}\right.$ ), welche aus zwei in der $a$-Richtung hemimorphen Partialmolekülen besteht. Im Gegensatze hierzu und in Uebereinstimmung mit den Molekularformen des Alkalipyroxens sind in den $\mathrm{Fe}_{2}$ - und $\mathrm{Al}_{2}$-haltigen rhombischen Pyroxenen, wie auch in den monoklinen (Diallag), die $\mathrm{Fe}_{2}$ - und $\mathrm{Al}_{2}$-Atome in die Endpunkte der $a$-Nolmale zu versetzen, wodurch die prismatische Ausbildung in eine pinakoidale ubbergeht. Hierbei können Doppel- resp. Tripelcomplexe in dieser Richtung entstehen, was die lamellare Absonderung und die bisweilen glimmerartige Spaltbarkeit parallel der $a$-Fläche bei diesen Abarten erklärt; dieses ist besonders bei dem sogenannten Bastit der Fall, welcher wohl auch, nach den optischen Verhältnissen und übrigen physikalischen Eigenschaften zu folgern, in wirklichen Glimmer (Phlogopit) ubergehen kann.

Von den Molekularformen des monoklinen und rhombischen Pyroxens kommen wir, wie schon oben angedeulet ist, zu den entsprechenden 
Amphibolformen durch Verdoppelung der vorigen in der Richtung der $b$ Axe, wobei angenommen werden muss, dass an dem Uebergange des Pyroxens in Amphibol (Uralit) drei Pyroxenmolekule Theil nehmen, wie folgende Gleichung zeigt :

$$
3 \mathrm{Ca}_{2} \mathrm{Mg}_{2} \mathrm{Si}_{4} \mathrm{O}_{12}=2 \mathrm{CaMg}_{3} \mathrm{Si}_{4} \mathrm{O}_{12}+4 \mathrm{CaSiO}_{5} .
$$

$2 \mathrm{Mg}_{3}$-Atome verbinden sich hierbei, wie schon oben bei Olivin angedeutet ist, in der $a$-Richtung, während die $C a$ - und $S i$-Atome den $b$ - und $b_{2} c$-Normalen resp. Flächen entsprechen, welche letzteren Flächen den Pyramidenflächen des Pyroxens bei den zwillingsartigen Zusammenwachsungen des Amphibols und Pyroxens parallel gehen.

Ein Vergleich dieser Molekularform des Amphibols mit der des Biotits (6 und 7) bestätigt dieselbe. Wie nämlich diese Grundformen des Biotits aus drei feldspathartigen Partialformen (110).(001) besteben, so kann man sie auch als aus Partialformen (021).(100) bestehend betrachten, wenn man diese so stellt, dass ihre Domenflächen (021) den Prismenflächen der Biotitform parallel gehen und ihre Symmetrieebenen in der Mitte zwischen zwei Feldspathformen stehen. In diesen intermediären Aichtungen zeigen die dem Biotitcomplex entsprechenden Drillingscomplexe des Plagioklases einen Winkel (ungefähr 730), welcher sich dem entsprechenden Winkel $a: c=$ $75^{\circ} 10^{\prime}$ des Amphibols nähert. Dieses Verbältniss zwischen den Amphibolund Biotitmolekulen erklärt die Metamorphosen des Amphibols in Biotit.

An Pyroxen, am nächsten an Alkalipyroxen (Akmit und Aegirin), schliesst sich der Låvenit (Brögger), mit deutlicher, orthopinakoidaler Spaltbarkeit und wahrscheinlich auch wie Spodumen und Alkalipyroxen pinakoidaler Stellung der positiven Radicale. An diesen schliesst sich weiter der Wöhlerit mit mimetisch-regulärer Form $\left[(110):(110)=90^{0} ; \beta=71^{\circ}\right]$, was eine tetragonale Stellung der positiven Atome ( $\mathrm{Ca}$ und $\mathrm{Na}$ ) in der Prismenzone, eine rhombische der $S i$ - und $Z r$ - resp. $N b$-Atome in der Zone $a-c$.andeutet.

An die rhombischen Amphibole (Anthophyllit resp. Gedrit) schliesst sich wahrscheinlich der Titan- und Zirkon-haltige Astrophyllit, indem dieses Mineral sich zu Anthophyllit in ähnlicher Weise wie Bastit zu Bronzit zu verhalten scheint.

Eine Reibe, analog mit der von Rhodonit, Pyroxen und Amphibol, bilden die hauptsächlich aus Aluminiumsilicaten bestehenden Mineralien Cy anit, Andalusit (Sillimanit) und Staurolith. Wenn man das Andalusitmolekul mit einem Bavenoer Vierlingscomplex des Anorthits vergleicht, in welchem die den $A l$-Atomen entsprechenden $c$-Flächen nach aussen liegen, kann das Grundmolekül des Gyanits mit einem von den vier einzelnen Theilen dieses Complexes, und die deutlichste Spaltungsfläche $(M)$ desselben mit der $c$-Fläche $(\boldsymbol{P})$ des Anorthits verglichen werden. Dieser Vergleichung 
gemäss entsprechen die vier prismatischen Spaltungsrichtungen in der Grundform des Andalusits den vier $\mathrm{Al}$-Atomen in der Formel $2 \mathrm{Al}_{2} \mathrm{SiO}_{5}$. Diese Grundform $(a: b: c=0,9861: 1: 0,7024)$ nähert sich einem Hexaëder in diagonaler Stellung, die Hexaëderfächen den Prismenflächen des Andalusits entsprechend, und mit den Elementaratomen wie die Molekular-

Fig. 16.

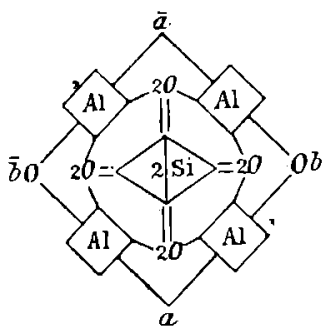

Andalusit: $2 \mathrm{Al}_{2} \mathrm{SiO}_{5}$ formel 16 anzeigt angeordnet, nämlich den vier Aluminiumatomen in tetragonaler und den zwei Siliciumatomen in rhombischer Stellung, jene an den Endpunkten der Seitennormalen, diese an denjenigen der basischen Flächennormalen, also in gleichem Abstande vom Centrum.

Dass ausser dieser Molekularform noch andere fur Andalusit resp. Ghiastolith vorkommen, zeigt eine Analyse eines Andalusits von Gurban Schivar von J e r e mejew (Russ. min. Ges. 1863, 140) mit $\mathrm{SiO}_{2} 53,60, \mathrm{Al}_{2} \mathrm{O}_{3} 43,10, \mathrm{Fe}_{2} \mathrm{O}_{3}$ 4,01, welche zu der Formel $\mathrm{Al}_{2} \mathrm{Si}_{2} \mathrm{O}_{7}$ führt mit $\mathrm{SiO}_{2} 54,05$ und $\mathrm{Al}_{2} \mathrm{O}_{3} 45,95 \%$. Diese Abart unterscheidet sich von den gewöhnlichen Andalusiten durch einen Prismenwinkel, welcher sich mehr dem fur Pyroxen năhert, und also wobl auch durch eine der rhombischen Pyroxenform (15) ähnliche Molekularform mit $A l=a b, S i=a b c$. Hierzu kommen der von mir analysirte Sillimanit von St. Michel und der von Ko monen analysirte Xenolith in einem erratischen, wahrscheinlich von St. Michel herstammenden Block bei Peterhof, und man erhält so folgende homologe Serie für Andalusit-Sillimanit :

$$
\begin{aligned}
& \left(\mathrm{Al}_{2}\right)_{2} \mathrm{Si}_{2} \mathrm{O}_{10} \text { (Andalusit und Sillimanit z. Th.), } \\
& \left(\mathrm{Al}_{2}\right)_{2} \mathrm{Si}_{3} \mathrm{O}_{12} \text { (Xenolitb, Fibrolith z. Th.), } \\
& \left(\mathrm{Al}_{2}\right)_{2} \mathrm{Si}_{4} \mathrm{O}_{14} \text { (Andalusit und Chiastolith z. Th.). }
\end{aligned}
$$

In dieser Reihe bildet der Xenolith das Zwischenglied, dem Andesin der Plagioklasserie entsprechend, und kann wie dieser als aus den zwei isomorphen Grenzgliedern zusammengesetzt angenommen und sein Grundmolekül daher gleich $2\left[\left(\mathrm{Al}_{2}\right\rangle_{2} \mathrm{Si}_{3} \mathrm{O}_{12}\right]$ gesetzt werden. Wenn man sich diese zwei Atome durch $\mathrm{Al}_{2}$ in der Richtung der Normale der Spaltungsfläche verbunden denkt, könnte dieses die deutliche pinakoidale Spaltbarkeit des Sillimanits erklären.

Dass noch complicirtere Aluminiumsilicate in der Natur vorkommen, zeigt der D u mortierit $\left(\mathrm{Al}_{8} \mathrm{Si}_{3} \mathrm{O}_{18}\right)$, welchen man als Mittelglied einer der vorigen analogen homologen Reihe betrachten $k$ ann, mit $\left(A l_{2}\right)_{4}$ an Stelle von $\left(A l_{2}\right)_{2}$. Diese homologen Reihen entsprechen denen der Aluminiumbydrosilicate Kaolin, Halloysit, Kollyrit etc. Auch die von Rammelsberg aus zwei Analysen deducirte Formel $\mathrm{Al}_{16} \mathrm{Si}_{9} \mathrm{O}_{42}$ zeigt eine complicirte Molekularform an aus acht Partialmolekullen, einem Doppelzwillingscomplex von Plagiosklas entsprechend, um ein Molekul $\mathrm{SiO}_{2}$ als Kernmolekul. 
Das Staurolithmolekül kann man als einen Complex von Andalusitmolekülen, durch das Doppelatom $A l_{2}$ vereinigt, ansehen, gleichwie die Olivinmolekule durch $\mathbf{M} g_{3}$ in den Molekularcomplexen der Gbondrodittypen. Auch für den Staurolith kommt man nämlich zur Annahme zweier Grundformen, einer so zu sagen inneren, auf welcher die Zwillingsebenen, und einer äusseren, worauf die Krystallfächen beruhen. Der Staurolith zeigt übrigens bisweilen eine Structur analog mit der des Chiastolitbs, welche zu den Krystallfächen in einer ähnlichen Beziehung, wie die Structur des anomalen Granats, Vesuvians etc., steht.

Diese Complication in der molekularen Constitution des Stauroliths entspricht einer ähnlichen in der chemischen Zusammensetzung, wie die Analysen von Rammelsberg, Coloriano und Friedel anzeigen. Ausser den dreiatomigen Elementen $\mathrm{Al}_{2}$ und $\mathrm{Fe}_{2}$ kommen noch die zweiatomigen $M g$ und $F e$ vor, welche ubrigens nach Grünhut (diese Zeitschr. 9) auch im Andalusit vorkommen. Es könnte jedoch in Frage gestellt werden, ob diese wirklich in den Molekulen des Andalusits resp. Stauroliths vorkommen, oder eher in mit diesen vereinigten homöomorphen rhombischen $\mathrm{Py}_{-}$ roxenmolekülen.

Da das Andalusitmolekưl mimetisch-regulär ist, könnte man a priori vermuthen, dass durch molekulare Zwillingsverwachsung ein regulärer Molekularcomplex entstehen könnte; und in der That scheint der tetraëdrische $\mathrm{Zunyit}$ aus einem solchen zu bestehen, um eine Hydroxyl-Fluorverbindung als Kernmolekuil vereinigt.

Als einen aus Andalusitmolekülen zusammengesetzten Molekularcomplex kann man auch den des Topas ansehen. Nach der von Rammelsberg angenommenen Formel $5 \mathrm{Al}_{2} \mathrm{SiO}_{5}+\mathrm{Al}_{2} \mathrm{SiFl}_{10}$ könnte man nämlich annehmen, dass fünf andalusitartige Molekule, um ein Kernmolekül $\mathrm{Al}_{2} S i \mathrm{Sl}_{10}$ vereinigt, ein hemimorphes Topasmolekul bildeten. Dass Topas eine complicirte molekulare Constitution hat, zeigen auch die bedeutenden sowohl krystallographischen wie optischen Anomalien, welche Grunhut, Des Gloizeaux, Mallard, Friedel u. A. beschrieben haben.

Eine ganz andere Molekularconstitution muss für den mit Topas krystallographisch ähnlichen, aber chemisch unähnlichen D a nburit angenommen werden, dessen morphotropische Verwandtschaft mit Leuk oph a n, Melinophan und Skapolith schon oben (Abth. I) dargelegt worden ist. Dass auch in chemischer Hinsicht diese Mineralien verwandt sind, zeigt folgende Zusammenstellung, worin die krystallochemischen Constitutionsformeln für Leukophan und Melinophan aus den von Brögger (l. c.) gegebenen empirischen Formeln hergeleitet sind, und die fur Skapolith (mit $\mathrm{SiO}_{2}=\mathbf{4 0}$ $-50 \%$ ) mit den von Rammelsberg angenommenen Formeln übereinstimmen. Ich ziehe diese den von T schermak gegebenen Formeln vor, weil sie sich besser den übrigen Silicaten der genannten morphotropischen Reihe 
anschliessen, und weil $R$ a mmel sberg auch den von Dan a sogenannten Paranthin von Pargas aufnimmt, welcher nach den Analysen von N. Nordenskiöld eine mit Anorthit isomere oder polymere Abart des Skapoliths ist; dieses zeigen auch meine Analysen eines Skapolith von Helsingfors (Finska Vet. Soc. „Bidrag “ 46) und des oben genannten Anorthoits von Sillböle. Auch Dölter (Chem. Mineralogie 1890) sagt, dass ohne Zweifel ein Skapolith mit der ehemischen Zusammensetzung des Anorthits existirt. Die Skapolithe mit höherem $\mathrm{SiO}_{2}$-Gehalt als $50 \%$ habe ich hier nicht berücksichtigt. Wahrscheinlich enthalten sie, wie der grössere $\mathrm{Na}$-Gehalt zeigt, eine Verbindung von der Zusammensetzung des Albits, welche in der Skapolithgruppe gleichwie in der des Plagioklas isomorph mit $\mathrm{CaAl}_{2} \mathrm{Si}_{2} \mathrm{O}_{\mathrm{y}}$ ist.

$$
\begin{aligned}
& \text { Danburit } \quad 2\left(\mathrm{CaB}_{2} \mathrm{Si}_{2} \mathrm{O}_{8}\right) \\
& \text { Leukophan } \quad 2\left(\mathrm{CaBe}_{3} \mathrm{Si}_{2} \mathrm{O}_{8}+2 \mathrm{CaSi}_{2} \mathrm{O}_{5}+3 \mathrm{NaFl}\right) \\
& \text { Melinophan } 2\left(\mathrm{Na}_{2} \mathrm{Be}_{3} \mathrm{Si}_{2} \mathrm{O}_{8}+4 \mathrm{CaSiO}_{3}+\mathrm{BeFl}_{2}\right) \\
& \text { Skapolith } \begin{cases}\text { Paranthin } & 4\left(\mathrm{CaAl}_{2} \mathrm{Si}_{2} \mathrm{O}_{8}\right) \\
\text { Mejonit } & 4\left(\mathrm{CaAl}_{2} \mathrm{Si}_{2} \mathrm{O}_{8}\right)+\mathrm{Ca}_{2} \mathrm{SiO}_{4} \\
\text { Wernerit } & 4\left\{\begin{array}{ll}
\mathrm{CaAl}_{2} \mathrm{Si}_{2} \mathrm{O}_{8} \\
\mathrm{Na}_{2} \mathrm{Al}_{2} \mathrm{Si}_{2} \mathrm{O}_{8}
\end{array}\right\}+2 \mathrm{CaSi} \mathrm{O}_{2} \mathrm{O}_{5} .\end{cases}
\end{aligned}
$$

Wir haben in den Molekularcomplexen dieser Silicate ein Hauptmolekül von den Neben- resp. Kernmolekülen zu unterscheiden, jenes mit Anorthit, diese mit Wollastonit ähnlich zusammengesetzt, von welchen jedoch die letzteren in den Molekularcomplexen des Danburits und Paranthits vermisst werden, während die in einigen Skapolithvarietäten (Wernerit) als Kernmolekül vorkommende $\mathrm{NaCl}$-Verbindung dem $\mathrm{NaFl}$ im Leukophan entspricht. In dem Grundmolekul des Danburits kann man in Analogie mit dem der Pyroxenarten $\mathbf{2 B}_{2}=a$ und $\bar{a}\left(A l_{2}\right.$ im Pyroxen entsprechend), $2 C a=b$ und $\bar{b}, 4 S i=$ den vier Pyramidennormalen setzen, wodurch eine hemimorph-rhombische Molekularform gebildet wird. Fur Leukophan und Melinophan haben wir dagegen, den krystallographischen und chemischen Verbältnissen gemäss, drei Partialmolekule anzunehmen : ein Hauptmolekül ähnlich dem des Danburits, aber mit $\stackrel{\mathrm{BI}}{\boldsymbol{B}} e_{3}$ an Stelle von $\stackrel{\mathrm{III}}{\mathrm{B} o_{2}}$ und die SiAtome nicht einseitig hemimorph, sondern hemiëdrisch-sphenoidal gestellt, den abwechselnden vier Pyramidenflächen entsprechend, und zwei damit homöomorphe Nebenmoleküle an jedem Ende seiner Hauptaxe, ebenso mit den $\mathrm{Si}$-Atomen sphenoidisch angeordnet. Von den drei $\mathrm{Na}_{2} \mathrm{Fl}_{2}$-Molekülen kann eines als Kernmolekul in dem Hauptmolekull, die zwei anderen resp. die zwei $\mathrm{BeFl}_{2}$-Molekule im Melinophan, als verbindende Glieder (Zwischenmoleküle) zwischen diesem und den zwei Nebenmolekülen betrachtet werden. Hierbei kann noch bemerkt werden, dass in den zwei Nebenmolekulen des Leukophans nur zwei, in den des Melinophans vier $\mathrm{Ca}$-Atome, vier 
gleichen Normalen ( $a=b$ ) entsprechend, vorkommen, was die grössere Annäherung dieses letzteren zu dem tetragonalen Systeme erklärt. Uebrigens stehen die rhombischen Nebenmoleküle zu dem monoklinen Wollastonit in demselben Verhällnisse wie das Hauptmolekül zum Spodumen, d. $h$. einem Zwillingscomplex von diesem entsprechend oder einem Vierlingscomplex von Anorthit, von welchem der mit Danburit wahrscheinlich isomorphe Barsow it als eine polymere Modification betrachtet werden kann.

Fur die Grundform des Skapoliths kommen wir, in Hinsicht der Analogie sowohl mit den genannten morphotropen Silicaten als mit dem Zwillingscomplex des Anorthits (Fig. 5) zu der Annahme, dass die Si-Atome den Pyramidenflächen, die $A l_{2}$ - und $C a$-Atome resp. den Deutero- und Protoprismenflächen entsprechen, indem, wie oben (Ablh. II) gezeigt worden ist, die reciproken Werthe der Normalen $a b: a b c=1: 2=6: 12(C a: S i)$.

Dagegen sind im Vesuvian, den oben angedeuteten entgegengesetzten Formverhältnissen des Vesuvians und Skapoliths gemäss $(a b: a b c$ für Skapolith $=a: a c$ fur Vesuvian), die $C a$-Atome $=a$ resp. $b$ (die Normale des Deuteroprismas), die $S i$-Atome $=a c$ resp. $b c$ (die Normale der Deuteropyramide). Die $A l_{2}$-Atome entsprechen dann den intermediären Protoprismenrichtungen, uben aber, wie es scheint, keinen Einfluss auf die äussere Form aus, indem sie im Vesuvian wie im Skapolith die einzelnen Partialmolekulle zusammenhalten. Diese entgegengesetzte oder verwendete Stellung der Molekularformen des Skapoliths und Vesuvians ist analog mit der zwischen Spodumen resp. Alkalipyroxen und $\mathrm{Ca}$-Pyroxen oder zwischen Biotit und Sprödglimmer. Weiter zeigen die obigen Vergleichungen der Grundformen des Skapoliths und Vesuvians mit einem Zwillingscomplex des Anorthits, dass der mit der Skapolithform in seinen Winkeln analoge Vierlingscomplex von Plagioklas aus zwei Hälften, der der Vesuvianform ähnliche nur aus der einen Hälfte der Anorthoitform (Fig. 2) zusammengesetzt ist, und dass also der alkalihaltige Skapolith zu dem alkalifreien Vesuvian sich verhält, wie Alkalifeldspath zu Calciumfeldspath oder wie Spodumen zu dem alkalifreien Pyroxen.

Uebrigens muss der Vesuvian, wie zum Theil auch der Skapolith, aus zwei isomorphen Verbindungen bestehen, indem die von $R$ a mmelsberg gegebenen empirischen Formeln für Vesuvian auf folgende Weise ausgedrückt werden können :

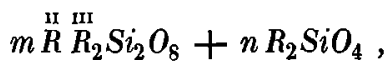

worin $m: n=1: 1,2: 3,4: 7$, also zwischen 1:1 und 1:2 angenommen werden kann. Diese zwei isomorphen Molekïle können entweder nebeneinander zusammenkrystallisiren, oder das aluminiumfreie kann als Kernmolektul zwischen zwei hemimorphen Complexen der aluminiumbaltigen Verbindung vorkommen. Diese verschiedenen Verhältnisse erklären die 
Variationen der Winkel der verschiedenen Vesuvianarten, wie die Ungleichheit der Winkel derselben Form durch die Zusammensetzung des Molekularcomplexes des Vesuvians aus weniger symmetrischen Partialmolekülen in molekularer Zwillingsverwachsung erklärt wird. Dass jedoch der Vesuvian, wie anomale Krystalle im Allgemeinen, bei höherer Temperatur normal tetragonal gewesen ist, zeigen die von Prendel gemachten Versuche mit Wiluit, dessen optische Zweiaxigkeit bei Temperaturerhöhung vermindert wird.

Wie schon oben angedeutet ist, steht der Leucit zu Skapolith in demselben Verhältnisse wie G r a a t , besonders der sehr anomale, in aufgewachsenen Krystallen ( $\infty 0 . \infty 02)$ vorkommende Granat von Pitkäranta, zu Vesuvian. Der Granat $\left(R_{3} R_{2} S_{3} O_{12}\right)$ zeigt dieselbe Zusammensetzung wie das Verhältniss. $m: n=1: 1$ in der des Vesuvians; der Leucit $\left(K \alpha_{2} A l_{2} S i_{4} O_{12}\right)$ verhält sich in quantitativer Beziehung zu Paranthin wie Andesin zu Anorthit, und kann in Rucksicht hierauf $=\mathrm{Ka}_{2} \mathrm{Al}_{2} \mathrm{Si}_{6} \mathrm{O}_{16}+\mathrm{Ka}_{2} \mathrm{Al}_{2} \mathrm{Si}_{2} \mathrm{O}_{8}=$ $2\left(\mathrm{Ka}_{2} \mathrm{Al}_{2} \mathrm{Si}_{2} \mathrm{O}_{8}+\mathrm{Si}_{2} \mathrm{O}_{4}\right)$ gesetzt werden. Nach der oben gemachten Vergleichung zwischen der Ikositetraëderform des Leucits und dem dieser entsprechenden Zwillingcomplexe des Plagioklases muss man ein Leucitmolekul betrachten als bestehend aus sechs hemimorphen pseudotetragonalen Molekularcomplexen (Fig. 5 der Tafel), also aus 24 quasi-monoklinen $\left(24 \mathrm{Ka}_{2} \mathrm{Al}_{2} \mathrm{Si}_{2} \mathrm{O}_{8}+\mathrm{Si}_{2} \mathrm{O}_{4}\right)$, jeder einem Bavenoer $\mathrm{Zwilling} \mathrm{der} \mathrm{Anorthitform}$ (Fig. 2) ähnlich, und folglich aus 48 asymmetrischen Partialmolekulen, deren (den Si-Atomen entsprechenden) Prismenflächen den Ikositetraëderflächen von Leucit, oder den damit vicinalen Hexakisoktaëderflächen gleichkommen. In diesem anomalen Zustande entspricht der Leucit einem anomalen Orthoklas (Anorthoklas). Ja es scheint sehr wahrscheinlich, dass der Leucit bei gewöhnlicher Temperatur nichts anderes ist, als eine Feldspathart von der Zusammensetzung des Hyalophans oder Andesins, welcher bei höherer Temperatur in eine polymere Modification davon übergeht.

Der Uebergang zwischen dem anomalen Leucit und dem anomalen Granat bilden die genannten anomalen aufgewachsenen Granatkrystalle von Pitkäranta, welche sich durch Tetrakishexaëderflächen vicinal zu $\infty 0$, von der allgemeinen Formel $\infty O \frac{m}{m-1}$, im Mittel $=\infty 0 \frac{41}{40}$, auszeichnen. Durch Untersuchungen von Platten, den Hexaëder- und Oktaëderflächen parallel, im polarisirten Lichte habe ich gefunden, dass diese Krystalle ein inneres Skelett von scharf abgesonderten, asymmetrischen Zwillingslamellen mit dem Auslöschungswinkel $15^{0}$ und den rhombendodekaëdrischen Pseudosymmetrieebenen parallel gehend, besitzen. Die Krystallräume zwischen diesen Lamellensystemen zeigen meistens Aggregatpolarisation; nur die parallel mit der Aufwachsungsebene gehende bexaëdrische Platte zeigt sich in der Mitte apolar und verhält sich also der basischen Ebene eines tetragonalen 
Krystalles ähnlich, während das Tetrakishexaëder $(\infty O 2)$, dessen Flächen nach A r z r u $\mathrm{n}$ i als Zwillingsebenen auftrelen, zum Theil den der Deuteropyramide $P \infty$ gleichkommen.

Der anomale dodekaëdrische Granat (Melanit) von Pitkäranta hat also eine Ausbildung analog mit der des Vesuvians und wohl auch eine mit diesem analoge Molekularstructur. Dagegen zeigt der mit Wiluit zusammen vorkommende ikosiletraëdrische Grossular ein weniger scharfes, an das des Leucits erinnerndes Lamellensystem, den Dodekaëderflächen und also wohl auch den Domenflächen des Zwillingscomplexes von Plagioklas parallel gehend, während in dem genannten dodekaëdrischen Melanit die Zwillingslamellen den pinakoidalen Zwillingsebenen dieses Zwillingscomplexes entsprechen.

Von diesen anomalen Granatvarietäten bis zu dem vollkommen normalen in Granit eingewachsenen Eisenaluminiumgranat (Almandin) findet sich nun eine Menge von Klein, Mallard u. A. beschriebener Uebergänge, welche jedoch alle auf einer grösseren oder kleineren Veränderung der ursprünglichen Atom- resp. Molekular-Anordnung beruhen müssen. Die bei Temperaturveränderung bewirkte innere Spannung oder Druck, wie auch die von Brauns beobachtete Anomalie durch Zusammenlagerung homöomorpher Molekule können dabei nur als mitwirkende Nebenursachen betrachtet werden. Dass der anomale Granat nicht wie Leucit bei Temperäurerhöhung in den ursprünglichen normalen Zustand übergeht, beruht wohl auf den verschiedenen Elementarbestandtheilen der beiden, indem die alkalibaltigen Verbindungen im Allgemeinen leichter als die alkalifreien aus dem einen in den anderen Zustand überzugehen scheinen, was eine grössere Beweglichkeit oder geringere Trägheit (Indifferenz) der Moleküle jener im Vergleich mit diesen anzeigt. Die grössere Mannigfaltigkeit der anomalen Molekularstructur des Granats als derjenigen des Leucits erklärt sich dadurch, dass der Granat nicht nur aus pseudotetragonalen, sondern auch, gleichwie der Sodalith, aus pseudohexagonalen Molekularcomplexen aufgebaut ist, und jeder von diesen sich in rhombische mono- und asymmetrische Partialmoleküle sondern kann.

Zu den Silicaten, welche in ihren Prismenzonen mit der brachydiagonalen (a-) Zone des Bavenoer Zwillingscomplexes von Plagioklas ubereinstimmen, gehören auch die Z eolithe, welche sowohl krystallographisch wie chemisch sich den Plagioklasen anschliessen, indem sie sich auf ähnliche homologe Reihen zurückführen lassen, wie Streng, Fresenius und Rammelsberg gezeigt haben. So bildet die Desmingruppe folgende homologe Reihe:

$$
\begin{aligned}
& \text { Des } \mathrm{min} \\
& \stackrel{\mathrm{II}}{\mathrm{R} A l_{2}} \mathrm{Si}_{6} \mathrm{O}_{16}+6 \mathrm{H}_{2} \mathrm{O} ; \quad\left(\stackrel{\mathrm{II}}{\mathrm{R}}=\mathrm{Ca}, \mathrm{Na}_{2}\right) \\
& \text { Harmotom } \quad \stackrel{\mathrm{II}}{\mathrm{R} A l_{2}} \mathrm{Si}_{5} \mathrm{O}_{14}+5 \mathrm{H}_{2} \mathrm{O} ; \quad\left(\stackrel{\mathrm{I}}{\boldsymbol{R}}=\mathrm{B} a, K a_{2}\right) \\
& \text { Phillipsit } \quad \stackrel{\text { II }}{R} \mathrm{Al}_{2} \mathrm{Si}_{4} \mathrm{O}_{12}+4 \mathrm{H}_{2} \mathrm{O} ; \quad\left(\stackrel{\mathrm{II}}{\mathrm{R}}=\mathrm{Ca}, \mathrm{Ka} a_{2}, \mathrm{Na} a_{2}\right) \text {. }
\end{aligned}
$$


Da aber nach den Untersuchungen von Damour (siehe Rammelsberg, Mineralchemie 2) im Desmin 2 und im Harmotom $1 \mathrm{Mol}$. $\mathrm{H}_{2} \mathrm{O}$ als Constilutionswasser" (Hydroxyl) betrachtet werden muss, so erhalten diese isomorphen Zeolithe eine gleiche Anzahl Krystallwassermoleküle. Diese müssen in dem holomorphen physikalischen Molekül $=8$ gesetzt werden, indem der Phillipsit in Uebereinstimmung mit dem entsprechenden Glied der Plagioklasreihe $=\left(\stackrel{\mathrm{II}}{R} A l_{2} S i_{6} O_{16}+6 \mathrm{aq}\right)+\left(\stackrel{\mathrm{II}}{R} A l_{2} S i_{2} \mathrm{O}_{8}+2 \mathrm{aq}\right)=2\left(\stackrel{\mathrm{Ir}}{R} A l_{2} \mathrm{Si}_{4} \mathrm{O}_{12}\right.$ $+4 \mathrm{aq})$ ist. In Uebereinstimmung hiermit und in Analogie mit frtiheren ähnlichen Fällen in der Feldspath-, Glimmer- u. a. Gruppen kann man dann annehmen, dass im Desmin 2 (resp. 4) und im Harmolom 1 (resp. 2) Mol. $\mathrm{SiO}_{2}$ nebst den genannten Hydroxylmolekülen innerbalb des Molekularcomplexes auftreten, und dass mit Hinsicht auf die oben (Abth. I) angeführte Analogie zwischen dem Formencomplex des Desmins und einem Plagioklaszwillingscomplex 4 (resp. 8) Si-Atome den Pyramidenflächen des rhombischen Complexes oder den Prismenflächen der monoklinen Partialformen und die $\mathrm{Ca}$ - und $\mathrm{Al}_{2}$-Atome den Pinakoidflächen entsprechen.

An diese homologen Reihen schliessen sich die rhombischen resp. monoklinen, pseudotetragonalen Mineralien Gismondin und $\mathrm{Zeagonit}$ mit resp. 3 und 2 Molekülen $\mathrm{SiO}_{2}$, aber mit constantem Wassergehalt (4 Mol. $\left.\mathrm{H}_{2} \mathrm{O}\right)$ an. Dagegen hat der weniger symmetrische, mono- resp. trikline Heulandit eine ungerade Anzahl von Wassermolekülen, 5 oder 3 , je nachdem man die gesammte Anzahl oder nur die von Damour und Rammelsberg als Krystallwasser zu betrachtenden Molekule berucksichtigt. Da nun ubrigens der Heulandit analog mit Desmin zusammengesetzt ist, muss wohl die verschiedene Krystallform dem ungleichen Krystallwassergehalt zugeschrieben werden, was auch durch die Veränderung des Heulanditkrystalles in optischer Hinsicht beim Fortgehen der drei Krystallwassermolekitle angezeigt wird; nach den Untersuchungen von Rinne kann diese Veränderung als eine Annäherung zum rhombischen Systeme gedeutet werden. Eine solche Veränderung habe ich auch bei dem zwischem Desmin und Heulandit stehenden Epistilbit aus Island beobachtet, woraus geschlossen werden kann, dass der Epistilbitkrystall bei höherer Temperatur normal rhombisch gewesen ist, wie wahrscheinlich auch der Desmin, aber bei niederer Temperatur in zwei monokline, mit Heulandit analoge Partialformen getheilt worden ist.

In der Gruppe der Nadelzeolithe verbält sich der monokline S k o lezit $\left(\mathrm{CaAl}_{2} \mathrm{Si}_{3} \mathrm{O}_{10}+3 \mathrm{H}_{2} \mathrm{O}\right)$ zu. dem rhombischen $\mathrm{Natrolith}\left(\mathrm{Na}_{2} \mathrm{Al}_{2} \mathrm{Si}_{3} \mathrm{O}_{10}+\right.$ $\left.2 \mathrm{H}_{2} \mathrm{O}\right)$, wie der Heulandit zum Desmin in der Gruppe der Blätterzeolithe, d. h. das Molekularverhältniss zwischen dem Skolezit und dem Natrolith ist $=1: 2$ oder gleich dem Verhältnisse zwischen Anorthit und Albit. Da nun der Skolezit gleich 


$$
\left(\mathrm{CaAl} \mathrm{Si}_{2} \mathrm{O}_{16}+6 \mathrm{aq}\right)+3\left(\mathrm{CaAl}_{2} \mathrm{Si}_{2} \mathrm{O}_{8}+2 \mathrm{aq}\right)=4\left(\mathrm{Ca} A \mathrm{l}_{2} \mathrm{Si}_{3} \mathrm{O}_{10}+3 \mathrm{aq}\right)
$$

gesetzt werden kann, so ist das Skolezitmolekül gleich vier, das des Natroliths gleich acht hemiëdrisch-triklinen plagioklasähnlichen Partialmolekülen $\left(\mathrm{RAl}_{2} \mathrm{Si}_{2} \mathrm{O}_{8}+\mathrm{SiO}_{2}\right)$ mit 12 resp. 8 Molekülen Krystallwasser zu setzen, worauf auch, wie oben (Abth. I) gezeigt worden ist, die Krystallformen dieser Zeolithe hinweisen. Diese Vergleichung der genannten Formen mit den entsprechenden Zwillingsformen des Plagioklases zeigt weiter, dass die Grundformen des Desmins und Natroliths in verwendeter Stellung zu einander stehen, indem die Prismen- und Domenflächen dieses letzteren den Pinakoid- und Pyramidenflächen jenes krystallochemisch gleichkommen.

Die in die oben angenommene Zusammensetzung fur Natrolith und Skolezit eingehende Verbindung $\mathrm{RAl}_{2} \mathrm{Si}_{2} \mathrm{O}_{8}+2$ aq bildet in Form eines Doppelmoleküls mit $(\mathrm{HO})_{2}$ als verbindendem Kernmolekul das Grundmolekul des Thomsonits, dessen Axenverbältniss auch auf ein solches hinweist, indem seine $c$-Axe $=0,714=2 \cdot 0,352$ oder zweimal der Hauptaxe des Natroliths ist.

Auch der La umont it schliesst sich in morphologischer Beziehung den Nadelzeolithen an, obgleich seine empirische Formel gleich der des Phillipsits ist, wobei jedoch nur die Hälfte des Wassergehaltes oder 2 Mol. $\mathrm{H}_{2} \mathrm{O}$ als Krystallwasser zu betrachten ist, welches der Monosymmetrie des Laumontits im Gegensatze zur Disymmetrie des Phillipsits entspricht. Krystallochemisch kann der Laumontit mit dem Pyroxen verglichen werden, und verhält sich zum Analcim wie Partschin zum Granat.

Wie die Zeolithe der Desmin- und Natrolithgruppe können auch die der Chabasitgruppe sowohl in Hinsicht ihrer chemischen wie krystallographischen Verhältnisse als Verbindungen von wasserhalligen Plagioklasen, welche sich in zwei homologe Reihen anordnen lassen, belrachtet werden, die eine mit Grenzgliedern mit resp. 8 und $4 \mathrm{Mol} . \mathrm{H}_{2} \mathrm{O}$, die andere mit 7 und 3 Molekülen. Doch kommen nicht die Grenz- sondern die Mittelglieder und vorzugsweise die mit $6 \mathrm{Mol} . \mathrm{H}_{2} \mathrm{O}$ vor. Man kann also annehmen, dass wie die pseudo- resp. mimetisch-tetragonalen Zeolithe Molekularcomplexe mit 4 Mol. Krystallwasser oder Multipla davon enthalten, so die mimetischbexagonalen 6 Moleküle, theils mit, theils ohne Hydroxyl als Kernmolekül.

Das A pophyllit-Molekul kann man sowohl mit Hinsicht der oben angefuhrten krystallographischen und optischen Verhältnisse wie auch seiner chemischen Zusammensetzung (nach Rammelsberg) betrachten als bestehend aus vier rhombischen $\mathrm{Ok}$ enit-Molekulen um ein Mol. KaFl (nebst Hydroxyl) als Kernmolekül :

$$
\begin{array}{ll}
\text { Okenit } & \mathrm{H}_{2} \mathrm{CaSi}_{2} \mathrm{O}_{6}+\mathrm{aq}, \\
\text { Apophyllit } & 4\left(\mathrm{H}_{2} \mathrm{CaSi}_{2} \mathrm{O}_{6}+\mathrm{aq}\right)+\mathrm{KaFl} .
\end{array}
$$

Der Prismenwinkel des Okenits $\left(=5^{\circ}\right)$ ist nämlich nahe gleich dem 
Mittelkantenwinkel dẹ Pyramide $\left(60^{\circ} \longrightarrow 59^{0}\right)$ des Apophyllits; und nach oben angenommener Atomlagerung von Anorthit, Wollastonit und Margarit kann für den Okenit $\mathrm{Ca}=b, \mathrm{Si}=a b$ gesetzt werden, und in Folge davon für Apophyllit $C a=c, S i=a b c$.

Der Analcim schliesst sich krystallochemisch dem Leucit an. Dass eine nahe Analogie ihrer Molekularstructur vorliegt, zeigt der von Lemberg nachgewiesene Uebergang des einen in den anderen. Im polarisirten Lichte zeigen die kleineren, z. B. die von Schultén gemachten Analcimkrystalle eine analoge Theilung in scheinbar rhombische Theile, wie kleinere Leucitkrystalle, wogegen grössere Krystalle, wie die des in Süd-Norwegen vorkommenden Eudnophits (nach Brögger l. c.) Zwillingslamellen nicht nur parallel mit den Rhombendodekaëder-, sondern auch mit den Hexaëderflächen zeigen, und diese schärfer als jene, in Uebereinstimmung mit dem Plagioklaszwillingscomplex in der fur die Vergleichung mit Leucit angenommenen Stellung (siehe Fig. 6), so dass die Flächen des Hexaëders den Pinakoidflächen, die des Rhombendodekaëders den Brachydomenflächen und die des Ikositetraëders den Prismenflächen des Plagioklascomplexes entsprechen.

Die dritte Abtheilung der Silicate oder diejenige, welche in einer Hauptzone Aehnlichkeit mit der rhombisch-monoklinen $b$-Zone der Feldspatharten zeigen; können jedoch nicht so direct mit diesen verglichen werden, wie die meisten vorher angeführten; und es ist darum schwieriger, in eine nähere Betrachtung ihrer Molekularconstitution einzugehen. Sie können daher hier nur in grösserer Allgemeinheit betrachtet werden.

Die schon oben angezeigte krystallographische Aehnlichkeit dieser Silicate steht mit einer entsprechenden chemischen im Zusammenhange, wie folgende Zusammensetzung zeigt :

$\begin{array}{ll}\text { Prebnit } & \mathrm{H}_{2} \mathrm{Ca}_{2} \mathrm{Al}_{2} \mathrm{Si}_{3} \mathrm{O}_{12}, \\ \text { Datolith } & \mathrm{H}_{2} \mathrm{Ca}_{2} \mathrm{~B}_{2} \mathrm{Si}_{2} \mathrm{O}_{10}, \\ \text { Homilit } & \mathrm{FeCa}_{2} \mathrm{~B}_{2} \mathrm{Si}_{2} \mathrm{O}_{10}, \\ \text { Gadolinit } & \mathrm{FeBe}_{2} \mathrm{Y}_{2} \mathrm{Si}_{2} \mathrm{O}_{10}, \\ \text { Euklas } & \mathrm{H}_{2} \mathrm{Be}_{2} \mathrm{Al}_{2} \mathrm{Si}_{2} \mathrm{O}_{10}, \\ \text { Zoisit } & \mathrm{H}_{2} \mathrm{Ca}_{2} \mathrm{Al}_{2} \mathrm{Si}_{2} \mathrm{O}_{10}+2 \mathrm{CaAl}_{2} \mathrm{Si}_{2} \mathrm{O}_{8}, \\ \text { Epidot } & \mathrm{H}_{2} \mathrm{Ca}_{2}\left(\mathrm{Al}_{2}, \mathrm{Fe}_{2}\right) \mathrm{Si}_{2} \mathrm{O}_{10}+2 \mathrm{CaAl} \mathrm{Si}_{2} \mathrm{O}_{8}, \\ \text { Orthit } & \mathrm{H}_{2}(\mathrm{Ca}, \mathrm{Fe})_{2}\left(\mathrm{Al}_{2}, \mathrm{Ce}_{2}, \mathrm{Fe}_{2}\right) \mathrm{Si}_{2} \mathrm{O}_{10}+\mathrm{CaAl}_{2} \mathrm{Si}_{2} \mathrm{O}_{3} .\end{array}$

Auch die Zusammensetzung des Johnstrupits resp. Mosandrits scheint sich dieser Reihe, am nächsten dem Orthit anzuschliessen, jedoch mit einem einfachen $\mathrm{Ca}$-Silicat an Stelle des $\mathrm{CaAl}$-Silicats.

Der Homologie des Prehnits und Datoliths wird von ibren oben angefuhrten krystallographischen Verhältnissen entsprochen, wenn man sich das Prehnitmolekul gleich zwei Datolithmolekülen durch ein Kernmolekul 
von $2 \mathrm{SiO}_{2}$ verbunden denkl. Uebrigens kann man bei der Betrachlung der Molekularverhältnisse dieser Silicate von dem Epidot ausgehen, welcher nach obiger Auffassung aus $2 \mathrm{Mol}$. einer mit Anorthit analogen Verbindung $\left(\mathrm{CaAl}_{2} \mathrm{Si}_{2} \mathrm{O}_{8}\right)$ und $1 \mathrm{Mol} . \mathrm{H}_{2} \mathrm{Ca}_{2} \mathrm{Al}_{2} \mathrm{Si}_{2} \mathrm{O}_{10}$ resp. $\mathrm{H}_{2} \mathrm{Ca}_{2} \mathrm{Fe}_{2} \mathrm{Si}_{2} \mathrm{O}_{10}$ besteht. Diese Auffassung wird durch die Analysen bestätigt, indem nach diesen das Maximum des $\mathrm{Fe}_{2} \mathrm{O}_{3}$-Gehaltes $=17 \%$ ist. Nach dieser Ansicht sind die Epidotvarietäten Verbindungen von einem $\mathrm{Al}_{2} \mathrm{O}_{3}$-Epidot von der Zusammensetzung des Zoisits und einem $\mathrm{Fe}_{2} \mathrm{O}_{3}$-Epidot, dessen berechnete Zusammensetzung nachstehend mit einem Epidot von Arendal (nach Scheerer) zusammengestellt ist.

\begin{tabular}{ccc} 
& Eisen-Epidot: & Epidot von Arendal : \\
$6 \mathrm{SiO}_{2}$ & 37,27 & 37,59 \\
$2 \mathrm{Al}_{2} \mathrm{O}_{3}$ & 21,12 & 20,73 \\
$\mathrm{Fe}_{2} \mathrm{O}_{3}$ & 16,57 & 16,57 \\
$4 \mathrm{CaO}$ & 23,18 & $22,64(\mathrm{MgO}=0,41)$ \\
$\mathrm{H}_{2} \mathrm{O}$ & 1,86 & 2,11 \\
\cline { 2 - 3 } & 100,00 & 100,00
\end{tabular}

Diese Auffassung steht auch mit den oben (Abth. J) angefuhrten krystallographischen Verhältnissen des Epidots in Uebereinstimmung, wonach das Epidotmolekúl durch die längere Diagonalebene der Grundform, welche dem negativen Doma $(-\mathbb{\infty})$ parallel gebt, in zwei ungleiche Theile getheilt:werden kann : der eine Theil aus zwei hemimorphen Partialmolekulen $2 \mathrm{CaAl}_{2} \mathrm{Si}_{2} \mathrm{O}_{8}$, der andere aus $\mathrm{Ca}_{2} \mathrm{Al}_{2} \mathrm{Si}_{2} \mathrm{O}_{9}$ resp. $\mathrm{Ca}_{2} \mathrm{Fe}_{2} \mathrm{Si}_{2} \mathrm{O}_{9}$ bestehend und durch ein Kernmolekul $\mathrm{H}_{2} \mathrm{O}$ mit dem vorigen verbunden. Diese zwei Theile verhalten sich zu einander wie die zwei Theile der Molekularform des Albits in der Richtung der Brachydiagonale, und wie man von jenen durch Zwillingsbildung zuerst einen pseudomonoklinen, dann einen pseudorhombischen Formencomplex erbält, so kommt man auch von der Epidotform, wie oben angedeutet ist, zu den monoklinen und rhombischen Formen der Datolith-Zoisitreihe. In Uebereinstimmung hiermit sind die Molekularformen des Datoliths, Homilits und Gadolinits als hemiëdrisch-monoklin analog mit denen des Kaolins und Pyroxens zu betrachten, worauf auch das von Brögger (diese Zeitschr. 16, 147) angegebene Verhältniss zwischen Gadolinit und Homilit (resp. Datolith), dass die positive Seite jenes der negativen Seite dieses entspricht, hinzudeuten scheint. Uebrigens zeigt die von Brögger gefundene "Stundenglasstructur " des Homilits eine molekulare Zwillingsverwachsung an mit den $a$ - und $c$-Flächen, (100) und (001), als $\mathrm{Zwillingsebenen,} \mathrm{und} \mathrm{so} \mathrm{zusammengesetzt,} \mathrm{dass} \mathrm{an} \mathrm{einem} \mathrm{centralen}$ Zwillingscomplex einfache Molekule sich symmetrisch anlagern.

Nach den oben (Abth. II) angefuhrten reciproken Werthen der Normalen der Grundflächen am Epidot kann man $c=C a, a b$ und $a b c=S i$ setzen, und daneben annehmen, dass sie durch Kantenstellung die nahezu 
oktaëdrischen Winkel in den Prismen- und Pyramidenzonen bewirken. Die $\mathrm{Al}_{2^{-}}$(resp. $\mathrm{Fe}_{2_{2}}$ ) Alome könnte man der $a-(\operatorname{resp} . \bar{a}-)$ Fläche $(T)$ gleich selzen, da diese, wie oben gezeigt worden ist, der $c$-Fläche $(P)$ des Feldspaths entspricht. An dem Datolith verhält sich die Flächennormale der gewöhnlichsten Pyramide $-\mathbb{R 2}\left(=\sin 29^{\circ} 31^{\prime}\right)$ zu der Normale des Klinopinakoids $(b-\mathrm{Axe})=0,49: 1$ oder nahe gleich $0,5: 1$, und also die reciproken Werthe $a b_{2} c_{2}: b_{1}=2: 1$ oder wie $a b_{1}: c_{1}(=S i: C a)$ am Epidot. Dieses führt für Datolith zur Annahme einer mit der fur Kaolin (9) ähnlichen Molekularformel mit $C a$ an Stelle des Wasserstoffes und $\boldsymbol{B}_{2}$ an Stelle des Aluminiums. Die pinakoidale Stellung der Atome $C a$ und $B_{2}$ entspricht der normalen Ausbildung: $a b, a b_{2}, a_{2} b$ etc.

Diese Auffassung des Verbältnisses zwischen den genannlen monoklinen und rhombischen Silicaten lässt sich auch auf die krystallochemischen Verhältnisse zwischen Titanit und Liëvrit anwenden, welche Silicate (siehe oben Abth. I) in krystallochemischer Beziehung sich dem Epidot und seinen Verwandten anschliessen. Für Titanit erhält man folgendes Verhältniss der Normalen der Hauptflächen $r(110), n(221), l(\overline{1} 11)$ nach D a n a's Grundform:

$$
\begin{aligned}
& \vec{a} b^{1}=\sin 33^{015^{\prime}}=0,5483(3), \\
& \bar{a} b c^{1}=\sin 337=0,3918(2,14), \\
& a b c^{2}=\sin 2154=0,3730(2,04) .
\end{aligned}
$$

Man erbält also für Titanit dasselbe Verbältniss der reciproken Werthe der Normalen der genannten Prismen- und hemipyramidalen Flächen wie oben fur Pyroxen, was zu der Annahme fuhrt, dass $C a$ den Prismen-Normalen, $S i$ und $T i$ den Pyramiden-Normalen entsprechen, jenes in Kanten-, diese in Flächenstellung. Da nun weiter $n: n=43^{\circ} 48^{\prime}, l: l=46^{\circ} 8^{\prime}$, oder gleich den Hauptflächenwinkeln für Anatas $\left(43^{0} 24^{\prime}\right)$ und Quarz $\left(4^{\prime} 6^{0} 26^{\prime}\right)$ sind, kann man annehmen, dass die $n$-Flächen den $T l$-Atomen, die $l$-Flächen den $\mathrm{Si}$-Atomen entsprechen; und man erhält so fur Titanit $\left(\mathrm{Ca}_{2} \mathrm{Ti}_{2} \mathrm{Si}_{2} \mathrm{O}_{10}\right)$ eine analoge Molekularform, wie für einige fruher angefubrte monokline Silicate, nämlich ein aus zwei ungleichen Partialmolekưlen in der Richtung der Symmetrieebene bestehendes Molekül, das eine $C a(=\bar{a} b)$ und $S i(=\bar{a} b c)$, das andere $T i\left(=a_{2} b_{2} c\right)$ enthaltend. - Ein solches Ti-haltiges Partialmolekul ist wohl auch in dem von Brögger (l. c.) sogenannten Aịigmatit (Gossyrit Förstner's) anzunehmen, an ein triklines Babingtonitmolekul gefügt und ein Spaltungsprisma von $66^{\circ} 6^{\prime}$, gleich dem Winkel des Titanitprismas verursachend.

Auf Grund der oben angeführten krystallochemischen Beziehungen zwischen Liëvrit und Titanit kann man annehmen, dass sie in krystallochemischer Hinsicht zu einander in demselben Verhältnisse stehen, wie mehrere im Vorhergehenden angefuhrte, nahe verwandte rhombische und monokline Silicate, d. h. dass das Liëvritmolekül aus zwei quasi-monoklinen 
Parlialmolek tulen, der positiven, $\mathrm{CaSiO}_{3}$ enthaltenden Hälfte des Titanitmoleküls entsprechend, zusammengesetzt ist. Doch ist hierbei zu bemerken, dass Liëvrit eine normale Ausbildung in der Prismenzone zeigt, indem das Prisma $\infty \breve{P} 2$ dem Grundprisma $\infty P$ zunächst folgt, wäbrend am Titanit das Prisma $\infty R 3$ das gewöhnlichste secundäre Prisma ist. Dieses deutet auf eine pinakoidale (b-) Stellung für $C a$ im Liëvrit, im Gegensatze zur prismatischen im Tilanit (nach Dana's Grundform). Für die dreiatomigen Radicale $\mathrm{Al}_{2}, \mathrm{Y}_{2}$ und $\mathrm{Fe}_{2}$ in den Molekülen des Ytrotitanits und Liëvrits könnte man dann eine ähnliche Stellung wie in denjenigen mehrerer vorhergehender Silicate der zweiten und dritten Abtheilung, nämlich eine ortho- resp. makropinakoidale $a$-Slellung, annehmen, indem die Grundform des Liëvrits in der Richtung der $a$-Axe sich am meisten von der entsprechenden Richtung des Titanits unterscheidet. Doch scheinen $\mathrm{Al}_{2}$ und seine Stellvertreter hier wie in mehreren vorhergehenden ähnlichen Fällen weniger Einfluss auf die äussere Formen auszuuben als $C a$ und $S i$.

Auch zwischen Liëvrit und Epidot resp. Zoisit könnte man eine Beziehung finden, wenn man die Formel des Liëvrits auf folgende Weise schreibt:

$$
\mathrm{H}_{2} \mathrm{Ca}_{2} \mathrm{Fin}_{2} \mathrm{Si}_{2} \mathrm{O}_{10}+\mathrm{FeFe}_{3} \mathrm{Si}_{2} \mathrm{O}_{8} \text {, }
$$

indem der Liëvrit dann betrachtet werden kann als mit Epidot analog zusammengesetzt, aber mit einer Eisenolivinverbindung an Stelle der Anorthitverbindung des Epidots, was die von Websky angezeigte Analogie zwischen den Formen des Liëvrits und Humils erklären könnte.

Der Kentrolith, welcher in der Prismenzone sich den mit Epidot verwandten monoklinen und rhombischen Silicaten anschliesst, zeigt auch in seiner chemischen Zusammensetzung $\left(\mathrm{Mn}_{2} \mathrm{~Pb}_{2} \mathrm{Si}_{2} \mathrm{O}_{9}\right)$ Aehnlichkeit mit diesen, woraus geschlossen werden kann, dass das Constitutionswasser als Kernmolekul keinen oder wenig Einfluss auf die äussere Form ausübt.

Den epidotartigen Silicaten schliessen sich noch folgende an :

$$
\begin{array}{ll}
\text { Karpholith } & \mathrm{H}_{2}\left(\mathrm{H}_{2} \mathrm{Mn}\right) \mathrm{Al}_{2} \mathrm{Si}_{2} \mathrm{O}_{10} \\
\text { Ardennit } & \mathrm{5}\left(\mathrm{H}_{2} \mathrm{Mn}_{2} \mathrm{Al}_{2} \mathrm{Si}_{2} \mathrm{O}_{10}\right)+\mathrm{V}_{2} \mathrm{O}_{5} .
\end{array}
$$

Krystallographisch scheinen sich, so weit man aus ihren unvollkommenen Krystallen schliessen kann, der Karpholith den monoklinen, der Ardennit den rhombischen Silicaten der Epidot-Zoisitgruppe anzuschliessen. Obige Formel fur Ardennit deutet eine Molekularverbindung an aus fünf einzelnen Karpholithmolekủlen bestehend und durch das fünfatomige Vanadium ( $V_{2}$ resp. Vanadyl $V_{2} O_{2}$ ) als Kernmolekul zu einem Molekularcomplex vereinigt, ahnlich dem oben angeführten Molekularcomplex des Topases. Hieraus erklărt sich auch die Verschiedenheit in den Axenverhältnissen des Ardennits und Zoisils, indem nach vo m Rath $a: b: c$ (Ardennit) $=0,466$ : 1:0,313 oder nahe $=\frac{3}{2} a: 1: \frac{1}{2} c$ am Zoisit. Die deutliche Spaltbarkeit 
nach der $b$-Fläche $\left(\infty \breve{P}_{\infty}\right)$, also normal gegen die grösste Axe, und die säulenförmige Ausbildung in der Richtung der kleinsten oder $c$-Axe sprich für dieses Axenverhältniss als Ausdruck für die Grundform resp. das Grundmolekul des Ardennits.

Die Grundformen der Silicate der dritten Abtheilung oder die von der $b$-Zone der Feldspathformen derivirten unterscheiden sich von den regulären Formen im Allgemeinen mehr als die der zwei anderen Abtheilungen, wie ja auch die Feldspathformen in dieser Zone am meisten von den entsprechenden regulären Formen abweichen. Auch lassen sich Formen höherer Symmetrie, wie die in den vorigen Abtheilungen, von den Formen der dritten Abtheilung nicht deduciren.

Ich habe im Vorhergehenden vorzugsweise die kryslallographischen und chemischen Eigenschaften der Silicate betrachtet. Dass aber auch die optischen Erscheinungen sich in Uebereinstimmung mit den vorigen betrachten lassen, habe ich in mehreren Fällen gefunden. Wie die krystallographischen Symmetrieverhälnnisse sich durch die Annahme molekularer Zwillingsverwachsungen auf oben angeführte Weise erklären lassen, so kônnen auch im Allgemeinen die optischen Elasticitätsverhältnisse dadurch erklärt werden. Aber auch in mehreren speciellen Fällen gelangt man zu einer Uebereinstimmung zwischen den theorelischen und den auf empirischem Wege erhaltenen Daten, wenn man die bekannte Formel zur Berechnung des specifischen Brechungsvermögens und der Brechungsexponenten einer Verbindung aus denjenigen ihrer Bestandtheile auf die in der resp. Hauptrichtung der Grundform vertheilten Elemente anwendet, und dabei annimmt, dass die optische Elasticität auf den Aethersphären der resp. Elemenlaratome beruht. So z. B. kommt man von der oben für Skapolith deducirlen Atomlagerung, nach welchem $C a$ und $A l$ eine prismatische resp. pinakoidale, $S i$ eine pyramidale Stellung einnehmen, zu folgenden berechneten Werthen für die Brechungsexponenten des ordinären und extraordinären Strables :

$$
\begin{aligned}
& \mathrm{CaO}: \text { Refractionsäquivalent }=\mathbf{1 3 , 3} ; \text { Mol.-Gew. }=\mathbf{5 6} \\
& \mathrm{Al}_{2} \mathrm{O}_{3} \quad \text { - }=19,7 ; \quad-\quad=103 \\
& \mathrm{SiO}_{2} \quad-\quad=12,6 ;-\quad=60 \text {, }
\end{aligned}
$$

woraus das specifische Brechungsvermögen für

$$
\begin{aligned}
\mathrm{CaO}+\mathrm{Al}_{2} \mathrm{O}_{3} & =\frac{13,3+19,7}{56+103}=\frac{33}{159}=0,2075 \\
\mathrm{SiO}_{2} & =\frac{12,6}{60}=0,201 ;
\end{aligned}
$$

und also, wenn das spec. Gewicht für Skapolith $=2,7$ geselzt wird: 


$$
\begin{aligned}
& \omega=2,7 \cdot 0,208+1=1,56, \\
& \varepsilon=2,7 \cdot 0,201+1=1,54 .
\end{aligned}
$$

Diese Werthe stimmen mit den von Des Cloizeaux für Skapolith von Arendal gefundenen: $\omega=1,56-1,57, \varepsilon=1,542-1,543$ uberein.

Auf ähnliche Weise habe ich für Nephelin unler Annahme, dass $\mathrm{Na}_{2} \mathrm{O}+2 \mathrm{SiO}_{2}$ der ordinären, $\mathrm{Al}_{2} \mathrm{O}_{3}+3 \mathrm{SiO}_{2}$ der extraordinären Schwingungsrichtung entsprechen, $\omega=1,5444$ und $\varepsilon=1,5434$ erhalten, welche Werthe mit den von Ramsay am Eläolith von Iivaara (spec. Gew. = 2,63) gefundenen Brechungsexponenten $\omega=1,5451$ und $\varepsilon=1,5420$ ziemlich nahe ubereinstimmen. Ebenso erhält man, von der Annahme ausgehend, dass in braunem und schwarzem Turmalin $B_{2}+2 A l_{2}$, in rothem Alkaliturmalin $\mathrm{B}_{2}+2 \mathrm{Al}_{2}$ die Aetherelasticität des extraordinären Strables bewirken (das spec. Gew. $=3,1$ gesetzt) die Brechungsexponenten $\varepsilon=1,63$ und 1,61, oder gleich des von Jerofejew und Schwebel gefundenen Werthen $\varepsilon=1,63$ (für braunen und blauen Turmalin) und $\varepsilon=1,61$ (für rothen Turmalin vom Ural). Der Brechungsexponent des ordinären Strahles lässt sich wegen der variirenden Elemente in der Prismenzone nicht be-rechnen.

Auch bei den optisch-zweiaxigen Silicaten stehen im Allgemeinen die optischen Erscheinungen mit den oben erhaltenen theoretisch-krystallochemischen Verhältnissen in Uebereinstimmung; so z. B. ist die Richtung der grössten Elasticitätsaxe $(\alpha)$ nahe parallel mit den einander entsprechenden $a$ - und $c$-Axen am Ortboklas und Epidot, und mit der der $a$-Axe in den Zwillingscomplexen des Feldspaths entsprechenden $c$-Axe des Skapoliths. An den in krystallochemischer Hinsicht analogen Grundformen des Pyroxens und Titanits sind auch die optischen Elasticitätsaxen gleich vertheilt, indem die kleinste Axe in dem stumpfen, die grösste in dem spitzen Winkel derselben liegen. Die grosse Verschiedenheit der Brechungsexponenten dieser Elasticitätsrichtungen am Titanit entspricht der bedeutenden Verschiedenheit des Brechungsvermögens der Titan- und Kieselsäure (resp. 0,411 und 0,201), und der oben angenommenen Lagerung der Ti-Atome an den positiven (spitzen) Pyramidenseiten des Grundmoleküls.

Aus dem Verhältnisse, dass die metallischen Mineralien im Allgemeinen einen grösseren Brechungsexponenten zeigen, als die nichtmetallischen, kann man schliessen, dass die schweren Metalle eine geringere optische Elasticität oder ätherische Kraft haben, als die leichten Metalle resp. Metalloide; und in Uebereinstimmung hiermit findet man auch, dass meistentheils diejenigen Richtungen der Grundform, in welchen nach der Theorie die basischen (positiven), schweren Elemente angeordnet sind, eine kleinere oplische Elasticität(resp. grösseren Brechungsexponenten) und grössere Lichtabsorption zeigen; so z. B. die der Prismennormalen oder die Schwingungsrichtung des ordinären Strahles an den optisch negativen Biotit und Tur- 
malin, und ebenso am Pyroxen, dessen kleinere Elasticitälsaxe bei zunehmendem Eisengehalt sich der Richtung der Prismen- und Pinakoidnormalen nähert. Im Gegensatze hierzu haben die dem Olivin sich anschliessenden optisch positiven rhomboëdrischen Silicate die metallischen Elemente den Endflächen der rhomboëdrischen Molekularcomplexe entsprechend angeordnet, während die Si-Atome an die Seiten gestellt sind.

Die optischen Anomalien lassen sich nach dieser krystallochemischen Theorie auffassen als eine naturliche Folge der mehr oder weniger vollständigen Zergliederung des Grundmoleküls (physikalisches resp. Krystallmolekül) in seine weniger symmetrischen Partialmoleküle (chemische Molekule), beruhend auf einer. durch Druck oder Temperaturabnahme verursachten, im Allgemeinen geringen Veränderung in der gegenseiligen Lage der Elementaratome von einer mebr symmetrischen zu einer weniger symmetrischen schiefen Stellung. Auch sind im Allgemeinen die Anomalien um so grösser, je complicirter ihre Molekule sind, so dass die mehr symmetrischen, besonders die regulären Krystalle eine grössere Anomalie als die weniger symmetrischen zeigen.

Dass diese krystallochemische Theorie nicht nur auf die Silicate, sondern auch auf die ubrigen Mineralien angewendet werden kann, zeigen mehrere Verhältnisse. So z. B. kann man aus der Aehnlichkeit der pyramidalen Winkel, der tetragonalen und der Domen- resp. Prismenwinkel $\left(121^{\circ}-123^{\circ}\right)$ der rhombischen resp. monoklinen isomorphen Oxyde und Salze von der allgemeinen Formel $\mathrm{RO}_{2}$ (Rutil, Cassiterit, Tapiolit, Brookit, Tantalit) schliessen, dass das Grundmolekül der tetragonalen Formen aus quasi-rhombischen Partialformen bestehe, ähnlich den Molekularformen der isomeren oder polymeren rbombischen Mineralien. Ebenso deutet die Aehnlichkeit der Pyramiden winkel $\left(8^{\circ}-81^{\circ}\right)$ des tetragonalen pyramidalhemiëdrischen Scheelits (und des damit isomorphen Reinits), wie des Apatits $\left(3 R_{3} \mathrm{Ca}_{2} \mathrm{O}_{8}+R \mathrm{Rl}_{2}\right)$ und seiner isomorphen Verwandten auf eine zwillingsartige Zusammensetzung aus vier resp. sechs monosymmetrischen Partialformen, ähnlich den Zwillings- resp. Drillingscomplexen von Wolframit mit (011) resp. (023) als Zwillingsebenen, wodurch die Hemiëdrie und optische Anomalie des Scheelits und Apatits erklärt werden. Weiter zeigt die Aehnlichkeit der Rhomboëderwinkel (1050—1070) der rhomboëdrischen Carbonate mil dem Domen- resp. Pyramidenwinkel der rhombischen und monoklinen, dass die mehr symmetrischen, rhomboëdrischen und rhombischen Molekularformen aus drei resp. zwei Partialformen (oder Multiplen davon) bestehen, welche der Molekularform des monoklinen Barytocalcits analog sind, und dass sie also wie die vorher genannten in einem polymeren Molekurverbältnisse zu einander stehen, was für Calcit und Aragonit schon fruher von Tschermak (Lehrbuch der Mineralogie) angenommen worden ist. 
Die oben gemachten Zusammenstellungen zwischen den theoretischen und empirischen chemischen Daten stimmen wohl im Allgemeinen nicht so genau mit einander überein, wie einige andere dergleichen Zusammenstellungen, so z. B. die von Tschermak für die Glimmer- und Ghloritgruppen. Dieses liegt jedoch in der Natur der Sache. Wenn man, wie ich hier, eine neue Richtung einscblägt, muss man, von dem Allgemeinen zum Einzelnen gehend, sich im Anfange mit approximativen Resultaten begnügen.

Was diese Richtung oder die Verbindung der krystallographischen und chemischen Forschungsresultate betrifft, so ist ihre Berechtigung wohl einem Jeden so einleuchtend, dass eine weitere Vertheidigung nicht vonnöthen sein möchte. Die einseitige chemische Untersuchung der Mineralien ist so weit geführt, dass weniges mehr allein auf diesem Wege für die Kenntniss der Constitution der Mineralien zu hoffen ist. Da man in der organischen Chemie sich nicht mehr mit den bisher angewendeten Structurformeln begnügt, sondern stereochemische Formeln zu construiren versucht, so ist ein solcher Versuch in der Mineralogie noch mehr an seinem Platze, da man in dieser im Allgemeinen mehr Rücksicht auf die Formenverhältnisse nimmt als in der Chemie.

Schliesslich gebe ich hier noch eine Uebersicht des krystallochemischen Systems der Silicate, welches ich in oben angeführter Abhandlung aufgestellt babe. Dieses gründet sich wohl zunächst auf meine krystallochemische Theorie, aber ich habe hier ausser den chemischen und krystallographischen auch die physikalischen Eigenschaften der Mineralien berúcksichtigt, besonders den wichtigen Unterschied zwischen metallischen und nichtmetallischen Mineralien, um ein möglichst natürliches System der Mineralien zu erhalten, mich hierbei in der Hauptsache den von $\mathrm{Nau} \mathrm{mann}$ in den alteren, von ibmó selbst redigirten Auflagen seiner Mineralogie aufgestellten Principien der mineralogischen Systematik anschliessend.

\section{Krystallochemisches System der Silicate.}

\section{Classe I. Geolithe.}

1. Plagioklas (Anorthit, Andesin, Albit). - Mikroklin. Orthoklas. - Spodumen. Petalit. Wollastonit. - Danburit. Leukophan. Melinophan.

2. Skapolith (Gehlenit, Sarkolith). - Nephelin (Cancrinit, Davyn). Milarit.

3. Leucit. - Sodalith (Nosean, Hauyn).

\section{Classe II. Hydrogeolithe (Zeolithe).}

1. Datolith. Prehnit. - Desmin (Harmotom, Phillipsit). Heulandit (Brewsterit, Epistilbit). - Skolezit. Natrolith (Mesolith). Thomsonit. - Laumontit (Leonhardit). - Okenit (Pektolith). 
2. Apophyllit. - Chabasit (Gmelinit, Levyn, Herschelit).

3. Analcim. Faujasit. Pollux.

Classe III. Phyllithe.

1. Muscovit (Paragonit, Margarit, Lepidolith). Biotit (Meroxen, Phlogopit, Zinnwaldit). Glintonit. - Klinochlor (Pennin). - Talk. Serpentin. - Pyrophyllit. Kaolin.

2. Biotit (z. Th.). Prochlorit.

Classe IV. Amphoterolithe.

1. Axinit. - Epidot (Piemontit). Orthit (Mosandrit). Karpholith (Ardennit). Zoisit (Thulit). - Gadolinit (Homilit). Euklas. - Gordierit (Pinit).

2. Turmalin. Cappelenit (Melanocerit, Karyocerit, Tritomit). Eudialyt. Idokras (Melilith).

3. Granat. Helvin.

Classe V. Stylolithe.

1. Sapphirin. Cyanit. Dumortierit. Andalusit (Sillimanit, Xenolith). Staurolith. - Topas. Bertrandit.

2. Beryll. - Thorit (Orangit). Zirkon (Malakon).

3. Zunyit.

\section{Classe VI. Metallolithe.}

1. Rhodonit. Babingtonit. Pyroxen (Malakolith, Diallag, Diopsid, Augii). Akmit (Aegirin). Wöhlerit. - Amphibol (Tremolit, Grammatit, Amphibol-Anthophyllit, Hornblende). Arfvedsonit. - Enstatit (Bronzit, Hypers(hen). Anthophyllit (Gedrit). Astrophyllit. - Titanit (Yttrotitanit). Guarinit. Liëvrit. - Olivin (Forsterit, Hyalosiderit, Fayalit, Tephroit). Monticellit. Chondrodit (Humit, Klinohumit). - Calamin. Cerit.

2. Willemit (Troostit). Phenakit. Dioptas. - Pyrosmalith.

3. Eulytin. 